\title{
Synthesis and configurational assignment of vinyl sulfoximines and sulfonimidamides
}

\author{
Gregory B. Craven, ${ }^{\dagger,}, *$ Edward L. Briggs, ${ }^{\dagger}$ Charlotte M. Zammit, ${ }^{\dagger}$ Alex McDermott, ${ }^{\dagger}$ Stephanie \\ Greed, ${ }^{\dagger}$ Dominic P. Affron, ${ }^{\dagger}$ Charlotte Leinfellner, ${ }^{\dagger}$ Hannah Cudmore, ${ }^{\dagger}$ Ruth R. Tweedy, ${ }^{\dagger}$ Renzo \\ Luisi,§ James A. Bullt,* and Alan Armstrongt,*
}

† Department of Chemistry, Imperial College London, Molecular Sciences Research Hub, White City Campus, 80 Wood

Lane, London W12 0BZ, U.K.

₹ School of Molecular and Cellular Biology, The Astbury Centre for Structural Molecular Biology, University of Leeds, LS2 9JT, UK.

§ Department of Pharmacy - Drug Sciences, University of Bari, “A. Moro” Via E. Orabona 4, Bari 70125, Italy.

\begin{abstract}
Vinyl sulfones and sulfonamides are valued for their use as electrophilic warheads in covalent protein inhibitors. Conversely, the S(VI) aza-isosteres thereof, vinyl sulfoximines and sulfonimidamides, are far less studied and have yet to be applied to the field of protein bioconjugation. Herein, we report a range of different synthetic methodologies for constructing vinyl sulfoximine and vinyl sulfonimidamide architectures that allows access to new areas of electrophilic chemical space. We demonstrate how late stage functionalization can be applied to these motifs to incorporate alkyne tags, generating fully functionalized probes for future chemical biology applications. Finally, we establish a workflow for determining the absolute configuration of enantioenriched vinyl sulfoximines and sulfonimidamides by comparing experimentally and computationally determined electronic circular dichroism spectra, enabling access to configurationally assigned enantiomeric pairs by separation.
\end{abstract}

\section{INTRODUCTION}

Sulfoximines and sulfonimidamides, the mono aza-analogues of sulfones and sulfonamides respectively, are gaining traction as valuable pharmacophores in medicinal chemistry (Figure 1a).1,2 Architecturally, these motifs carry asymmetry at the tetrahedral sulfur center, offer hydrogen-bond acceptor and donor functionalities for $\mathrm{NH}$ derivatives, and afford new vectors along which to generate structural complexity. Bioactive sulfoximines date back to the discovery of methionine sulfoximine, which was later discovered to be a prodrug inhibitor of glutamine synthetase. ${ }^{3}$ More recently, new synthetic methodologies have facilitated the incorporation of sulfoximine and sulfonimidamide motifs into drug discovery programs, through which their high chemical and stereochemical stability as well as favourable physicochemical properties have been uncovered. ${ }^{4} \mathrm{In}$ deed, three sulfoximine compounds have entered clinical trials in recent years, with their desirable DMPK profiles being cited as key triggers for candidate selection. ${ }^{5}$

Vinyl sulfones and sulfonamides are well characterized electrophiles and have been extensively utilized as reactive warheads in protein-targeted covalent probes. ${ }^{6}$ For example, substituted vinyl sulfones are found in cysteine-targeting covalent cathepsin inhibitors, 7 terminal vinyl sulfones have been used in lysine-targeting cyclin-dependent kinase 2 (Cdk2) inhibitors ${ }^{8}$ and terminal vinyl sulfonamides have been employed to target cysteine residues on a variety of proteins including ERK2,9 KRas(G12C), ${ }^{10}$ BTK 11 and USP712 (Figure 1b). Synthetic routes to these scaffolds are well established and probe specificity is engineered by installation of pharmacophores that bind the target protein and orientate the electrophile to react with a nearby nucleophilic amino acid. ${ }^{13}$ 
a) $\mathrm{S}(\mathrm{VI})$ aza-isosteres
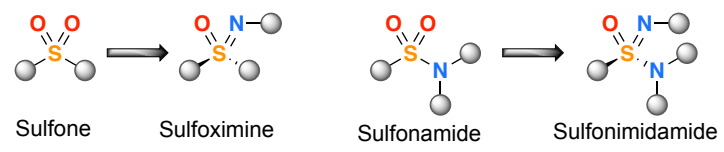

b) S(VI) Covalent Probes

Substituted vinyl sulfones

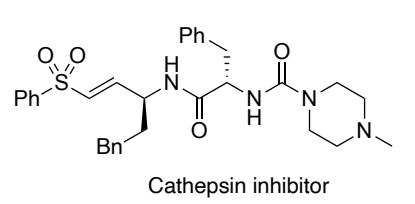

Terminal vinyl sulfones
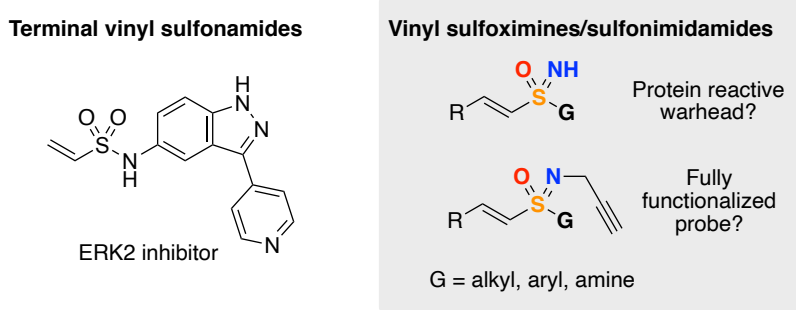

Figure 1. a) S(VI) aza-isosteres. b) Examples of established vinyl sulfone- and sulfonamide-based covalent inhibitors and proposed scaffolds for future aza-analogues.

We speculated that vinyl sulfoximines and sulfonimidamides could similarly act as protein reactive warheads in covalent probes. ${ }^{14}$ The value of these aza-analogues compared to the established vinyl S(VI) electrophiles would arise from their ability to offer alternative hydrogen bonding architectures, additional vectors for pharmacophore incorporation and likely different reactivity profiles. Moreover, the new nitrogen center offers a convenient handle for the attachment of chemical biology functionalities, such as alkynes and fluorophores, to generate fully functionalized probes. ${ }^{15}$ As these derivatives are chiral at sulfur, they present increased complexity and potential complementarity as probes. Recent work from Cravatt has demonstrated the value of using stereochemically-paired fully functionalised probes in the context of proteomic screening, offering an internal control for non-specific protein binding. ${ }^{16}$ More broadly, electrophilic fragments are now routinely screened to identify starting points for covalent probes and novel electrophilic functionalities are desirable to expand the diversity of covalent fragment libraries. ${ }^{17,18}$

Over the last 30 years, $\beta$-substituted $E$-vinyl sulfoximines have been prepared by a variety of approaches utilizing carbonyl addition then $\beta$-hydroxy activation/elimination, ${ }^{19}$ Peterson olefination ${ }^{20}$ and Horner-Wadsworth-Emmons (HWE) olefination $^{21}$ (Scheme 1). More recently, Arvidsson reported an improved HWE sequence that is both high yielding and protecting group free. ${ }^{22}$ These methodologies demonstrate the chemical stability and tractability of the vinyl sulfoximine motif and one such recent study has given rise to the first examples of bioactive vinyl sulfoximines that are thought to activate the transcription factor Nrf2, although a covalent mechanism of action was not proposed. ${ }^{23}$ However, these synthetic routes have clear limitations; they generally utilize multiple-step sequences that are reliant on the use of hydrazoic acid or $O$-(mesitylenesulfonyl)hydroxylamine MSH for sulfoxide imidation, which are both known for their thermal instability.24,25 There are very few examples of terminal vinyl sulfoximines or enantioenriched vinyl sulfoximines. Moreover, the first two examples of vinyl sulfonimidamides were only reported very recently, ${ }^{26}$ despite the prevalence of the analogous vinyl sulfonamides, which we attribute to the historically limited synthetic methodology regarding sulfonimidamides. 
Scheme 1. Previous routes to vinyl sulfoximines.

Substituted $E$-vinyl sulfoximines via $\beta$-hydroxy elimination

$$
\begin{aligned}
& \text { Protection } \\
& \text { Hwang (1987): imidation with } \mathrm{NaN}_{3} / \mathrm{H}_{2} \mathrm{SO}_{4} \text {; elimination with } \mathrm{CDI} \\
& \text { Craig (1993): imidation with } \mathrm{NaN}_{3} / \mathrm{H}_{2} \mathrm{SO}_{4} \text {; elimination with } \mathrm{MeOCOCl}
\end{aligned}
$$

Substituted E-vinyl sulfoximines via HWE reaction

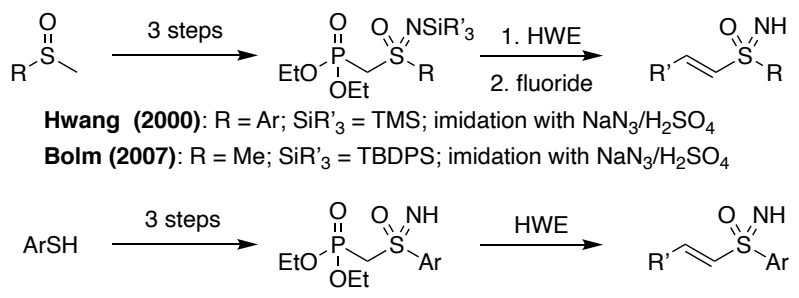

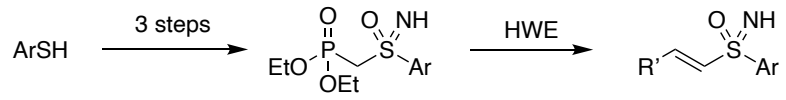

Arvidsson (2016): PG-free; high-yielding; imidation with MSH

It is notable that in recent years the methods for sulfoximine and sulfonimidamide synthesis have developed considerably.27-29 In our groups we have developed a safe and effective method for the NH transfer to sulfoxides to form sulfoximines using ammonium carbamate and (diacetoxy)iodobenzene. ${ }^{30}$ Similarly, we have developed a 1-pot oxidation and imidation protocol for the direct conversion of sulfides to sulfoximines. ${ }^{31}$ Stockman and Lücking established a related process for the preparation of sulfonimidamides from sulfinamides. ${ }^{32}$ We have developed the synthesis of sulfonimidamides from sulfenamides by $\mathrm{NH}$ and 0 transfer. ${ }^{33}$ It is notable that by using this $\mathrm{NH}$ transfer methodology, the reaction of phenylvinylsulfoxide was effective in generating phenyl vinyl sulfoximine, though in moderate yield (54\%). ${ }^{30}$ However, the corresponding method from the sulfide was ineffective (14\% yield for phenylvinyl sulfide), ${ }^{31 a}$ compounded by difficulties in the synthesis and acid instability of terminal vinyl sulfides. Improved methods that expand the diversity of accessible products, minimise the use of dangerous reagents and enable the synthesis of fully functionalised probes for future chemical biology investigations would be of considerable interest.

Here we report the development of new and improved synthetic routes for the preparation of vinyl sulfoximines, exploiting recent developments in the synthesis of aza-S(VI) derivatives. Three orthogonal routes to access terminal and substituted vinyl $\mathrm{NH}$-sulfoximines are investigated, including highly enantioenriched examples (Scheme 2). Furthermore, we report some of the first examples of vinyl $\mathrm{NH}$-sulfonimidamides. These studies considerably extend the scope of the hypervalent iodine mediated NH-transfer methods and provide a comparison of available routes, highlighting strategies for different substitution patterns. A simple propargylation reaction to convert these products into fully functionalised probes is demonstrated. Finally, we generate stereochemically-paired vinyl sulfoximine and sulfonimidamide derivatives, by late-stage chiral separation, and establish a method for their configurational assignment by combining DFT calculations and circular dichroism. These approaches allow access to a range of stereochemically defined structures of interest as probes.

Scheme 2. Synthetic methods described in this work to access vinyl sulfoximines and sulfonimidamides.

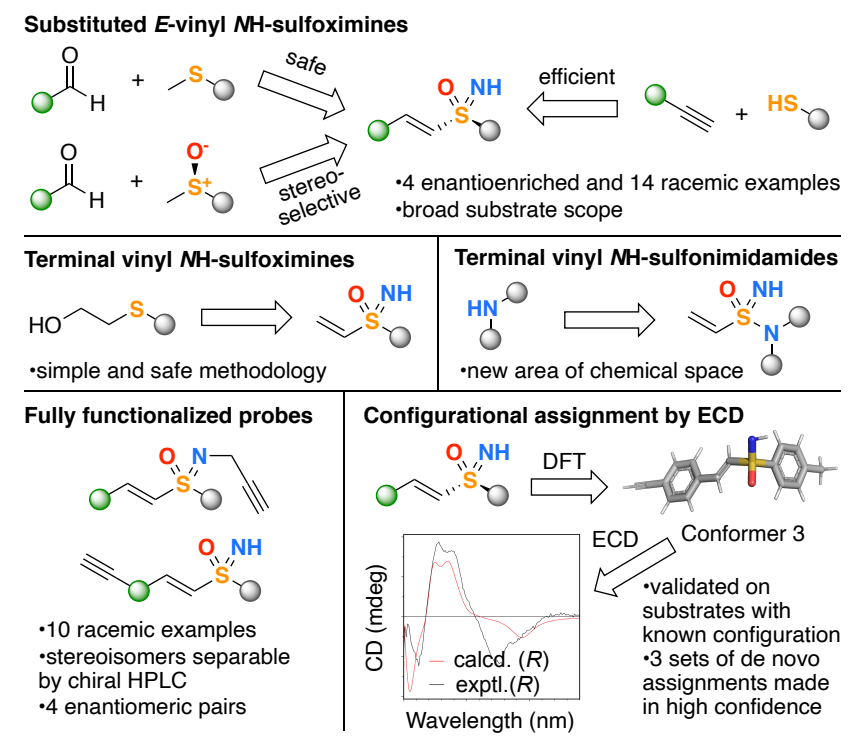




\section{RESULTS AND DISCUSSION}

Substituted vinyl $E$-sulfoximines from methyl sulfides and sulfoxides. Our initial investigations focussed on the established HWE approach to vinyl sulfoximine synthesis but commencing from simple sulfides and applying recent methods for $\mathrm{NH}$ transfer, using (diacetoxy)iodobenzene and ammonium carbamate, to improve the efficiency and safety. ${ }^{31 a}$ Accordingly, methyl sulfides $\mathbf{1 a}$ and $\mathbf{1 b}$ were converted into the corresponding $N \mathrm{H}$-sulfoximines $\mathbf{2 a}$ and $\mathbf{2 b}$ in very good yields (Scheme 3a). After TBDPS-protection of the sulfoximine nitrogen to give intermediates $\mathbf{3 a}$ and $\mathbf{3 b}$, a one-pot HWE procedure was implemented using a range of aldehyde coupling partners to furnish $N$ TBDPS-protected vinyl sulfoximines $\mathbf{4 a - 4 g . ~ F i n a l l y , ~}$ TBDPS-deprotection with TBAF afforded vinyl $\mathrm{NH}$-sulfoximines $\mathbf{5 a - 5 g}$. Both aromatic and aliphatic aldehydes gave similarly high yields for the two steps (5a and $\mathbf{5 b}$ ). Incorporation of an alkyne functionality was well tolerated (5c and $\mathbf{5 e}$ ), which is encouraging for the potential to generate future fully functionalised probes. The method also performed well using the more electron rich anisole-derived sulfoximine (5e-5g) and using heteroaromatic aldehydes (5d, 5f, 5g).

Scheme 3. Synthesis of substituted $E$-vinyl sulfoximines from methyl sulfides and sulfoxides.

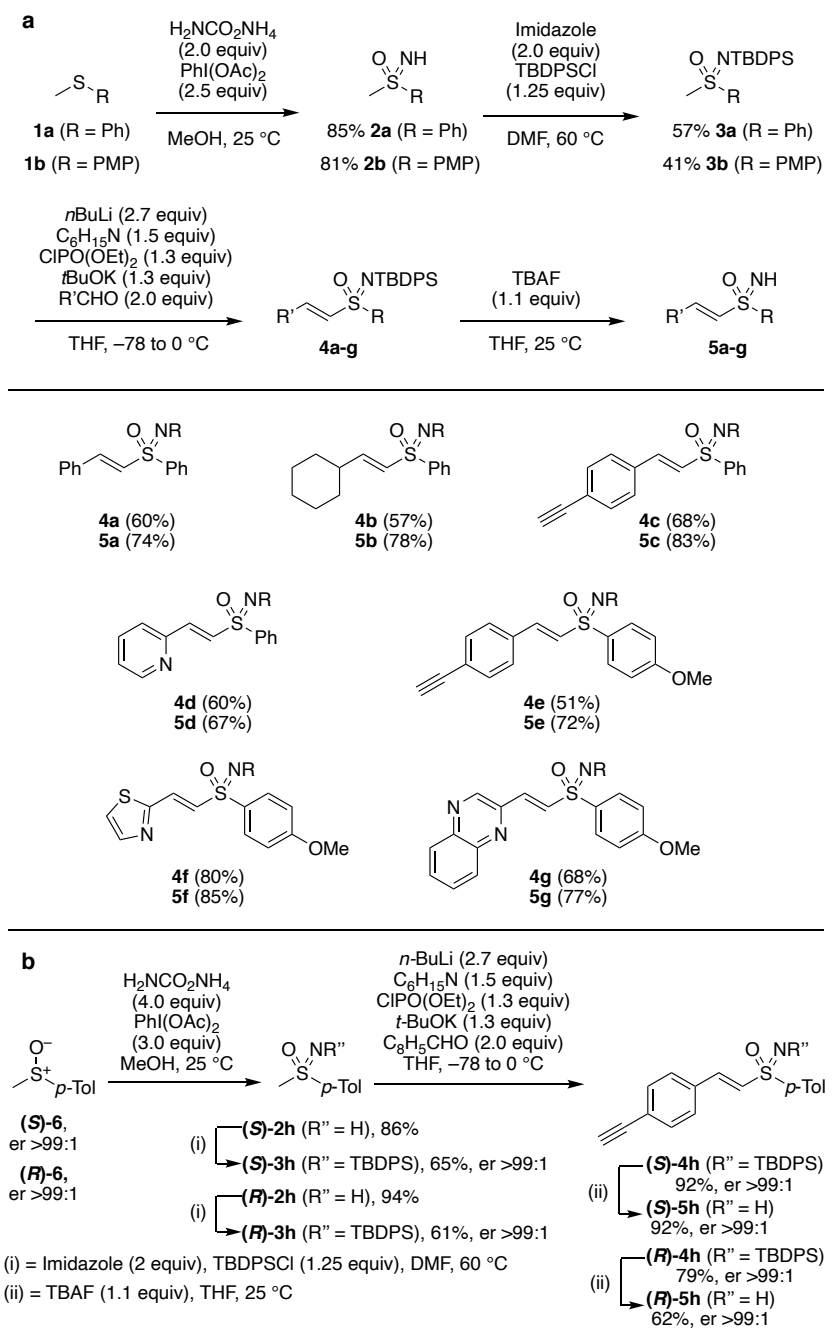

Stereochemically enriched sulfoximines are finding increasing utility in drug discovery and asymmetric synthesis but represent synthetically challenging targets. ${ }^{4,5,34}$ Application of an analogous synthetic sequence to enantioenriched sulfoxides $(S)-6$ and $(R)-6$ using 4-ethynylbenzaldehyde furnished products $(S)-5 h$ and $(R)-5 h$ respectively, with retention of the configuration at sulfur and without loss of enantiomeric excess (Scheme $3 \mathrm{~b}$ ). ${ }^{30}$ The stereospecificity of this route and configurational stability of the intermediates and products is encouraging for the design of future stereochemically-defined vinyl sulfoximines. Indeed, powerful methods are well established for the enantioselective oxidation of unsymmetrical methyl sulfides, ${ }^{35}$ which could be combined with this HWE route to provide access to a broad range of enantioenriched vinyl sulfoximines. By incorporating alkyne functionalities into $(\boldsymbol{S})-\mathbf{5 h}$ and $(\boldsymbol{R})-\mathbf{5 h}$, these structures represent the first example of stereochemically-paired fully functionalised vinyl sulfoximine probes.

Substituted $E$-vinyl sulfoximines via alkyne hydrothiolation. Next, we sought to develop a more efficient route to vinyl sulfoximines to expand the product scope, and notably avoid the use of protecting groups and possible regiochemical ambiguity in the deprotonation step of dialkyl sulfoximines. We anticipated that direct oxidation/imidation of vinyl thioethers 
would be the most direct path. A range of metal-catalysed alkyne hydrothiolation reactions have been developed that facilitate the synthesis of either branched, 36,37 linear $E$-vinyl thioethers ${ }^{38}$ or linear Z-vinyl thioethers ${ }^{39}$ in high selectivity. For this study we were interested in linear $E$-vinyl sulfoximines and applied a Wilkinson's catalyst mediated alkyne thiolalkyation methodology reported by Love which gives high levels of stereocontrol using 1,2-dichloroethane (DCE) as solvent.40 The $E$-vinyl thioether products $\mathbf{9}$ were then converted to the corresponding $E$-vinyl sulfoximines $\mathbf{1 0}$ using our NH/O transfer method (Scheme 4).31a This two-step sequence provided efficient access to a diverse range of vinyl sulfoximines in good to excellent yields, tolerating silyl (10a-10b), aryl (10c-10e) and alkyl (10f) alkynes in combination with aryl (10a, 10c, 10d and 10f) and alkyl (10b and 10e) thiols. The success of this methodology for alkyl thiols is particularly exciting, since access to such products by HWE chemistry is challenging. Indeed, the incorporation of an aryl bromide containing alkyne (10e) and thiol (10d) will enable elaboration of these scaffolds in multiple vectors using transition metal catalyzed coupling chemistry. Pleasingly, we were also able to Boc-deprotect $10 \mathrm{f}$ to afford amine $10 \mathrm{~g}$, offering a useful handle for functionalization at the amine.

\section{Scheme 4. Synthesis of substituted $E$-vinyl sulfoximines via alkyne hydrothiolation.}

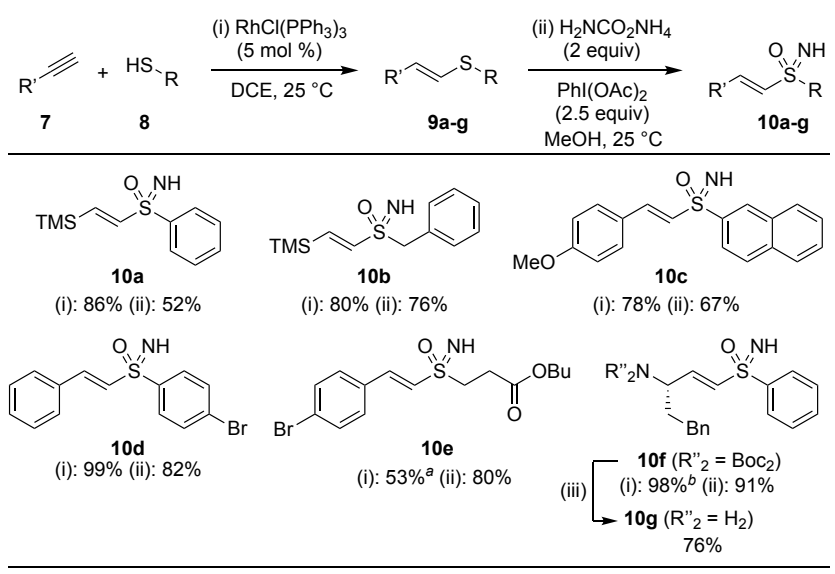

a Used $10 \mathrm{~mol} \% \mathrm{RhCl}\left(\mathrm{PPh}_{3}\right)_{3}, 65^{\circ} \mathrm{C}$. ${ }^{b} 9 \mathrm{f}$ is exemplified in ref $13 \mathrm{e}$ using similar conditions

Terminal vinyl sulfoximines via alcohol elimination. An alternative synthetic approach was needed to access terminal vinyl sulfoximines. We implemented a two-step sequence based on the controlled activation and elimination of $\beta$-hydroxy ethyl sulfoximines (Table 1). $\beta$-Hydroxy thioethers 11a-d were converted to the corresponding sulfoximines 12a-d in fairto-excellent yields. Treating alcohols 12a-d with a slight excess of methanesulfonyl chloride and base led to activation and elimination of the alcohol to give the desired NH vinyl sulfoximines 13a-d. The methodology is suitable for producing aromatic (13a and 13b), heteroaromatic (13c) and alkyl (13d) vinyl sulfoximines. The mesylation step generally proceeded with good $O / N$ chemoselectivity; however, we were additionally able to isolate the $N$-mesyl vinyl sulfoximines in some cases (14a and 14d) as side products. We anticipate that the $N$-mesyl vinyl sulfoximines would be more electrophilic and reactive than the $\mathrm{NH}$ analogues and may represent an interesting new class of electrophile in their own right.

Table 1. Synthesis of terminal vinyl sulfoximines via alcohol elimination.

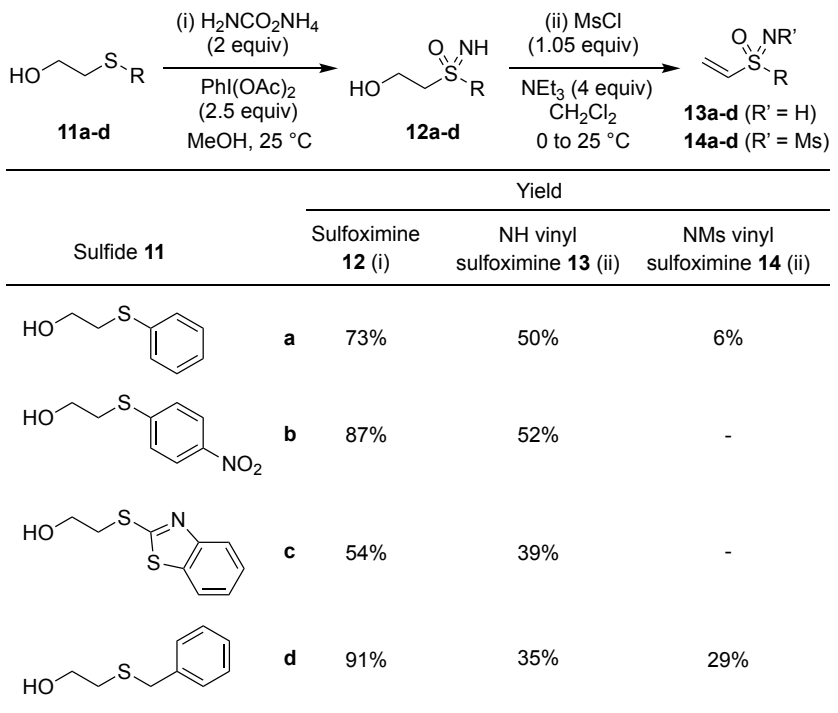

Terminal vinyl sulfonimidamides via alcohol elimination. We envisioned that an analogous $\beta$-hydroxy ethyl activation/elimination strategy could be implemented to generate vinyl sulfonimidamides by combining it with our sulfenamide oxidation/imidation method. ${ }^{33}$ Accordingly, phenyl piperidine (15a) was treated with THP-protected $\beta$-hydroxy ethyl disulfide (16) and silver nitrate to generate the corresponding sulfenamide (Scheme 5). The inclusion of Et ${ }_{3} \mathrm{~N}$, in modified 
conditions, gave improved results by reducing the acidity of the reaction. The sulfenamide was then simultaneously imidated and oxidized using ammonium carbamate and iodosylbenzene to give the THP-protected $\beta$-hydroxy sulfonimidamide 17a in $57 \%$ yield over two steps. After THP-deprotection, mesylation and in situ elimination afforded the desired vinyl sulfonimidamide 18a in $46 \%$ over two steps. Notably, the mesylation reaction proceeded with excellent $O / N$ chemoselectivity for the alcohol over the sulfonimidamide. This novel class of electrophile was stable towards silica gel chromatography and has shown no noticeable degradation when stored at $-20^{\circ} \mathrm{C}$ in DMSO over six months. To further exemplify the methodology, we synthesised three additional vinyl sulfonimidamides 18b-18d. Starting with racemic 1-methyl-3-phenylpiperazine 16b gave vinyl sulfonimidamide 18b (d.r. 1:2). Encouragingly the reaction sequence was successful with nortriptyline 15c, demonstrating that bioactive motifs can also be converted into vinyl sulfonimidamides. This route has delivered some of the first examples of a chemical motif that has significant potential for future probe design.

Scheme 5. Synthesis of terminal vinyl sulfonimidamides via alcohol elimination.

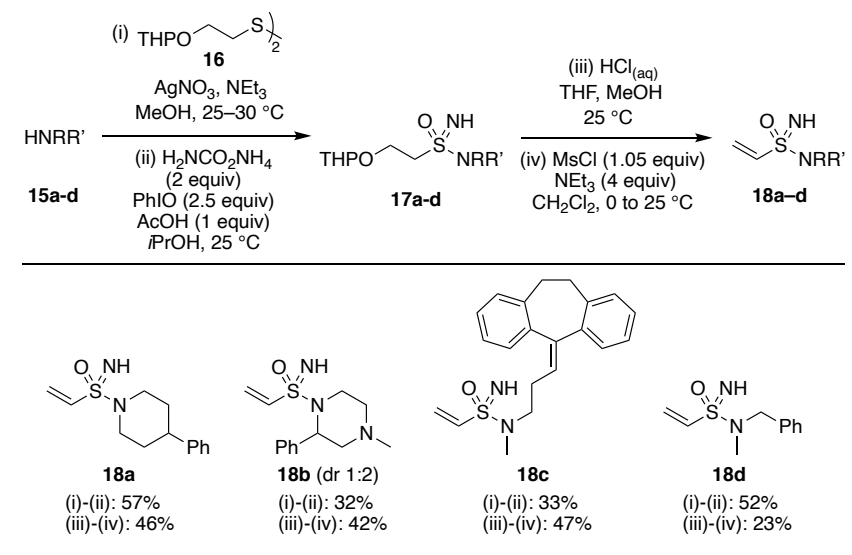

$\boldsymbol{N}$-Propargylation of vinyl sulfoximines and sulfonimidamides. The incorporation of alkyne tags onto small molecules is a highly valuable transformation that enables chemical biology experiments such as activity-based protein profiling and fluorescence microscopy. ${ }^{41}$ In the context of covalent protein targeting probes, the addition of an alkyne tag generates a fully functionalized probe that facilitates visualization and quantification of target engagement. ${ }^{15}$ The majority of the previously established covalent warheads do not possess convenient chemical handles for attachment of alkyne tags. ${ }^{14 \mathrm{~b}}$ As such the design of fully functionalised probes often requires preinstallation of the alkyne handle into the pharmacophore which can be synthetically challenging. ${ }^{42}$ Therefore, a general method for late-stage propargylation of small molecules would present significant benefits and opportunities to chemical biologists.

Functionalization, including propargylation, of sulfoximine- $N H$ groups has been widely reported, though for sulfonimidamides much less so. ${ }^{4-45}$ Vinyl sulfoximines and sulfonimidamides are electrophilic in nature which raises potential reaction compatibility concerns. Pleasingly, however, a diverse range of vinyl sulfoximines and sulfonimidamides could be efficiently propargylated using sodium hydride and propargyl bromide (Scheme 6). Terminal (19a), substituted (19b and 19c) and heteroaromatic (19d) vinyl sulfoximines as well as tertiary amine containing vinyl sulfonimidamides (19e,f) and bioactivepharmacophore containing vinyl sulfonimidamide $(\mathbf{1 9 g})$ were all well tolerated.

Scheme 6. $N$-Propargylation of vinyl sulfoximines and sulfonimidamides.

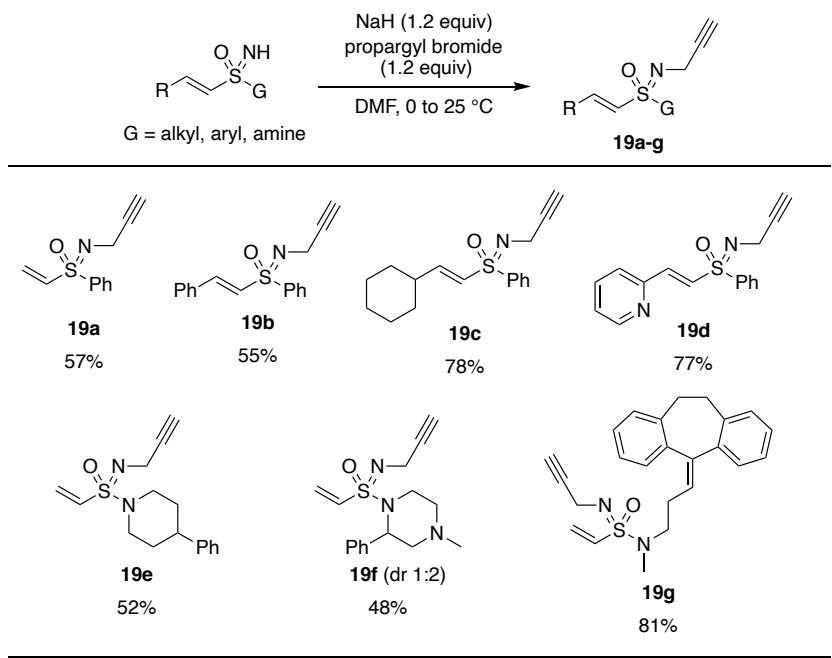


Configurational assignment of enantioenriched vinyl sulfoximines and sulfonimidamides. The binding between proteins and chemical probes often exhibits discriminatory affinity for different stereoisomers. Indeed, the clinical candidate sulfoximine inhibitors roniciclib, atuveciclib and ceralasertib are all enantiopure. ${ }^{5}$ Therefore, the development of future probes relies on both access to enantioenriched vinyl sulfoximines and sulfonimidamides and the ability to assign their absolute configuration. Through the HWE reaction sequence, highly enantioenriched vinyl sulfoximines $(\boldsymbol{S})-\mathbf{5 h}$ and $(\boldsymbol{R})-\mathbf{5 h}$ (Scheme $3 b$ ) were prepared with synthetically defined configuration. However, the other synthetic routes to vinyl sulfoximines and sulfonimidamides described herein generate racemic products with respect to the chiral sulfur centre. Chiral separation, typically conducted by HPLC or SFC, offers an alternative method for accessing enantioenriched products and has been highly successful with regards to the production of enantiopure sulfoxide drugs. ${ }^{46,47}$ The value of such separation is dependent on reliable assignment of absolute configuration which is typically accomplished by X-ray crystallography, ${ }^{48} \mathrm{NMR}^{49}$ or computational and experimental circular dichroism. ${ }^{50}$ Electronic circular dichroism (ECD) is among the most versatile of these techniques and has previously been successfully applied to the stereochemical assignment of sulfoxide drugs and sulfonimidamides.51-53 To establish whether ECD could be reliably applied to the configurational assignment of vinyl sulfoximines and sulfonimidamides we first investigated whether quantum mechanical calculations could predict the experimentally observed ECD spectrum for $(\boldsymbol{R})$-5h with high confidence.

A conformational search (see Supporting Information) was performed on the vinyl sulfoximine unit using the B3LYP functional and the $6-31 \mathrm{G}^{*}$ basis set. The low energy conformers (within $3 \mathrm{kcal} / \mathrm{mol}$ of the lowest) were further optimised using cam-B3LYP/6-311++g(d,p) in a conductor-like polarizable continuum (CPCM) model of acetonitrile. We identified four minimum energy conformers of $(\boldsymbol{R})-\mathbf{5 h}$ that are significantly populated at ambient temperature (Figure 2a). In the two lowest energy conformers ( 3 and 1 ), the vinyl group eclipses the $S=0$ bond, with the two structures varying in the orientation of the NH group. Conformers 15 and 13, which are slightly higher in energy, have the vinyl group eclipsing the $\mathrm{S}=\mathrm{N}$ bond. The electronic transitions of each conformer of $(\boldsymbol{R})-\mathbf{5 h}$ were then calculated and the UV-vis and ECD spectra simulated and summed according to their Boltzmann weighting (Figure 2b). It should be noted that the calculated ECD spectra of conformers 3 and 1 were similar to each other in the experimental range (190-340 nm) whilst the calculated spectra of conformers 13 and 15 were also similar to each other, but with the significant peaks being opposite in sign to those of $3 / 1$. The weighted ECD spectrum is therefore sensitive to the relative conformer energies at the vinyl-S=0 dihedral. We then measured the ECD and UVvis spectra of $(\boldsymbol{R})-\mathbf{5 h}$ in acetonitrile experimentally and compared them to the calculated spectra using SpecDis, a dedicated software package for comparing ECD/UV spectral similarity. ${ }^{54}$ Aligning the UV-vis spectra required a $11 \mathrm{~nm}$ positive shift correction factor of the calculated spectrum, giving good spectral matches that are characterized by a sharp peak at $197 \mathrm{~nm}$ and a broad peak between $270-320 \mathrm{~nm}$. Applying the same $11 \mathrm{~nm}$ correction factor to the calculated ECD spectrum gave excellent correlation with the experimental ECD, defined by a trough between 200-217 nm, a double peak between 217-256 $\mathrm{nm}$ and a second trough between 256-316 $\mathrm{nm}$. To quantify the ECD spectral match, we applied similarity factor analysis which gave an $80.7 \%$ match for the experimental $(\boldsymbol{R})-5 \mathbf{h}$ ECD spectrum with the calculated $(\boldsymbol{R})-\mathbf{5 h}$ spectrum but only a $0.7 \%$ match with the calculated $(\boldsymbol{S})$-5h spectrum. The resulting delta score of $80.0 \%$ represents a high confidence assignment and validates that this workflow is reliable and should be suitable for assigning the configuration of vinyl sulfoximines and sulfonimidamides with unknown stereochemistry. 

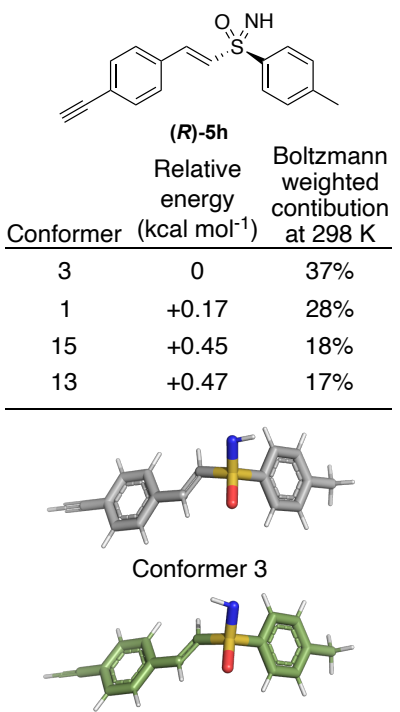

Conformer 1

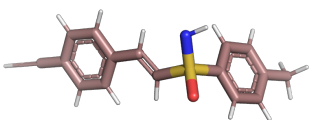

Conformer 15

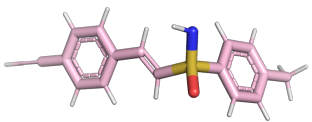

Conformer 13 b)

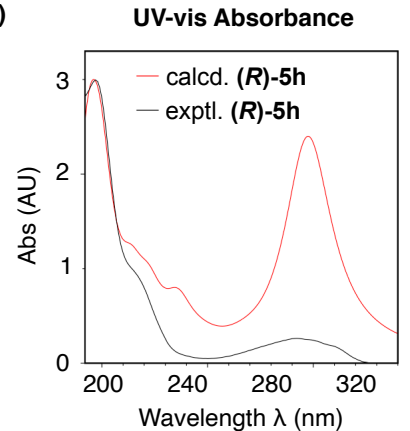

Electronic Circular Dichroism

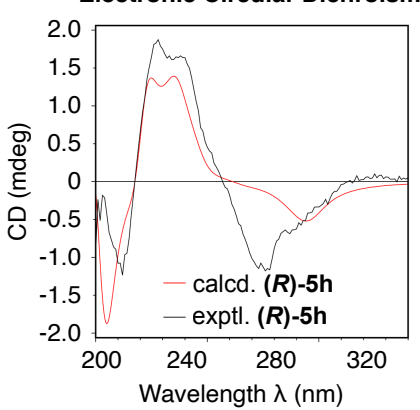

ECD Similarity Factor Analysis Similarity $=80.7 \%$

Enantiomer similarity $=0.7 \%$

Delta score $=80.0 \%$

Figure 2. a) Molecular modelling of the low energy conformations of $(R)-5 h$. b) Comparison of calculated and experimental UV-vis and ECD spectra for $(R)-5 h$.

Next, we selected three compounds $10 \mathrm{~g}, 19 \mathrm{~g}$ and $19 \mathrm{f}$ for chiral separation, which was performed using the automated HPLC/SFC condition screening system at Reach Separations, 55 and de novo configurational assignment by ECD using the same modelling workflow and parameters as described for $(\boldsymbol{R})$-5h. Chiral separation of $\mathbf{1 0 g}$, where the carbon stereocenter is synthetically defined as $(S)$, gave two diastereoisomers $10 \mathrm{~g}-\mathbf{A}$ and $\mathbf{1 0 g}-\mathbf{B}$ as neutral amines. ECD calculation for $\mathbf{1 0 g}$ is challenging because of the presence of the second stereocenter and the conformational flexibility of the acyclic allylic amine. We examined two approaches to simplify this problem. In the first, we used a single fixed, low energy conformation of the vinyl amine portion appended to the four conformers of the allylic sulfoximine unit found for $\mathbf{5 h}$. We modelled both $(\boldsymbol{R}, \boldsymbol{S})-\mathbf{1 0 g}$ and $(\boldsymbol{S}, \boldsymbol{S})$ $\mathbf{1 0 g}$ in this way (Supplementary Figure S1). The $(\boldsymbol{R}, \boldsymbol{S})-\mathbf{1 0 g}$ and $(\boldsymbol{S}, \boldsymbol{S})-\mathbf{1 0 g}$ models gave distinct calculated ECD spectra and comparison with the experimental spectra gave the best match for $10 \mathrm{~g}-\mathrm{A}=(R, S)-10 \mathrm{~g}$ and $10 \mathrm{~g}-\mathrm{B}=(S, S)-10 \mathrm{~g}$, with a mean similarity score of $78.6 \%$ (Supplementary Figure S2). This assignment is made with high confidence given that the mean similarity score for the opposite assignment is only $37.1 \%$. To simplify the modelling further for future applications, we also modelled a phenyl vinyl sulfoximine substructure of $\mathbf{1 0 g}$ that lacks the chiral amine functionality. We found that comparing the predicted ECD spectrum of substructure-10g with the experimental racemic-subtracted ECD spectrum of $\mathbf{1 0 g}$ gave the same assignment as modelling the full structure with a similar confidence level (delta score $=80.6 \%$ ) (Supplementary Figure S3).

Vinyl sulfonimidamide $19 \mathrm{~g}$ was separated into its two enantiomers $19 \mathrm{~g}-\mathbf{A}$ and $\mathbf{1 9 g}-\mathbf{B}$ which were isolated in very high enantiomeric purity. The biaryl system is achiral and was anticipated not to make a significant contribution to the ECD spectrum, while the flexible nature of the alkyl linker would make the conformational modelling challenging. Accordingly, we modelled $(\boldsymbol{R})-\mathbf{2 0}$ as the key substructure which yielded three low energy conformers. In analogy with vinyl sulfoximine $\mathbf{5 h}$ and $10 \mathrm{~g}$, the two lower energy conformers (differing by $0.17 \mathrm{kcal} \mathrm{mol}^{-1}$ ) have the vinyl group eclipsing the $\mathrm{S}=0$ bond, with the two structures varying in the orientation of the $\mathrm{N}$-propargyl group, while for the higher energy conformer $(+0.75 \mathrm{kcal}$ $\mathrm{mol}^{-1}$ ) the vinyl group eclipses the $\mathrm{S}=\mathrm{N}$ bond (Supplementary Figure S4). Comparing the calculated and experimental UV-vis and ECD spectra led to an unambiguous assignment of 19g-A $=(R)$ and 19g-B $=(S)$ configuration with high confidence (delta score $=91.5 \%$ ) (Supplementary Figure S5).

Chiral separation of 19f gave four stereoisomers 19f-A-D that were each isolated in high levels of stereo purity. By comparing the NMR spectra of each isomer, 19f-A and 19f-D were assigned as one enantiomeric pair and 19f-B and 19f-C the other, although no information about their relative configuration could be obtained. We then modelled both $(\boldsymbol{R}, \boldsymbol{S})-\mathbf{1 9 f}$ and $(S, S)$-19f which gave four and five low energy $\left(\leq 1.5 \mathrm{kcal} \mathrm{mol}^{-1}\right)$ conformers respectively, with the piperazine ring adopting a chair-like conformation and the phenyl ring pointing axially in each case (Supplementary Figure S6). Conformers in which the phenyl ring points equatorially were substantially higher in energy $\left(\geq 2.2 \mathrm{kcal} \mathrm{mol}^{-1}\right)$ for both $(\boldsymbol{R}, \boldsymbol{S})$-19f and $(\boldsymbol{S}, \boldsymbol{S})-\mathbf{1 9 f}$ and made little-to-no contribution to the calculated ECD. Of note, while for $(\boldsymbol{R}, \boldsymbol{S})$-19f the two lowest energy conformers have the 
vinyl group eclipsing $S=0$, for $(\boldsymbol{S}, \boldsymbol{S})$-19f the lowest four conformers are of very similar energy, two them having the vinyl group eclipsing $\mathrm{S}=\mathrm{N}$, and two having it eclipse $\mathrm{S}=0$. Determining the configuration of diastereoisomers by ECD is typically challenging because they often have closely related ECD spectral profiles. However, in this case the calculated Boltzmannweighted ECD spectra of all four stereoisomers gave strikingly different shapes. Comparing the UV and ECD spectra of each isomer with the modelled spectra, we found that 19f-A has a nearly exact match $(98.9 \%)$ with $(\boldsymbol{R}, \boldsymbol{S})-\mathbf{1 9 f}$ and $\mathbf{1 9 f}-\mathbf{B}$ gave an excellent match $(97.4 \%)$ with $(\boldsymbol{S , S})$-19f, allowing us to make a high confidence assignment of all four isomers (Supplementary Figure S7).

Table 2. Chiral separation and configurational assignment of vinyl sulfoximine and sulfonimidamides stereoisomers by molecular modelling and ECD.

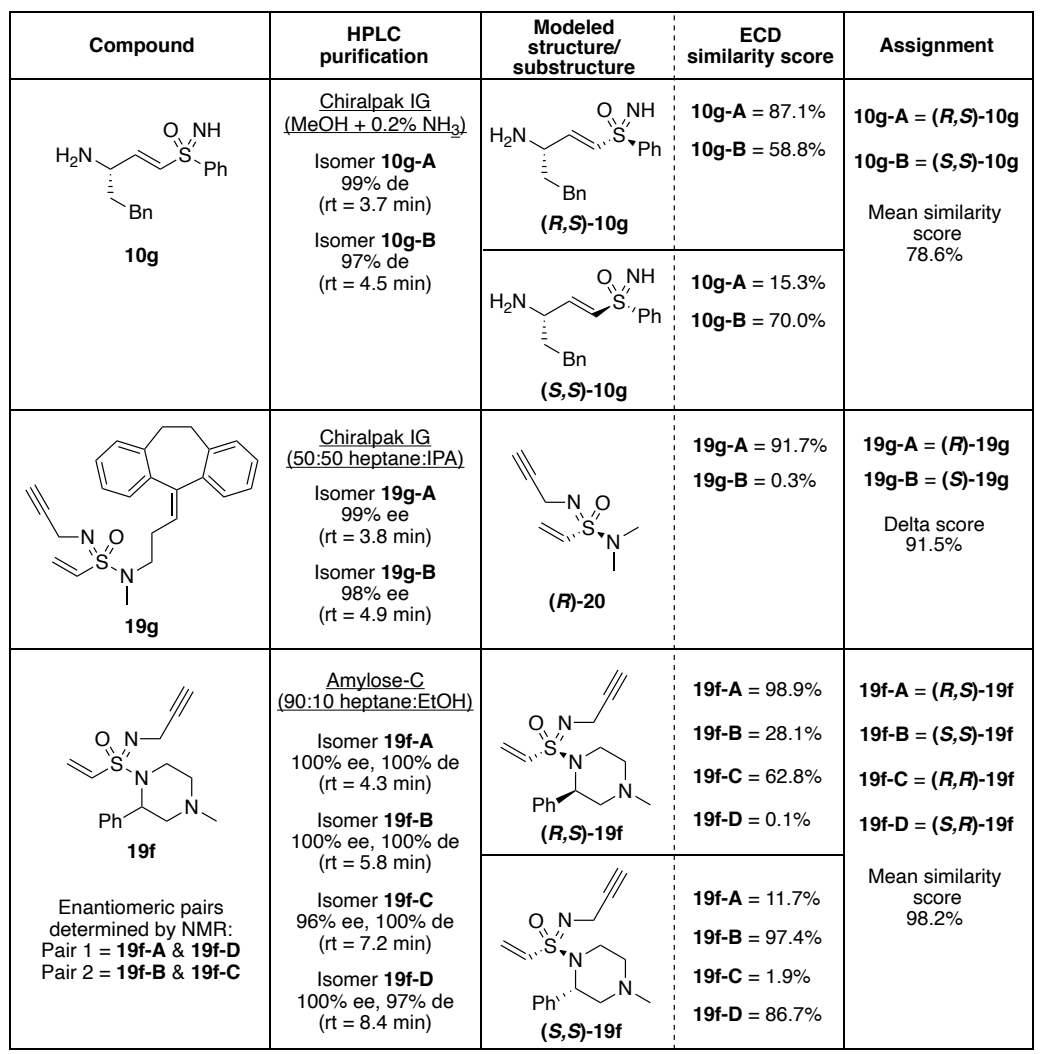
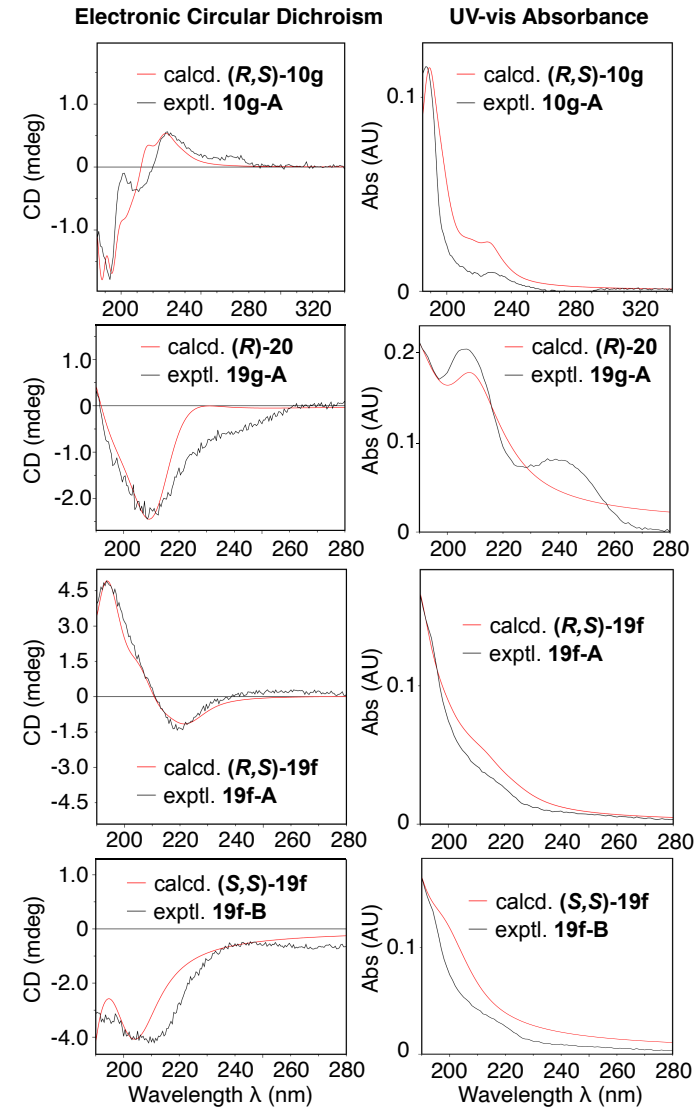

\section{CONCLUSION}

Vinyl sulfoximines and sulfonimidamides offer significant potential as functionalizable electrophilic warheads but have seen little exploration to date. We have developed several new synthetic approaches to these motifs that expand the potential of these motifs and access a much wider range of derivatives. Firstly, we showcased a modified HWE sequence, from methyl sulfides, providing NH vinyl sulfoximines exclusively as the $E$-isomers. Application of this HWE sequence to enantioenriched methyl sulfoxides gave an efficient route to enantioenriched vinyl sulfoximines. Next, we developed a two-step route to vinyl sulfoximines that exploits alkyne hydrothiolation and a highly chemoselective one-pot transfer of $\mathrm{NH}$ and $\mathrm{O}$ to vinyl sulfides. This route is substantially shorter than previous approaches and works well for both alkyl and aryl vinyl sulfoximines.

Terminal vinyl sulfoximine and sulfonimidamides represent particularly challenging synthetic targets that could have widespread application as warheads in covalent probes. We developed a simple two-step route to terminal vinyl sulfoximines from $\beta$-hydroxyethyl thioethers involving sulfoximine formation and alcohol activation/elimination. This approach is also extended to terminal vinyl sulfonimidamides, about which very little is known. Both vinyl sulfoximines and sulfonimidamides can be readily $N$-functionalized with an alkyne tag. These products have the potential to be utilized as fully functionalized probes in a range of chemical biology applications.

Finally, we developed a computational workflow for assigning the configuration of vinyl sulfoximines and sulfonimidamides using ECD. After validating the approach against a vinyl sulfoxide with synthetically defined and known configuration, we apply the approach to the de novo assignment of enantiomeric and diastereoisomeric vinyl sulfoximines and vinyl sulfonimidamides. This methodology enables the implementation of chiral separation techniques to be applied to separate racemic vinyl sulfoximines and sulfonimidamides and assign their configuration, giving access to a range of new enantioenriched products. 
Taken together, the methods we have exemplified in this work provide the tools to access new areas of electrophilic chemical space. Our investigations into the application of vinyl sulfoximines and sulfonimidamides to chemical biology are currently underway.

\section{EXPERIMENTAL SECTION}

General experimental considerations. All non-aqueous reactions were carried out under an inert atmosphere (argon) with flame-dried glassware, using standard techniques. Anhydrous solvents were obtained by filtration through drying columns $\left(\mathrm{THF}, \mathrm{CH}_{2} \mathrm{Cl} 2\right.$, toluene). Forward phase flash column chromatography was performed on an Isolera ${ }^{\mathrm{TM}}$ Spektra flash purification system using Biotage ${ }^{\circledR}$ SNAP KP-Sil flash purification cartridges or SNAP Ultra flash purification cartridges, with the indicated solvent gradient. Reversed-phase flash column chromatography was performed using Biotage ${ }^{\circledR}$ SNAP Ultra C18 cartridges. Analytical thin-layer chromatography (TLC) was performed on precoated aluminium-backed silica gel plates. Visualisation of the developed chromatogram was performed by UV absorbance (254 nm) and/or stained with aqueous potassium permanganate solution, aqueous ceric ammonium molybdate, or a ninhydrin solution in ethanol. Nuclear magnetic resonance spectra were recorded on a $400 \mathrm{MHz}$ spectrometer. Chemical shifts for ${ }^{1} \mathrm{H}$ NMR spectra are recorded in parts per million from tetramethylsilane with the residual protic solvent resonance as the internal standard (chloroform: $\delta 7.27 \mathrm{ppm}, \mathrm{methanol}$ : $\delta 3.31 \mathrm{ppm}$ ). Data are reported as follows: chemical shift (multiplicity [s = singlet, $\mathrm{d}=\mathrm{doublet}, \mathrm{t}=$ triplet, $\mathrm{m}=$ multiplet and br $=\mathrm{broad}$ ], coupling constant (in Hz), integration). ${ }^{13} \mathrm{C}$ NMR spectra are recorded with complete proton decoupling. Chemical shifts are reported in parts per million from tetramethylsilane with the solvent resonance as the internal standard $\left.{ }^{13} \mathrm{CDCl}_{3}: \delta 77.0 \mathrm{ppm},{ }^{13} \mathrm{CD}_{3} \mathrm{OD}: \delta 49.0 \mathrm{ppm}\right) .{ }^{19} \mathrm{~F} \mathrm{NMR}$ spectra were recorded with complete proton decoupling. Chemical shifts are reported in parts per million, referenced to fluorobenzene as a standard at $\delta-113.5 \mathrm{ppm}$. Commercial reagents were used as supplied or purified by standard techniques where necessary. Optical rotations $\left(\alpha^{\prime}\right)$ were recorded at the indicated temperature $\left(\mathrm{T}^{\circ} \mathrm{C}\right)$ and were converted to the corresponding specific rotations $[\alpha]_{D}^{T}$.

General Procedure A: Preparation of NH Sulfoximines from sulfides by one-pot $N$ - and O-transfer. Procedure adapted from Tota et al. ${ }^{31 a}$ The sulfide (1.0 equiv), (diacetoxyiodo) benzene ( 2.5 equiv) and ammonium carbamate (2.0 equiv) were added to a flask containing a stirrer bar. $\mathrm{MeOH}(0.5 \mathrm{M})$ was added and the reaction was stirred at $25^{\circ} \mathrm{C}$ for $3 \mathrm{~h}$. The solvent was removed under reduced pressure. Purification by flash chromatography afforded the sulfoximine product.

General Procedure B: TBDPS protection of sulfoximine. A solution of imidazole (2.0 equiv) in anhydrous DMF was added to sulfoximine (1.0 equiv). The reaction mixture was cooled to $0{ }^{\circ} \mathrm{C}$ and tert-butyldiphenylsilyl chloride (1.25 equiv) was added drop-wise to the mixture. The solution was heated to $60^{\circ} \mathrm{C}$ and stirred for $64 \mathrm{~h}$ under nitrogen after which the reaction was quenched with water (10 mL). The aqueous layer was extracted with $\mathrm{CH}_{2} \mathrm{Cl}_{2}(3 \times 20 \mathrm{~mL})$ and the organic extracts were washed with water $(10 \mathrm{~mL})$ and brine $(10 \mathrm{~mL})$. The combined organic extracts were then dried $\left(\mathrm{MgSO}_{4}\right)$, filtered and concentrated in vacuo. Purification by flash chromatography afforded the TBDPS protected sulfoximine product.

General Procedure C: Preparation of vinyl sulfoximines by a Horner-Wadsworth-Emmons reaction. $n$ BuLi (1.3M in hexane, 2.7 equiv) was added to a solution of diisopropylamine (1.5 equiv) in anhydrous THF at $-78^{\circ} \mathrm{C}$ under nitrogen. The resulting solution was warmed to room temperature over $30 \mathrm{~min}$, before being cooled to $-78^{\circ} \mathrm{C}$ again. The TBDPS protected sulfoximine (1.0 equiv) in anhydrous THF was added dropwise to the reaction mixture and stirred at $-78^{\circ} \mathrm{C}$ for a further $1 \mathrm{~h}$. Diethylchlorophosphate (1.3 equiv) was added dropwise. After 5 min, ${ }^{t} \mathrm{BuOK}(1.0 \mathrm{M}$ in THF, 1.3 equiv) was added and the mixture was stirred for $10 \mathrm{~min}$ after which a solution of aldehyde (2.0 equiv) in anhydrous THF was added. The solution was then allowed to warm to $0{ }^{\circ} \mathrm{C}$ and stirred for $2 \mathrm{~h}$. The reaction was quenched with $5 \% \mathrm{w} / \mathrm{w}$ aq. $\mathrm{H}_{3} \mathrm{PO}_{4}(10 \mathrm{~mL})$. The aqueous layer was extracted with $\mathrm{Et}_{2} \mathrm{O}(3 \times 20 \mathrm{~mL})$ and the organic extracts were washed with $5 \% \mathrm{w} / \mathrm{w}$ aq. $\mathrm{H}_{3} \mathrm{PO}_{4}(2 \times$ $20 \mathrm{~mL})$. The combined organic extracts were then dried $\left(\mathrm{MgSO}_{4}\right)$, filtered and concentrated in vacuo. Purification by flash chromatography afforded the TBDPS protected vinyl sulfoximine product.

General Procedure D: TBDPS deprotection of vinyl sulfoximines. TBAF (1.0 M in THF, 1.1 equiv) was added dropwise to a solution of TBDPS protected vinyl sulfoximine (1.0 equiv) dissolved in anhydrous THF under nitrogen at room temperature. The reaction mixture was monitored by TLC every hour until the reaction was complete. The reaction mixture was flushed through a silica plug with $\mathrm{Et}_{2} \mathrm{O}$ and concentrated in vacuo. Purification by flash chromatography afforded the $\mathrm{NH}$ vinyl sulfoximine product.

General Procedure E: Preparation of NH Sulfoximines from sulfoxides by one-pot N-transfer. Procedure adapted from Zenzola et al. 30 The sulfide (1.0 equiv), (diacetoxyiodo)benzene (3.0 equiv) and ammonium carbamate (4.0 equiv) were added to a flask containing a stirrer bar. $\mathrm{MeOH}(0.5 \mathrm{M})$ was added and the reaction was stirred at $25^{\circ} \mathrm{C}$ for $3 \mathrm{~h}$. The solvent was removed under reduced pressure. Purification by flash chromatography afforded the sulfoximine product.

General Procedure F: Alkyne hydrothiolation. Procedure adapted from Shoai et al. ${ }^{40} \mathrm{RhCl}\left(\mathrm{PPh}_{3}\right)_{3}(5 \mathrm{~mol} \%)$ and any other solid reagents (thiol (1.1 equiv)/alkyne (1.0 equiv)) were added to a microwave vial and sealed. The flask was evacuated and filled with $\mathrm{N}_{2}$ three times before adding any liquid reagents (thiol (1.1 equiv)/alkyne (1.0 equiv)) and 1,2-dichloroethane ( $0.1 \mathrm{M})$ at $25^{\circ} \mathrm{C}$. The flask was left to stir for $18 \mathrm{~h}$ before the solvent was removed under reduced pressure. Purification by flash column chromatography afforded the corresponding vinyl sulfide product.

General Procedure G: Terminal vinyl sulfoximine synthesis by alcohol mesylation and in situ elimination. $\left.\mathrm{Mesyl}_{\mathrm{Chloride}}\left(1 \mathrm{M} \text { in } \mathrm{CH}_{2} \mathrm{Cl}\right)_{2}\right)(1.05$ equiv) was added dropwise to a stirred solution of triethylamine (4.0 equiv) and alcohol (1.0 equiv) in $\mathrm{CH}_{2} \mathrm{Cl}_{2}(0.1 \mathrm{M})$ at $0{ }^{\circ} \mathrm{C}$ and left to warm to room temperature. After $1 \mathrm{~h}$ the solvent was removed under reduced pressure. Purification by flash column chromatography afforded the corresponding vinyl $N \mathrm{H}$ sulfoximine and in some cases $N$-mesylated vinyl sulfoximine products.

General Procedure H: Synthesis of sulfonimidamides from amines via a sulfenamide intermediate.

Procedure adapted from Briggs et al. ${ }^{33}$ Amine (1.33 equiv), triethylamine (2.0-3.5 equiv) and silver nitrate (2.0 equiv) were added to a stirred solution of disulfide 16 (1.0 equiv) in $\mathrm{MeOH}(0.1 \mathrm{M})$ at $25^{\circ} \mathrm{C}$, under air, for $1 \mathrm{~h}$. The reaction mixture was filtered and washed with $\mathrm{MeOH}(2 \times 50 \mathrm{~mL})$. The filtrate solvent was removed under reduced pressure. $\mathrm{H}_{2} \mathrm{O}(75 \mathrm{~mL})$ was added to the crude mixture and the aqueous mixture extracted with $\mathrm{Et}_{2} \mathrm{O}(3 \times 75 \mathrm{~mL})$. The combined organic layers were dried $\left(\mathrm{Na}_{2} \mathrm{SO}_{4}\right)$, filtered and the solvent removed under reduced pressure. Purification by filtration through a basic alumina (activity IV) plug ( $2 \mathrm{~cm}$ thick) afforded the corresponding crude sulfenamide intermediate. Iodosylbenzene ( 2.5 equiv) and ammonium carbamate ( 2.0 equiv) were added to the crude sulfenamide (1.0 equiv) in a flask containing a stirrer bar under an argon atmosphere. A solution of acetic acid ( $0.2 \mathrm{M}$ solution, corresponding to $1.0 \mathrm{equiv}$ of $\mathrm{AcOH})$ in $i \mathrm{PrOH}$ $(0.2 \mathrm{M})$ was added and the reaction was stirred for $1 \mathrm{~h}$. The solvent was then removed under reduced pressure and to the crude, sat. aq. $\mathrm{NaHCO}_{3}$ was added $(20 \mathrm{~mL})$. The aqueous mixture was then extracted with $\mathrm{CH}_{2} \mathrm{Cl}_{2}(3 \times 20 \mathrm{~mL})$ and the combined organic layers were dried $\left(\mathrm{Na}_{2} \mathrm{SO}_{4}\right)$, filtered and the solvent removed under reduced pressure. Purification by flash chromatography afforded the corresponding sulfonimidamide products.

General Procedure I: Vinyl sulfonimidamides synthesis by THP deprotection and alcohol elimination. 
$\mathrm{HCl}\left(37 \%\right.$ in $\mathrm{H}_{2} \mathrm{O}, 1 \mathrm{~mL}$ ) was added to a stirred solution of sulfonimidamide (1 equiv) in MeOH:THF $(1: 1,12 \mathrm{~mL})$ at $25^{\circ} \mathrm{C}$. After $30 \mathrm{~min}$ sat. aq. $\mathrm{NaHCO}_{3}$ was added $(25 \mathrm{~mL})$. The aqueous mixture was then extracted with EtOAc $(3 \times 50 \mathrm{~mL})$ and the combined organic layers were dried $\left(\mathrm{Na}_{2} \mathrm{SO}_{4}\right)$, filtered and the solvent removed in vacuo to afford the crude alcohol intermediate. A solution of $\mathrm{MsCl}\left(1 \mathrm{M}\right.$ in $\mathrm{CH}_{2} \mathrm{Cl} 2,1.05$ equiv) was added dropwise to a solution of the crude alcohol (1.0 equiv) and triethylamine (4.0 equiv) in $\mathrm{CH}_{2} \mathrm{Cl}_{2}(0.1 \mathrm{M})$ at $0{ }^{\circ} \mathrm{C}$ and left to warm to room temperature for $16 \mathrm{~h}$. The solvent was removed in vacuo and purification by flash chromatography afforded the corresponding vinyl sulfonimidamide products.

General Procedure J: N-Propargylation of vinyl sulfoximines and sulfonimidamides. NaH (60\% in mineral oil, 1.2 equiv) was added to a solution of the sulfonimidamide $\left(1.0\right.$ equiv) in DMF $(0.1 \mathrm{M})$ at $0{ }^{\circ} \mathrm{C}$ and the reaction stirred for $30 \mathrm{~min}$. Propargyl bromide $(80 \%$ in toluene 1.5 equiv) was added and the reaction allowed to warm to room temperature for $3 \mathrm{~h} . \mathrm{H}_{2} \mathrm{O}(10 \mathrm{~mL})$ was added and the aqueous mixture extracted with EtOAc $(3 \times 20 \mathrm{~mL})$. The combined organic layers were dried $\left(\mathrm{Na}_{2} \mathrm{SO}_{4}\right)$, filtered and the solvent removed in vacuo. Purification flash chromatography afforded the corresponding $N$-propargyl sulfoximine or sulfonimidamide products.

\section{Substituted vinyl sulfoximines $\mathbf{5 a - 5 g}$}

Imino(methyl)(phenyl)- $\lambda 6$-sulfanone (2a). The title compound was prepared according to General Procedure A employing diacetoxyiodobenzene (3.90 g, $12.1 \mathrm{mmol})$, ammonium carbamate (753 mg, $9.66 \mathrm{mmol}$ ) and thioanisole $(600 \mathrm{mg}, 4.83 \mathrm{mmol}) \mathrm{in} \mathrm{MeOH} \mathrm{(9.6} \mathrm{mL).} \mathrm{Purifica-}$ tion via flash chromatography (KP-Sil, 0\% grading to 100\% EtOAc/Pentane, product eluted at 100\%) afforded 2a as a yellow oil (644 mg, 86\%); $\mathrm{R}_{\mathrm{f}}=0.42\left(100 \%\right.$ EtOAc); ${ }^{1} \mathrm{H}$ NMR $\left(400 \mathrm{MHz}, \mathrm{CDCl}_{3}\right) \delta 7.97-7.95(\mathrm{~m}, 2 \mathrm{H}), 7.58-7.55(\mathrm{~m}, 1 \mathrm{H}), 7.51-7.48(\mathrm{~m}, 1 \mathrm{H}), 3.06(\mathrm{~s}, 3 \mathrm{H}), 2.02(\mathrm{~s}$, $1 \mathrm{H}) ;{ }^{13} \mathrm{C}\left\{{ }^{1} \mathrm{H}\right\}$ NMR $\left(101 \mathrm{~Hz}, \mathrm{CDCl}_{3}\right) \delta 143.4,133.1,129.3(2 \times \mathrm{C}), 127.7(2 \times \mathrm{C}), 46.0 ;$ HRMS (ESI-TOF) m/z calcd C $7 \mathrm{H}_{11} \mathrm{NOS}^{+}\left[\mathrm{M}+\mathrm{H}^{+}, 156.0478\right.$; found, 156.0482. Analytical data in agreement with those reported in the literature. ${ }^{33}$

Imino(4-methoxyphenyl)(methyl)- $\lambda{ }^{6}$-sulfanone (2b). The title compound was prepared according to General Procedure A employing diacetoxyiodobenzene (10.4 g, $32.4 \mathrm{mmol})$, ammonium carbamate $(2.02 \mathrm{~g}, 25.9 \mathrm{mmol})$ and (4-methoxyphenyl)(methyl)sulfane (2.0 g, 13.0 $\mathrm{mmol}$ ) in $\mathrm{MeOH}(32 \mathrm{~mL}$ ). Purification via flash chromatography (KP-Sil, $0 \%$ grading to $100 \%$ EtOAc/Pentane, product eluted at $100 \%$ ) to afford $2 \mathbf{b}(1.70 \mathrm{~g}, 71 \%)$ as an orange oil; $\mathrm{R}_{\mathrm{f}}=0.21\left(100 \%\right.$ EtOAc); ${ }^{1} \mathrm{H}$ NMR $\left(400 \mathrm{MHz}, \mathrm{CDCl}_{3}\right) \delta 7.93-7.91(\mathrm{~d}, J=8.9 \mathrm{~Hz}, 2 \mathrm{H}), 7.00-6.98(\mathrm{~d}, J=$ $8.9 \mathrm{~Hz}, 2 \mathrm{H}), 3.87(\mathrm{~s}, 3 \mathrm{H}), 3.08(\mathrm{~s}, 3 \mathrm{H}) ;{ }^{13} \mathrm{C}\left\{{ }^{1} \mathrm{H}\right\} \mathrm{NMR}\left(101 \mathrm{~Hz}, \mathrm{CDCl}_{3}\right) \delta 163.3,134.9,129.88(2 \times \mathrm{C}), 114.4(2 \times \mathrm{C}), 55.7,46.5 ; \mathrm{HRMS}(\mathrm{ESI}-\mathrm{TOF})$ $m / z$ calcd for $\mathrm{C}_{8} \mathrm{H}_{12} \mathrm{NO}_{2} \mathrm{~S}^{+}[\mathrm{M}+\mathrm{H}]^{+}, 185.0510$; found, 185.0511. Analytical data in agreement with those reported in the literature. ${ }^{56}$

tert-Butyl (methyl(oxo)(phenyl)- $\lambda^{6}$-sulfaneylidene)carbamate (3a). The title compound was prepared according to General Procedure B employing sulfoximine $2 \mathrm{a}$ (400 mg, $2.58 \mathrm{mmol}$ ). Purification via flash chromatography (KP-Sil, $0 \%$ grading to $10 \%$ EtOAc/Pentane, product eluted at $8 \%)$ afforded sulfoximine $3 \mathbf{a}(578 \mathrm{mg}, 57 \%)$ as a clear oil; $\mathrm{R}_{\mathrm{f}}=0.20(15 \% \mathrm{EtOAc} /$ Pentane $) ;{ }^{1} \mathrm{H} \mathrm{NMR}(400 \mathrm{MHz}, \mathrm{CDCl}) \delta 7.87-7.85$ (m, 2H), 7.71-7.69 (m, 2H) 7.65-7.63 (m, 2H), 7.46-7.42 (m, 1H) 7.39-7.35 (m, 2H), 7.30-7.21 (m, 6H), $2.78(\mathrm{~s}, 3 \mathrm{H}) 1.02(\mathrm{~s}, 9 \mathrm{H}) ;{ }^{13} \mathrm{C}\left\{{ }^{1} \mathrm{H}\right\}$ $\operatorname{NMR}\left(101 \mathrm{~Hz}, \mathrm{CDCl}_{3}\right) \delta 144.5,136.23,136.19,135.64(2 \times \mathrm{C}), 135.60(2 \times \mathrm{C}), 132.2,129.1,128.9,128.9(2 \times \mathrm{C}), 127.44(2 \times \mathrm{C}), 127.41(2 \times$ C), 127.0, $(2 \times \mathrm{C}), 49.0,27.2,19.41(3 \times \mathrm{C})$. HRMS (ESI-TOF) $\mathrm{m} / \mathrm{z}$ calcd for $\mathrm{C}_{23} \mathrm{H}_{29} \mathrm{NOSiS}^{+}[\mathrm{M}+\mathrm{H}]^{+}, 394.1661$; found, 394.1657. Analytical data in agreement with those reported in the literature. 57

((tert-Butyldiphenylsilyl)imino)(4-methoxyphenyl)(methyl)- $\lambda$-sulfanone (3b). The title compound was prepared according to General Procedure B employing sulfoximine $\mathbf{2 b}(1.70 \mathrm{~g}, 2.58 \mathrm{mmol})$. Purification via flash chromatography (KP-Sil, $0 \%$ grading to $20 \%$ Et0Ac/Pentane, product eluted at $15 \%$ ) afforded sulfoximine $3 \mathbf{b}(447 \mathrm{mg}, 41 \%)$ as an off-white solid; $\mathrm{R}_{\mathrm{f}}=0.20$ (10\% EtOAc/Pentane); mp (chloroform) $=83-85^{\circ} \mathrm{C}$; IR (film) $/ \mathrm{cm}^{-1} 3071,2929,2855,1595,1498,1308,1259,1155,1107,1029,958,835,772,700,674 ;{ }^{1} \mathrm{H} \mathrm{NMR}(400 \mathrm{MHz}, \mathrm{CDCl} 3)$ $\delta 7.85(\mathrm{~d}, J=9.1 \mathrm{~Hz}, 2 \mathrm{H}), 7.78-7.76(\mathrm{~m}, 2 \mathrm{H}), 7.73-7.71(\mathrm{~m}, 2 \mathrm{H}), 7.37-7.28(\mathrm{~m}, 6 \mathrm{H}), 3.85(\mathrm{~s}, 3 \mathrm{H}), 2.83(\mathrm{~s}, 3 \mathrm{H}), 1.09(\mathrm{~s}, 9 \mathrm{H}) ; 13 \mathrm{C}\left\{{ }^{1} \mathrm{H}\right\} \mathrm{NMR}(101$ $\left.\mathrm{Hz}, \mathrm{CDCl}_{3}\right) \delta 162.6,136.6,136.5,136.4,135.7(2 \times \mathrm{C}), 135.6(2 \times \mathrm{C}) 129.1(1 \times \mathrm{C}), 129.0,129.1,127.42(3 \times \mathrm{C}), 127.38(3 \times \mathrm{C}), 113.9(2 \times \mathrm{C})$, 55.6, 49.4, 27.2, $19.4(3 \times \mathrm{C})$; HRMS (ESI-TOF) $\mathrm{m} / z$ Calcd for $\mathrm{C}_{24} \mathrm{H}_{30} \mathrm{NO} \mathrm{SSSi}^{+}[\mathrm{M}+\mathrm{H}]^{+}, 424.1767$; found, 424.1771 .

(E)-((tert-Butyldiphenylsilyl)imino)(phenyl)(styryl)- $\lambda 6$-sulfanone (4a). The title compound was prepared according to General Procedure C employing sulfoximine 3a (200 mg, $0.51 \mathrm{mmol}$ ) in anhydrous THF (3.2 mL), $n$ BuLi (1.3 M in hexane, $1.05 \mathrm{~mL}, 1.37 \mathrm{mmol}$ ), diisopropylamine $(0.11 \mathrm{~mL}, 0.72 \mathrm{mmol})$ in THF $(3.2 \mathrm{~mL})$, diethylchlorophosphate $(0.14 \mathrm{~mL}, 0.66 \mathrm{mmol}), t \mathrm{BuOK}(1.0 \mathrm{M} \mathrm{in} \mathrm{THF,} 0.66 \mathrm{~mL}, 0.66 \mathrm{mmol})$, benzaldehyde $(0.10 \mathrm{~mL}, 1.02 \mathrm{mmol})$ in THF $(8 \mathrm{~mL})$. Purification via flash chromatography (KP-Sil, $0 \%$ grading to $100 \%$ EtOAc/Pentane, eluted at $9 \%$ EtOAc) afforded vinyl sulfoximine $4 \mathrm{a}(147 \mathrm{mg}, 60 \%)$ as a clear oil; $\mathrm{Rf}_{\mathrm{f}}=0.49$ (10\% EtOAc/Pentane); IR (film)/ $\mathrm{cm}^{-1} 3064,2930$, $2855,1677,1614,1293,1148,1107,969,816,745,700 ;{ }^{1} \mathrm{H}^{\mathrm{NMR}}\left(400 \mathrm{MHz}, \mathrm{CDCl}_{3}\right) \delta$ 7.87-7.85 (m, 2H), 7.71-7.65 (m, 4H), 7.44-7.40 (m, $1 \mathrm{H}), 7.37-7.33(\mathrm{~m}, 3 \mathrm{H}), 7.31(\mathrm{~d}, J=15.2 \mathrm{~Hz}, 1 \mathrm{H}), 7.26-7.20(\mathrm{~m}, 8 \mathrm{H}), 7.14-7.12(\mathrm{~m}, 2 \mathrm{H}), 6.53(\mathrm{~d}, J=15.2 \mathrm{~Hz}), 1.05(\mathrm{~s}, 9 \mathrm{H}) ;{ }^{13} \mathrm{C}\left\{{ }^{1} \mathrm{H}\right\} \mathrm{NMR}(101$ $\left.\mathrm{Hz}, \mathrm{CDCl}_{3}\right) \delta 144.3,138.9,136.4,136.3,135.69(2 \times \mathrm{C}), 135.66(2 \times \mathrm{C}), 134.8,133.0,132.0,131.9,130.3(2 \times \mathrm{C}), 128.9(2 \times \mathrm{C}), 128.8(3 \times \mathrm{C})$, $128.3(2 \times \mathrm{C}), 27.2,19.5$; HRMS (ESI-TOF) $\mathrm{m} / z$ Calcd for $\mathrm{C}_{30} \mathrm{H}_{32} \mathrm{NOSiS}^{+}[\mathrm{M}+\mathrm{H}]^{+}$, 482.1974; found, 482.1982 .

(E)-((tert-Butyldiphenylsilyl)imino)(2-cyclohexylvinyl)(phenyl)- $\lambda^{6}$-sulfanone (4b). The title compound was prepared according to General Procedure C employing sulfoximine $3 a(200 \mathrm{mg}, 0.51 \mathrm{mmol})$ in anhydrous THF ( $3.2 \mathrm{~mL}), n$ BuLi $(1.3 \mathrm{M}$ in hexane, $1.05 \mathrm{~mL}, 1.37 \mathrm{mmol})$, diisopropylamine $(0.1 \mathrm{~mL}, 0.77 \mathrm{mmol})$ in anhydrous THF $(3.2 \mathrm{~mL})$, diethylchlorophosphate $(0.09 \mathrm{~mL}, 0.66 \mathrm{mmol}), t B u$ OK $(1.0 \mathrm{M}$ in THF, 0.66 $\mathrm{mL}, 0.66 \mathrm{mmol})$, cyclohexanecarboxaldehyde $(0.12 \mathrm{~mL}, 1.02 \mathrm{mmol})$ in anhydrous THF $(8.0 \mathrm{~mL})$. Purification via flash chromatography (KPSil, $0 \%$ grading to $10 \%$ EtOAc/Pentane, eluted at $7 \%$ EtOAc), afforded $4 \mathbf{b}(142 \mathrm{mg}, 57 \%)$ as a clear yellow oil; $\mathrm{R}_{\mathrm{f}}=0.66$ (10\% EtOAc/Pentane); IR (film)/ $\mathrm{cm}^{-1} 3067,2929,2855,1319,1159,1107,820,745,700 ;{ }^{1} \mathrm{H}$ NMR $\left(400 \mathrm{MHz}, \mathrm{CDCl}_{3}\right) \delta 7.80-7.78(\mathrm{~m}, 2 \mathrm{H}), 7.68-7.63(\mathrm{~m}, 4 \mathrm{H}), 7.42-$ $7.38(\mathrm{~m}, 1 \mathrm{H}), 7.35-7.32(\mathrm{~m}, 2 \mathrm{H}), 7.29-7.21(\mathrm{~m}, 6 \mathrm{H}), 6.62-6.57(\mathrm{dd}, J=6.2,8.6 \mathrm{~Hz}), 5.98-5.94(\mathrm{dd}, J=1.5,13.4 \mathrm{~Hz}), 1.89-1.82(\mathrm{~m}, 2 \mathrm{H}), 1.60-$ $1.45(\mathrm{~m}, 6 \mathrm{H}), 1.18-1.05(\mathrm{~m}, 2 \mathrm{H}), 1.02(\mathrm{~s}, 9 \mathrm{H}), 0.89-0.76(\mathrm{~m}, 2 \mathrm{H}) ;{ }^{13} \mathrm{C}\left\{{ }^{1} \mathrm{H}\right\} \mathrm{NMR}\left(101 \mathrm{~Hz}, \mathrm{CDCl}_{3}\right) \delta 147.9,144.6,136.6,136.5,135.7(2 \times \mathrm{C})$, $135.6(2 \times \mathrm{C}), 132.3(2 \times \mathrm{C}), 131.7,128.9(2 \times \mathrm{C}), 128.7(3 \times \mathrm{C}), 127.3(2 \times \mathrm{C}), 127.2(2 \times \mathrm{C}), 39.3,31.3(2 \times \mathrm{C}), 27.2(3 \times \mathrm{C}), 25.8(2 \times \mathrm{C}), 25.5$, 19.5; HRMS (ESI-TOF) $\mathrm{m} / z$ Calcd for $\mathrm{C}_{30} \mathrm{H}_{38} \mathrm{NOSSi}^{+}[\mathrm{M}+\mathrm{H}]^{+}, 488.2443$; found, 488.2447 .

(E)-((tert-Butyldiphenylsilyl)imino)(4-ethynylstyryl)(phenyl)- $\lambda 6$-sulfanone (4c). The title compound was prepared according to General Procedure C employing sulfoximine 3a (600 mg, $0.51 \mathrm{mmol})$ in THF ( $9.6 \mathrm{~mL}), n B u L i ~(1.3 \mathrm{M}$ in hexane, $3.10 \mathrm{~mL}, 4.14 \mathrm{mmol})$, diisopropylamine $(0.30 \mathrm{~mL}, 2.25 \mathrm{mmol})$ in THF $(9.6 \mathrm{~mL})$, diethylchlorophosphate $(0.30 \mathrm{~mL}, 1.98 \mathrm{mmol}), t \mathrm{BuOK}(1.0 \mathrm{M}$ in THF, $1.98 \mathrm{~mL}, 1.98 \mathrm{mmol})$ and 4 ethynylbenzaldehyde (392 mg, $3.04 \mathrm{mmol})$ in THF $(20 \mathrm{~mL})$. Purification via flash chromatography (KP-Sil, $0 \%$ grading to $10 \%$ EtOAc/Pentane, eluted at $8 \%$ EtOAc), afforded $4 \mathbf{c}(175 \mathrm{mg}, 68 \%)$ as an orange oil; IR (film)/cm-13067, 2925, 2851, 1325, 1160, 1107, 821, 745; ${ }^{1} \mathrm{H}$ NMR $\left(400 \mathrm{MHz}, \mathrm{CDCl}_{3}\right) \delta$ 7.95-7.93 (m, 2H), 7.77-7.72 (m, 4H), 7.54-7.48 (m, 1H), 7.46-7.44 (m, 2H), 7.41-7.38 (m, 2H), 7.34-7.27 (m, 6H), 7.13$7.11(\mathrm{~m}, 2 \mathrm{H}), 6.57(\mathrm{~d}, J=15.1 \mathrm{~Hz}, 1 \mathrm{H}), 3.16(\mathrm{~s}, 1 \mathrm{H}), 1.13(\mathrm{~s}, 9 \mathrm{H}) ;{ }^{13} \mathrm{C}\left\{{ }^{1} \mathrm{H}\right\} \mathrm{NMR}\left(101 \mathrm{~Hz}, \mathrm{CDCl}_{3}\right) \delta 144.1,137.6,136.3,136.2,135.69(2 \times \mathrm{C})$, $135.66(2 \times \mathrm{C}), 133.4,132.8,132.4(2 \times \mathrm{C}), 132.2,129.0(2 \times \mathrm{C}), 128.9(2 \times \mathrm{C}), 128.1(2 \times \mathrm{C}), 127.5(2 \times \mathrm{C}), 127.43(2 \times \mathrm{C}), 127.39(2 \times \mathrm{C})$, 123.9, 83.0, 79.2, 27.2, 19.5; HRMS (ESI-TOF) $m / z$ Calcd for $\mathrm{C}_{32} \mathrm{H}_{32} \mathrm{NOSSi}^{+}[\mathrm{M}+\mathrm{H}]^{+}, 506.1968$; found, 506.1970. 
(E)-((tert-Butyldiphenylsilyl)imino)(phenyl)(2-(pyridin-2-yl)vinyl)- $\lambda$ 6-sulfanone (4d). The title compound was prepared according to General Procedure C employing sulfoximine 3a (200 mg, $0.51 \mathrm{mmol})$ in anhydrous THF (3.2 mL), $n$ BuLi (1.3 M in hexane, $1.05 \mathrm{~mL}, 1.37$ $\mathrm{mmol})$, diisopropylamine $(0.10 \mathrm{~mL}, 0.77 \mathrm{mmol})$ in THF $(3.2 \mathrm{~mL})$, diethylchlorophosphate $(0.09 \mathrm{~mL}, 0.66 \mathrm{mmol}), t \mathrm{BuOK}(1.0 \mathrm{M}$ in THF, 0.66 $\mathrm{mL}, 0.66 \mathrm{mmol})$, picolinaldehyde $(0.12 \mathrm{~mL}, 1.02 \mathrm{mmol})$ in THF ( $8 \mathrm{~mL})$. Purification via flash chromatography (KP-Sil, $0 \%$ grading to $20 \%$ EtOAc/Petroleum ether, eluted at 20\% EtOAc), afforded 4d (148 mg, 60\%) as a yellow oil; $\mathrm{R}_{\mathrm{f}}=0.19(10 \%$ EtOAc/Petroleum ether); IR (film) $/ \mathrm{cm}^{-1} 3067,2929,2855,1580,1468,1427,1327,1170,1107,820,704 ;{ }^{1} \mathrm{H} \mathrm{NMR}\left(400 \mathrm{MHz}, \mathrm{CDCl}_{3}\right) \delta 8.54-8.53(\mathrm{~d}, J=4.6 \mathrm{~Hz}, 1 \mathrm{H}), 7.97-$ $7.94(\mathrm{~m}, \mathrm{H}), 7.80-7.74(\mathrm{~m}, 4 \mathrm{H}), 7.64-7.60(\mathrm{~m}, 1 \mathrm{H}), 7.50-7.44(\mathrm{~m}, 1 \mathrm{H}), 7.40-7.36(\mathrm{~m}, 2 \mathrm{H}), 7.32(\mathrm{~d}, J=15.2 \mathrm{~Hz}), 7.32-7.27(\mathrm{~m}, 5 \mathrm{H}), 7.25-7.18$ $(\mathrm{m}, 2 \mathrm{H}), 7.13-7.11(\mathrm{~d}, J=7.8 \mathrm{~Hz}, 1 \mathrm{H}), 1.14(\mathrm{~s}, 9 \mathrm{H}) .{ }^{13} \mathrm{C}\left\{{ }^{1} \mathrm{H}\right\} \mathrm{NMR}\left(101 \mathrm{~Hz}, \mathrm{CDCl}_{3}\right) \delta 151.9,150.0,143.8,137.1,136.7,136.3,135.7(2 \times \mathrm{C}), 135.7$ $(2 \times \mathrm{C}), 132.2,128.9(3 \times \mathrm{C}), 127.6(2 \times \mathrm{C}), 127.4(2 \times \mathrm{C}), 127.3(2 \times \mathrm{C}), 124.8,124.2,27.3(3 \times \mathrm{C}), 19.5$. HRMS (ESI-TOF) $m / z$ Calcd for $\mathrm{C}_{29} \mathrm{H}_{31} \mathrm{~N}_{2} \mathrm{OSSi}^{+}[\mathrm{M}+\mathrm{H}]^{+}, 483.1926$; found: 483.1931 .

(E)-((tert-Butyldiphenylsilyl)imino)(4-ethynylstyryl)(4-methoxyphenyl)- $\lambda^{6}$-sulfanone (4e). The title compound was prepared according to General Procedure C employing sulfoximine $\mathbf{3 b}(200 \mathrm{mg}, 0.47 \mathrm{mmol})$ in THF (3.2 mL), $n$ BuLi $(1.3 \mathrm{M}$ in hexane, $1.05 \mathrm{~mL}, 1.37 \mathrm{mmol})$, diisopropylamine $(0.10 \mathrm{~mL}, 0.77 \mathrm{mmol})$ in THF $(3.2 \mathrm{~mL})$, diethylchlorophosphate $(0.09 \mathrm{~mL}, 0.66 \mathrm{mmol}), t \mathrm{BuOK}(1.0 \mathrm{M} \mathrm{in} \mathrm{THF,} 0.66 \mathrm{~mL}, 0.66$ $\mathrm{mmol}$ ), ethynylbenzaldehyde $(123 \mathrm{mg}, 0.94 \mathrm{mmol}$ ) in THF ( $8 \mathrm{~mL}$ ). Purification via flash chromatography (KP-Sil, $0 \%$ grading to $20 \%$ EtOAc/Petroleum ether, eluted at $12 \%$ EtOAc) afforded $4 \mathbf{e}(128 \mathrm{mg}, 51 \%)$ as a clear oil; $\mathrm{R}_{\mathrm{f}}=0.35(20 \%$ EtOAc$/ \mathrm{Pentane}) ; \mathrm{IR}$ (film) $/ \mathrm{cm}^{-1} 3291$, 2929, 2855, 1595, 1495, 1312, 1259, 1148, 1107, 1028, 805, 704; ${ }^{1} \mathrm{H}$ NMR $\left(400 \mathrm{MHz}, \mathrm{CDCl}_{3}\right) \delta 7.85(\mathrm{~d}, J=8.9 \mathrm{~Hz}, 2 \mathrm{H}), 7.77-7.71(\mathrm{~m}, 4 \mathrm{H})$, $7.37(\mathrm{~d}, J=8.4 \mathrm{~Hz}, 2 \mathrm{H}), 7.35-7.24(\mathrm{~m}, 7 \mathrm{H}), 7.09(\mathrm{~d}, J=8.4 \mathrm{~Hz}, 2 \mathrm{H}), 6.90(\mathrm{~d}, J=8.9 \mathrm{~Hz}, 2 \mathrm{H}), 6.56(\mathrm{~d}, J=15.2 \mathrm{~Hz}, 1 \mathrm{H}), 3.84(\mathrm{~s}, 3 \mathrm{H}), 3.16(\mathrm{~s}, 3 \mathrm{H})$, $1.12(\mathrm{~s}, 9 \mathrm{H}) ;{ }^{13} \mathrm{C}\left\{{ }^{1} \mathrm{H}\right\} \mathrm{NMR}\left(101 \mathrm{~Hz}, \mathrm{CDCl}_{3}\right) \delta 162.6(2 \times \mathrm{C}), 136.7,136.5,136.3,135.8,135.7(3 \times \mathrm{C}), 133.5,133.4,132.4(2 \times \mathrm{C}), 129.6(2 \times \mathrm{C})$,

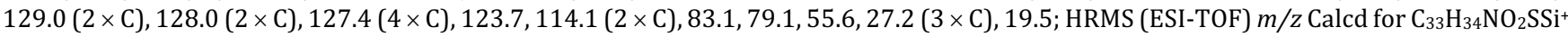
$[\mathrm{M}+\mathrm{H}]^{+}$, 536.2080; found: 536.2074 .

(E)-((tert-Butyldiphenylsilyl)imino)(4-methoxyphenyl)(2-(thiazol-2-yl)vinyl)- $\lambda 6$-sulfanone (4f). The title compound was prepared according to General Procedure C employing sulfoximine $\mathbf{3 b}(300 \mathrm{mg}, 0.71 \mathrm{mmol})$ in THF (4.8 mL), $n$ BuLi $(1.3 \mathrm{M}$ in hexane, $1.50 \mathrm{~mL}, 1.91$ $\mathrm{mmol})$, diisopropylamine $(0.15 \mathrm{~mL}, 1.07 \mathrm{mmol})$ in THF $(4.8 \mathrm{~mL})$, diethylchlorophosphate $(0.13 \mathrm{~mL}, 0.92 \mathrm{mmol}), t \mathrm{BuOK}(1.0 \mathrm{M} \mathrm{in} \mathrm{THF}, 0.92$ $\mathrm{mL}, 0.92 \mathrm{mmol}), 1,3$ thiazole -2 -carbaldehyde $(0.13 \mathrm{~mL}, 1.42 \mathrm{mmol})$ in THF $(10 \mathrm{~mL})$. Purification via flash chromatography (KP-Sil, $0 \%$ grading to $50 \%$ EtOAc/Hexane, eluted at $30 \%$ EtOAc) afforded $\mathbf{4 f}(311 \mathrm{mg}, 84 \%)$ as a yellow oil; Rf $=0.38(\mathrm{EtOAc} / \mathrm{Hexane}) ; \mathrm{IR}(\mathrm{film}) / \mathrm{cm}^{-1}$ $3071,2959,2855,1595,1494,1312,1259,1151,1107,1028,820,704 ;{ }^{1} \mathrm{H} \mathrm{NMR}\left(400 \mathrm{MHz}, \mathrm{CDCl}_{3}\right) \delta 7.85(\mathrm{~d}, J=8.7 \mathrm{~Hz}, 2 \mathrm{H}), 7.81(\mathrm{~d}, J=3.2$ $\mathrm{Hz}, 1 \mathrm{H}), 7.78-7.72(\mathrm{~m}, 4 \mathrm{H}), 7.37(\mathrm{~d}, J=15.3 \mathrm{~Hz}), 7.35(\mathrm{~d}, J=3.32 \mathrm{~Hz}, 1 \mathrm{H}), 7.32-7.28(\mathrm{~m}, 6 \mathrm{H}), 6.96(\mathrm{~d}, J=15.3 \mathrm{~Hz}, 1 \mathrm{H}), 6.90(\mathrm{~d}, J=8.8 \mathrm{~Hz}, 2 \mathrm{H})$; ${ }^{13} \mathrm{C}\left\{{ }^{1} \mathrm{H}\right\}$ NMR $\left(101 \mathrm{~Hz}, \mathrm{CDCl}_{3}\right) \delta 162.9,162.0,144.5,137.1,136.1(2 \times \mathrm{C}), 135.7(4 \times \mathrm{C}), 135.0,129.9(2 \times \mathrm{C}), 129.0,128.7,127.4(6 \times \mathrm{C}), 121.4$, $114.2(2 \times \mathrm{C}), 55.6,27.3(3 \times \mathrm{C}), 19.5$; HRMS (ESI-TOF) $\mathrm{m} / z$ Calcd for $\mathrm{C}_{27} \mathrm{H}_{31} \mathrm{~N}_{2} \mathrm{OS}_{2} \mathrm{Si}^{+}[\mathrm{M}+\mathrm{H}]^{+}, 519.1596$; found, 519.1599 .

(E)-((tert-Butyldiphenylsilyl)imino)(4-methoxyphenyl)(2-(quinoxalin-2-yl)vinyl)- $\lambda^{6}$-sulfanone (4g). The title compound was prepared according to General Procedure C employing sulfoximine $3 \mathbf{b}(300 \mathrm{mg}, 0.71 \mathrm{mmol})$ in anhydrous THF (4.8 mL), $n \mathrm{BuLi}(1.3 \mathrm{M}$ in hexane, 1.50 $\mathrm{mL}, 1.91 \mathrm{mmol})$, diisopropylamine $(0.15 \mathrm{~mL}, 1.07 \mathrm{mmol})$ in THF $(4.8 \mathrm{~mL})$, diethylchlorophosphate $(0.13 \mathrm{~mL}, 0.92 \mathrm{mmol}), t \mathrm{BuOK}(1.0 \mathrm{M} \mathrm{in}$ THF, $0.92 \mathrm{~mL}, 0.92 \mathrm{mmol}$ ), quinoxaline-2-carbaldehyde (200 mg, $1.42 \mathrm{mmol})$ in THF (10 mL). Purification by flash chromatography (KP-Sil, $0 \%$ grading to $50 \%$ EtOAc/Hexane, eluted at $26 \%$ EtOAc) afforded $\mathbf{4 g}(271 \mathrm{mg}, 68 \%)$ as an orange oil; $\mathrm{R}_{\mathrm{f}}=0.33(40 \% \mathrm{EtOAc} / \mathrm{Hexane}) ; \mathrm{IR}$ (film)/cm ${ }^{-1} 3071,2963,2855,1595,1494,1312,1259,1148,1107,1058,820,764,704 ;{ }^{1} \mathrm{H} \mathrm{NMR}\left(400 \mathrm{MHz}, \mathrm{CDCl}_{3}\right) \delta 8.55(\mathrm{~s}, 1 \mathrm{H}), 8.07-8.05$ $(\mathrm{m}, 1 \mathrm{H}), 8.00-7.97(\mathrm{~m}, 1 \mathrm{H}), 7.93(\mathrm{~d}, J=8.9 \mathrm{~Hz}, 2 \mathrm{H}), 7.80-7.74(\mathrm{~m}, 6 \mathrm{H}), 7.38(\mathrm{~d}, J=15.3 \mathrm{~Hz}, 1 \mathrm{H}), 7.28(\mathrm{~d}, J=15.3 \mathrm{~Hz}, 1 \mathrm{H}), 7.28-7.26(\mathrm{~m}, 6 \mathrm{H})$, $6.94(\mathrm{~d}, J=8.81 \mathrm{~Hz}, 2 \mathrm{H}), 3.85(\mathrm{~s}, 3 \mathrm{H}), 1.15(\mathrm{~s}, 9 \mathrm{H}) ;{ }^{13} \mathrm{C}\left\{{ }^{1} \mathrm{H}\right\}$ NMR $\left(101 \mathrm{~Hz}, \mathrm{CDCl}_{3}\right) \delta 163.0,147.2,144.9,142.3(2 \times \mathrm{C}), 139.7,136.1,135.7(4 \times$ C), 134.9, 132.9, $130.7(2 \times \mathrm{C}), 130.1(2 \times \mathrm{C}), 129.6,129.2,129.1(2 \times \mathrm{C}), 127.4(6 \times \mathrm{C}), 114.3(2 \times \mathrm{C}), 55.7,27.3(3 \times \mathrm{C}), 19.5$; HRMS (ESI-TOF) $\mathrm{m} / z$ Calcd for $\mathrm{C}_{33} \mathrm{H}_{34} \mathrm{~N}_{3} \mathrm{O}_{2} \mathrm{SSi}^{+}[\mathrm{M}+\mathrm{H}]^{+}, 564.2141$; found, 564.2154 .

$(E)$-Imino(phenyl)(styryl)- $\lambda 6$-sulfanone (5a). The title compound was prepared according to General Procedure D employing vinyl sulfoximine $4 \mathrm{a}(125 \mathrm{mg}, 0.26 \mathrm{mmol})$ and TBAF (1.0 M in THF, $0.29 \mathrm{~mL}, 0.29 \mathrm{mmol}$ ) in anhydrous THF (4.7 mL). Purification via flash chromatography (KP-Sil, $0 \%$ grading to $100 \%$ EtOAc/Pentane, eluted at 58\% EtOAc) vinyl sulfoximine $\mathbf{5 a}$ as a yellow oil $(47 \mathrm{mg}, 74 \%) ; \mathrm{R}_{\mathrm{f}}=0.58$ $\left(100 \%\right.$ EtOAc); ${ }^{1} \mathrm{H}$ NMR $\left(400 \mathrm{MHz}, \mathrm{CDCl}_{3}\right) \delta 8.05-8.02(\mathrm{~m}, 2 \mathrm{H}), 7.62(\mathrm{~d}, J=15.2 \mathrm{~Hz}, 1 \mathrm{H}), 7.60-7.56(\mathrm{~m}, 1 \mathrm{H}), 7.55-7.51(\mathrm{~m}, 2 \mathrm{H}), 7.49-7.46(\mathrm{~m}$, 2H), 7.40-7.37 (m, 3H), $6.93(\mathrm{~d}, J=15.3 \mathrm{~Hz}, 1 \mathrm{H}), 2.68(\mathrm{bs}, 1 \mathrm{H}) ;{ }^{13} \mathrm{C}\left\{{ }^{1} \mathrm{H}\right\} \mathrm{NMR}\left(101 \mathrm{~Hz}, \mathrm{CDCl}_{3}\right) \delta 142.8,141.6,132.8,132.7,130.9,129.5(2 \times$ C), $129.2(2 \times \mathrm{C}), 129.0,128.5(2 \times \mathrm{C}), 127.9(2 \times \mathrm{C})$; HRMS (ESI-TOF) $\mathrm{m} / z$ Calcd for $\mathrm{C}_{14} \mathrm{H}_{14} \mathrm{NOS}^{+}\left[\mathrm{M}+\mathrm{H}^{+}, 244.0807\right.$; found, 244.0796 . Analytical data in agreement with those reported in the literature.22

(E)-(2-Cyclohexylvinyl)(imino)(phenyl)- $\lambda^{6}$-sulfanone (5b). The title compound was prepared according to General Procedure D employing vinyl sulfoximine $4 \mathbf{b}(115 \mathrm{mg}, 0.24 \mathrm{mmol})$ and TBAF (1.0 M in THF, $0.26 \mathrm{~mL}, 0.26 \mathrm{mmol})$ in THF (4.5 mL). Purification via flash chromatography (KP-Sil, $0 \%$ grading to $100 \%$ EtOAc/Pentane, eluted at $50 \%$ EtOAc) afforded $\mathbf{5 b}(44 \mathrm{mg}, 74 \%)$ as a yellow oil; $\mathrm{R}_{\mathrm{f}}=0.42(50 \%$ EtOAc/Pentane); IR (film)/ $\mathrm{cm}^{-1} 3270,2924,2851,1625,1446,1226,1125,969,7511,688 ;{ }^{1} \mathrm{H} \mathrm{NMR}\left(400 \mathrm{MHz}, \mathrm{CDCl}_{3}\right) \delta 7.96-7.93(\mathrm{~m}, 2 \mathrm{H})$, 7.59-7.55 (m, 1H), 7.52-7.48 (m, 2H), $6.86(\mathrm{dd}, J=8.7 \mathrm{~Hz}, 6.5 \mathrm{~Hz}), 6.30(\mathrm{dd}, J=14.1 \mathrm{~Hz}, 1.4 \mathrm{~Hz}, 1 \mathrm{H}), 2.44(\mathrm{bs}, 1 \mathrm{H}), 2.20-2.13(\mathrm{~m}, 1 \mathrm{H}), 1.75-$ $1.72(\mathrm{~m}, 4 \mathrm{H}), 1.67-1.64(\mathrm{~m}, 1 \mathrm{H}), 1.31-1.06(\mathrm{~m}, 5 \mathrm{H}) ;{ }^{13} \mathrm{C}\left\{{ }^{1} \mathrm{H}\right\} \mathrm{NMR}\left(101 \mathrm{~Hz}, \mathrm{CDCl}_{3}\right) \delta 150.7,143.1,132.6,130.0,129.10(2 \times \mathrm{C}), 127.7(2 \times \mathrm{C})$, $39.8(1 \times \mathrm{C}), 31.4,31.3,25.8(2 \times \mathrm{C}), 25.6(1 \times \mathrm{C})$; HRMS (ESI) $\mathrm{m} / z$ Calcd for $\mathrm{C}_{14} \mathrm{H}_{20} \mathrm{NOS}^{+}[\mathrm{M}+\mathrm{H}]^{+}, 250.1266$; found, 250.1263 .

$(E)$-(4-Ethynylstyryl)(imino)(phenyl)- $\lambda 6$-sulfanone

$(5 c)$.

The title compound was prepared according to General Procedure D employing vinyl sulfoximine 4c (690 mg, $1.37 \mathrm{mmol}$ ) and TBAF (1.0 M in THF, $50 \mathrm{~mL}, 1.51 \mathrm{mmol}$ ) in THF (25 mL). Purification via flash chromatography (KP-Sil, $0 \%$ grading to $100 \%$ EtOAc/Pentane, eluted at $50 \%$ EtOAc) afforded $\mathbf{5 c}(300 \mathrm{mg}, 82 \%)$ as a white solid; $\mathrm{R}_{\mathrm{f}}=0.43(50 \% \mathrm{EtOAc} /$ Pentane $) ; \mathrm{mp}\left(\mathrm{CHCl}_{3}\right) 133-135^{\circ} \mathrm{C} ; \mathrm{IR}(\mathrm{film}) / \mathrm{cm}^{-1} 3279,3061$, 2579, 2361, 1614, 1445, 1217, 1125, 974, 799, 753, 687; ${ }^{1 \mathrm{H}} \mathrm{NMR}\left(400 \mathrm{MHz}, \mathrm{CDCl}_{3}\right) \delta 8.04-8.02(\mathrm{~m}, 2 \mathrm{H}), 7.62-7.57(\mathrm{~m}, 2 \mathrm{H}), 7.56-7.52(\mathrm{~m}$, $2 \mathrm{H}), 7.47(\mathrm{~d}, J=8.5 \mathrm{~Hz}, 2 \mathrm{H}), 7.41(\mathrm{~d}, J=8.4 \mathrm{~Hz}, 2 \mathrm{H}), 6.92(\mathrm{~d}, J=15.3 \mathrm{~Hz}, 1 \mathrm{H}), 3.19(\mathrm{~s}, 1 \mathrm{H}), 2.95(\mathrm{bs}, 1 \mathrm{H}) ;{ }^{13} \mathrm{C}\left\{{ }^{1} \mathrm{H}\right\} \mathrm{NMR}(101 \mathrm{~Hz}, \mathrm{CDCl}) \delta 142.5$, 140.4, 133.0, $132.7(2 \times \mathrm{C}), 130.5,129.3(2 \times \mathrm{C}), 128.3(2 \times \mathrm{C}), 128.0(2 \times \mathrm{C}), 124.6,82.9,79.7$; HRMS (ESI-TOF) $m / z$ Calcd for C $16 \mathrm{H}_{14} \mathrm{NOS}^{+}[\mathrm{M}$ $+\mathrm{H}]^{+}, 268.0796$; found, 268.0804 .

(E)-Imino(phenyl)(2-(pyridin-2-yl)vinyl)- $\lambda^{6}$-sulfanone (5d). The title compound was prepared according to General Procedure D employing vinyl sulfoximine $4 \mathbf{d}(70 \mathrm{mg}, 0.15 \mathrm{mmol})$ and TBAF $(1.0 \mathrm{M}$ in THF, $0.16 \mathrm{~mL}, 0.16 \mathrm{mmol})$ in THF (2.8 mL). Purification via flash chromatography (KP-Sil, $0 \%$ grading to $100 \%$ EtOAc/Petroleum ether, eluted at $100 \%$ EtOAc) afforded $\mathbf{5 d}\left(25 \mathrm{mg}\right.$, $69 \%$ ) as a white solid; $\mathrm{R}_{\mathrm{f}}$ $=0.39\left(100 \%\right.$ EtOAc); $\mathrm{mp}\left(\mathrm{CHCl}_{3}\right)=94-96^{\circ} \mathrm{C} ; \mathrm{IR}(\mathrm{film}) / \mathrm{cm}^{-1} 3258,3056,1580,1469,1431,1215,1111,969,797,753,708 ;{ }^{1} \mathrm{H} \mathrm{NMR}(400$ $\left.\mathrm{MHz}, \mathrm{CDCl}_{3}\right) \delta 8.60-8.59(\mathrm{~d}, J=4.5 \mathrm{~Hz}, 1 \mathrm{H}), 8.06-8.04(\mathrm{~m}, 2 \mathrm{H}), 7.69(\mathrm{~m}, 1 \mathrm{H}), 7.60(\mathrm{~d}, J=15.2 \mathrm{~Hz}, 1 \mathrm{H}), 7.59-7.54(\mathrm{~m}, 1 \mathrm{H}), 7.54-7.51(\mathrm{~m}, 2 \mathrm{H})$, $7.46(\mathrm{~d}, J=15.3 \mathrm{~Hz}, 1 \mathrm{H}), 7.40-7.38(\mathrm{~d}, J=7.8 \mathrm{~Hz}, 1 \mathrm{H}) 2.67(\mathrm{bs}, 1 \mathrm{H}) ;{ }^{13} \mathrm{C}\left\{{ }^{1} \mathrm{H}\right\} \mathrm{NMR}(101 \mathrm{~Hz}, \mathrm{CDCl} 3) \delta 151.4,150.2,142.0,139.6,137.0,134.2$, $133.0(2 \times \mathrm{C}), 129.2(2 \times \mathrm{C}), 128.2,125.2,124.7$; HRMS (ESI-TOF) $\mathrm{m} / z$ Calcd for $\mathrm{C}_{13} \mathrm{H}_{13} \mathrm{~N}_{2} \mathrm{OS}+[\mathrm{M}+\mathrm{H}]^{+}, 245.0749$; found, 245.0755. Analytical data in agreement with those reported in the literature. ${ }^{22}$ 
(E)-(4-Ethynylstyryl)(imino)(4-methoxyphenyl)- $\lambda$ 6-sulfanone (5e). The title compound was prepared according to General Procedure D employing vinyl sulfoximine 4 e $(70 \mathrm{mg}, 0.15 \mathrm{mmol})$ and TBAF (1.0 M in THF, $0.16 \mathrm{~mL}, 0.16 \mathrm{mmol})$ in THF (2.8 mL). Purification via flash chromatography (KP-Sil, $0 \%$ grading to $100 \%$ EtOAc/Petroleum ether, eluted at $48 \%$ EtOAc) afforded $\mathbf{5 e}(32 \mathrm{mg}, 72 \%)$ as an orange oil; $\mathrm{R}_{\mathrm{f}}$ $=0.24\left(50 \%\right.$ EtOAc/Petroleum ether); IR (film) $/ \mathrm{cm}^{-1} 3283,3049,2940,2840,1595,1494,1461,1312,1259,1215,1177,1110,976,805 ;{ }^{1} \mathrm{H}$ $\operatorname{NMR}\left(400 \mathrm{MHz}, \mathrm{CDCl}_{3}\right) \delta 7.95-7.93(\mathrm{~d}, J=8.6 \mathrm{~Hz}, 2 \mathrm{H}), 7.52(\mathrm{~d}, J=15.4 \mathrm{~Hz}, 1 \mathrm{H}), 7.46(\mathrm{~d}, J=8.0 \mathrm{~Hz}, 2 \mathrm{H}), 7.39(\mathrm{~d}, J=8.0,2 \mathrm{H}), 6.98(\mathrm{~d}, J=8.7 \mathrm{~Hz}$ $2 \mathrm{H}), 6.90(\mathrm{~d}, J=15.4 \mathrm{~Hz}, 1 \mathrm{H}), 3.85(\mathrm{~s}, 3 \mathrm{H}), 3.18(\mathrm{~s}, 1 \mathrm{H}) ;{ }^{13} \mathrm{C}\left\{{ }^{1} \mathrm{H}\right\} \mathrm{NMR}\left(101 \mathrm{~Hz}, \mathrm{CDCl}_{3}\right) \delta 163.3,139.4,133.8,133.1,132.6(2 \times \mathrm{C}), 131.1,130.2$ $(2 \times \mathrm{C}), 128.2(2 \times \mathrm{C}), 124.4,114.5(2 \times \mathrm{C}), 82.9,79.5,55.7$; HRMS (ESI-TOF) $\mathrm{m} / z$ Calcd for $\mathrm{C}_{17} \mathrm{H}_{16} \mathrm{NO}_{2} \mathrm{~S}^{+}[\mathrm{M}+\mathrm{H}]^{+}, 298.0902$; found, 298.0895 .

(E)-Imino(4-methoxyphenyl)(2-(thiazol-2-yl)vinyl)- $\lambda^{6}$-sulfanone (5f). The title compound was prepared according to General Procedure D employing vinyl sulfoximine $\mathbf{4 F}$ (250 $\mathrm{mg}, 0.48 \mathrm{mmol}$ ) and TBAF (1.0 M in THF, $0.53 \mathrm{~mL}, 0.53 \mathrm{mmol})$ in THF (9 mL). Purification by flash chromatography (KP-Sil, $0 \%$ grading to $100 \%$ EtOAc/Hexane, eluted at $80 \%$ EtOAc) afforded $\mathbf{5 f}(114 \mathrm{mg}, 85 \%)$ as a yellow oil; $\mathrm{R}_{\mathrm{f}}=0.40(80 \%$ EtOAc/Hexane); IR (film)/cm ${ }^{-1} 3259,2926,2840,1593,1494,1312,1258,1231,1126,1108,1090,1021,833 ;{ }^{1} \mathrm{H} \mathrm{NMR}(400 \mathrm{MHz}, \mathrm{CDCl} 3)$ $\delta 7.94(\mathrm{~d}, J=8.94 \mathrm{~Hz}, 2 \mathrm{H}), 7.88(\mathrm{~d}, J=3.2 \mathrm{~Hz}, 1 \mathrm{H}), 7.65(\mathrm{~d}, J=15.2 \mathrm{~Hz}, 1 \mathrm{H}), 7.44(\mathrm{~d}, J=3.2 \mathrm{~Hz}, 1 \mathrm{H}), 7.24(\mathrm{~d}, J=15.3 \mathrm{~Hz}, 1 \mathrm{H}), 6.98(\mathrm{~d}, J=9.0 \mathrm{~Hz}$, 2H), $3.86(\mathrm{~s}, 3 \mathrm{H}), 2.93(\mathrm{bs}, 1 \mathrm{H}) ;{ }^{13} \mathrm{C}\{1 \mathrm{H}\} \mathrm{NMR}\left(101 \mathrm{~Hz}, \mathrm{CDCl}_{3}\right) \delta 163.6,161.4,144.9,135.0,132.8,131.1,130.5(2 \times \mathrm{C}), 127.1,122.1,114.6(2$ $\times$ C), 55.7; HRMS (ESI-TOF) $m / z$ Calcd for $\mathrm{C}_{12} \mathrm{H}_{13} \mathrm{~N}_{2} \mathrm{O}_{2} \mathrm{~S}_{2}+[\mathrm{M}+\mathrm{H}]^{+}, 281.0418$; found, 281.0410 .

(E)-Imino(4-methoxyphenyl)(2-(quinoxalin-2-yl)vinyl)- $\lambda^{6}$-sulfanone (5g). The title compound was prepared according to General Procedure D employing vinyl sulfoximine $\mathbf{4 g}(270 \mathrm{mg}, 0.48 \mathrm{mmol})$ and TBAF (1.0 M in THF, $0.53 \mathrm{~mL}, 0.53 \mathrm{mmol})$ in THF (9.0 mL). Purification by flash chromatography (KP-Sil, $0 \%$ grading to $100 \%$ EtOAc/Hexane, eluted at $90 \%$ EtOAc) afforded $\mathbf{5 g}(120 \mathrm{mg}, 77 \%)$ as a brown oil; $\mathrm{R}_{\mathrm{f}}=$ 0.51 (100\% EtOAc/Hexane); IR (film)/cm-1 3250, 3060, 2840, 1591, 1491, 1413, 1364, 1256, 1230, 1178, 1126, 1013, 969, 827, 760, 670; ${ }^{1} \mathrm{H}$ NMR $\left(400 \mathrm{MHz}_{\mathrm{CDCl}}\right) \delta 8.93(\mathrm{~s}, 1 \mathrm{H}), 8.10-8.07(\mathrm{~m}, 1 \mathrm{H}), 8.04-8.00(\mathrm{~m}, 3 \mathrm{H}), 7.81-7.77(\mathrm{~m}, 3 \mathrm{H}), 7.67(\mathrm{~d}, J=15.2 \mathrm{~Hz}), 7.00-7.03(\mathrm{~m}, 2 \mathrm{H})$, 3.87 (s, 3H), $3.04(\mathrm{bs}, 1 \mathrm{H}) ;{ }^{13} \mathrm{C}\left\{{ }^{1} \mathrm{H}\right\}$ NMR $\left(101 \mathrm{~Hz}, \mathrm{CDCl}_{3}\right) \delta 163.6,146.7,145.0,142.6,142.3,137.8,135.5,132.8,131.0,130.9,130.6(2 \times \mathrm{C})$, 129.7, 129.3, 127.1, $114.6(2 \times \mathrm{C}), 55.7$; HRMS (ESI-TOF) $\mathrm{m} / z$ Calcd for $\mathrm{C}_{17} \mathrm{H}_{17} \mathrm{~N}_{3} \mathrm{O}_{2} \mathrm{~S}^{+}[\mathrm{M}+\mathrm{H}]^{+}, 326.0963$; found, 326.0974 .

Enantioenriched vinyl sulfoximines $(S)-5 h$ and $(R)-5 h$.

$(S)$-Imino(methyl)(p-tolyl)- $\lambda 6$-sulfanone $((\boldsymbol{S})-\mathbf{2 h})$. The title compound was prepared according to General Procedure E employing $(S)-1$ methyl-4-(methylsulfinyl)benzene (S)-6 (500 mg, $3.13 \mathrm{mmol}$, er >99:1). Purification by flash chromatography (KP-Sil, 0\% grading to $100 \%$ EtOAc/Pentane, product eluted at 100\%) afforded $(\boldsymbol{S})-2 \mathbf{h}(473 \mathrm{mg}, 86 \%)$ as a clear oil; $\mathrm{R}_{\mathrm{f}}=0.20(100 \% \mathrm{EtOAc}){ }^{1} \mathrm{H} \mathrm{NMR}(400 \mathrm{MHz}, \mathrm{CDCl} 3)$ $\delta$ 7.90-7.88 (m, 2H), $7.34(\mathrm{~d}, J=8.0 \mathrm{~Hz}, 2 \mathrm{H}), 3.09(\mathrm{~s}, 3 \mathrm{H}), 2.45(\mathrm{~s}, 3 \mathrm{H}) ;{ }^{13} \mathrm{C}\left\{{ }^{1} \mathrm{H}\right\} \mathrm{NMR}\left(101 \mathrm{~Hz}, \mathrm{CDCl}_{3}\right) \delta 144.0,140.5,129.9(2 \times \mathrm{C}), 127.7(2 \times$ C), 46.3, 21.5; HRMS (ESI-TOF) $\mathrm{m} / z$ Calcd $\mathrm{C}_{8} \mathrm{H}_{11} \mathrm{NOS}^{+}[\mathrm{M}+\mathrm{H}]^{+}, 170.0640$; found, 170.0642. Analytical data in agreement with those reported in the literature.31e

$(R)$-Imino(methyl)(p-tolyl)- $\lambda^{6}$-sulfanone $((\boldsymbol{R})-\mathbf{2 h})$. The title compound was prepared according to General Procedure E employing $(R)-1$ methyl-4-(methylsulfinyl) benzene $(250 \mathrm{mg}, 1.62 \mathrm{mmol}$, er >99:1). Purification by flash column chromatography (KP-Sil, $0 \%$ grading to $100 \%$ EtOAc/Pentane, product eluted at 100\%) afforded $(\boldsymbol{R})-2 \mathbf{h}(255 \mathrm{mg}, 94 \%)$ as a clear oil. $\mathrm{R}_{\mathrm{f}}=0.20\left(100 \% \mathrm{EtOAc}^{2} ;{ }^{1} \mathrm{H} \mathrm{NMR}\left(400 \mathrm{MHz}, \mathrm{CDCl}_{3}\right) \delta\right.$ 7.90-7.88 (m, 2H), $7.33(\mathrm{~d}, J=8.0 \mathrm{~Hz}, 2 \mathrm{H}), 3.08(\mathrm{~s}, 3 \mathrm{H}), 2.43(\mathrm{~s}, 3 \mathrm{H}) ;{ }^{13} \mathrm{C}\left\{{ }^{1} \mathrm{H}\right\} \mathrm{NMR}\left(101 \mathrm{~Hz}, \mathrm{CDCl}_{3}\right) \delta 143.9,140.5,129.9(2 \times \mathrm{C}), 127.7(2 \times \mathrm{C})$, 46.4, 21.6; HRMS (ESI-TOF) $\mathrm{m} / z$ Calcd $\mathrm{C}_{8} \mathrm{H}_{11} \mathrm{NOS}^{+}[\mathrm{M}+\mathrm{H}]^{+}, 170.0640$; found, 170.0641. Analytical data in agreement with those reported in the literature. 58

$(S)-(($ tert-Butyldiphenylsilyl)imino)(methyl)( $p$-tolyl)- $\lambda 6$-sulfanone ((S)-3h). The title compound was prepared according to General Procedure B employing $(\boldsymbol{S})-\mathbf{2 h}(531 \mathrm{mg}, 3.14 \mathrm{mmol})$. Purification by flash chromatography (KP-Sil, 0\% grading to 10\% EtOAc/Pentane, product eluted at $10 \%)$ afforded $(\boldsymbol{S})-3 \mathbf{h}(830 \mathrm{mg}, 65 \%,>99: 1 \mathrm{er})$ as a clear oil; $\mathrm{R}_{\mathrm{f}}=0.43(10 \%$ EtOAc/Pentane $) ; \mathrm{IR}(\mathrm{film}) / \mathrm{cm}^{-1} 3049,2955,2855$ $1599,1427,1427,1326,1293,1162,1107,964,820,767,741,700 ;{ }^{1} \mathrm{H} \mathrm{NMR}\left(400 \mathrm{MHz}, \mathrm{CDCl}_{3}\right) \delta 7.82-7.80(\mathrm{~m}, 2 \mathrm{H}), 7.78-7.76(\mathrm{~m}, 2 \mathrm{H}), 7.73-$ $7.70(\mathrm{~m}, 2 \mathrm{H}), 7.41-7.28(\mathrm{~m}, 6 \mathrm{H}), 7.23(\mathrm{~d}, J=8.1 \mathrm{~Hz}, 2 \mathrm{H}), 2.83(\mathrm{~s}, 3 \mathrm{H}), 2.40(\mathrm{~s}, 3 \mathrm{H}), 1.09(\mathrm{~s}, 9 \mathrm{H}) ;{ }^{13} \mathrm{C}\left\{{ }^{1} \mathrm{H}\right\} \mathrm{NMR}(101 \mathrm{~Hz}, \mathrm{CDCl}) \delta 138.2,136.5$, 135.64 $(2 \times \mathrm{C}), 135.58(2 \times \mathrm{C}), 134.8,129.7,129.5(2 \times \mathrm{C}), 129.0(3 \times \mathrm{C}), 127.4,127.0(2 \times \mathrm{C}) 49.1,27.2(9 \times \mathrm{C}), 21.46,19.40 ;$; HRMS $(\mathrm{ESI}-\mathrm{TOF})$ $m / z$ Calcd for $\mathrm{C}_{24} \mathrm{H}_{30} \mathrm{NOSSi}^{+}[\mathrm{M}+\mathrm{H}]^{+}, 408.1817$; found, 408.1834 .

$(R)$-((tert-Butyldiphenylsilyl)imino)(methyl)( $p$-tolyl)- $\lambda 6$-sulfanone $((\boldsymbol{R})-3 \mathbf{h})$. The title compound was prepared according to General Procedure B employing $(\boldsymbol{R})-\mathbf{2 h}(236 \mathrm{mg}, 1.39 \mathrm{mmol})$. Purification by flash column chromatography (KP-Sil, $0 \%$ grading to $10 \%$ EtOAc/Pentane, product eluted at $10 \%)$ afforded $(\boldsymbol{R})-3 \mathbf{h}(346 \mathrm{mg}, 61 \%$, er $>99: 1)$ as a clear oil; $\mathrm{R}_{\mathrm{f}}=0.43(10 \%$ EtOAc/Pentane) IR (film)/(cm-1 3067, 2955, $2855,1590,1472,1427,1323,1293,1162,1107,954,820,768,741,700 ;{ }^{1} \mathrm{H} \mathrm{NMR}(400 \mathrm{MHz}, \mathrm{CDCl} 3) \delta 7.82-7.80(\mathrm{~m}, 2 \mathrm{H}), 7.78-7.76(\mathrm{~m}, 2 \mathrm{H})$, 7.73-7.70 (m, 2H), 7.37-7.29 (m, 6H), $7.23(\mathrm{~d}, J=9.0 \mathrm{~Hz}, 2 \mathrm{H}), 2.83(\mathrm{~s}, 3 \mathrm{H}), 2.40(\mathrm{~s}, 3 \mathrm{H}), 1.09(\mathrm{~s}, 9 \mathrm{H}) ;{ }^{13} \mathrm{C}\left\{{ }^{1} \mathrm{H}\right\} \mathrm{NMR}(101 \mathrm{~Hz}, \mathrm{CDCl} 3) \delta 138.2$, 136.5, $135.6(4 \times \mathrm{C}), 134.8,129.7,129.5(2 \times \mathrm{C}), 129.0(3 \times \mathrm{C}), 127.4,127.0(2 \times \mathrm{C}) 49.1,27.2(9 \times \mathrm{C}), 21.5,19.4 ; \mathrm{HRMS}(\mathrm{ESI}-\mathrm{TOF}) \mathrm{m} / z \mathrm{Calcd}$ for $\mathrm{C}_{24} \mathrm{H}_{30} \mathrm{NOSSi}^{+}[\mathrm{M}+\mathrm{H}]^{+}, 408.1817$; found, 408.1828 .

$(\mathrm{S}, E)-(($ tert-Butyldiphenylsilyl)imino)(4-ethynylstyryl)( $p$-tolyl)- $\lambda 6$-sulfanone $((\boldsymbol{S})-\mathbf{4 h})$. The title compound was prepared according to General Procedure C employing sulfoximine $(\boldsymbol{S})-3 \mathbf{h}(100 \mathrm{mg}, 0.25 \mathrm{mmol}$, er $>99: 1)$ in THF (1.6 mL), $n$ BuLi $(1.3 \mathrm{M}$ in hexane, $0.52 \mathrm{~mL}, 0.68$ $\mathrm{mmol})$, diisopropylamine $(0.05 \mathrm{~mL}, 0.37 \mathrm{mmol})$ in THF $(1.6 \mathrm{~mL})$, diethylchlorophosphate $(0.05 \mathrm{~mL}, 0.32 \mathrm{mmol}), t \mathrm{BuOK}(1.0 \mathrm{M}$ in THF, 0.32 $\mathrm{mL}, 0.32 \mathrm{mmol}$ ), 4-ethynylbenzaldehyde $(85 \mathrm{mg}, 0.47 \mathrm{mmol}$ ) in THF (4 mL). Purification by flash chromatography (KP-Sil, $0 \%$ grading to $10 \%$ EtOAc/Hexane, producr eluted at 8\% EtOAc), afforded $(\boldsymbol{S})-\mathbf{4 h}(88 \mathrm{mg}$, 69\%, er $>99: 1)$ as a yellow oil; Rf $=0.22(10 \%$ EtOAc/Hexane); IR (film)/ $\mathrm{cm}^{-1} 3291,3049,2955,2855,1595,1468,1427,1312,1148,1107,1017,969,797,741,700 ;{ }^{1} \mathrm{H} \mathrm{NMR}(400 \mathrm{MHz}, \mathrm{CDCl} 3) \delta 7.83(\mathrm{~d}$, $J=8.3 \mathrm{~Hz}, 2 \mathrm{H}), 7.79-7.73(\mathrm{~m}, 5 \mathrm{H}), 7.39(\mathrm{~d}, J=8.1 \mathrm{~Hz}, 2 \mathrm{H}), 7.37-7.24(\mathrm{~m}, 8 \mathrm{H}), 7.10(\mathrm{~d}, J=8.3 \mathrm{~Hz}, 2 \mathrm{H}), 6.57(\mathrm{~d}, J=15.3 \mathrm{~Hz}, 1 \mathrm{H}), 3.17(\mathrm{~s}, 1 \mathrm{H})$ $2.40(\mathrm{~s}, 3 \mathrm{H}), 1.14(\mathrm{~s}, 9 \mathrm{H}) ;{ }^{13} \mathrm{C}\left\{{ }^{1} \mathrm{H}\right\}$ NMR $\left(101 \mathrm{MHz}, \mathrm{CDCl}_{3}\right) \delta 142.9,141.2,137.2,136.5,136.3,135.7(2 \times \mathrm{C}), 134.8,133.5,133.2,132.4,129.6$ $(2 \times \mathrm{C}), 129.0,128.1,127.7,127.6,127.4(2 \times \mathrm{C}), 123.8,83.1,79.2,27.2(9 \times \mathrm{C}), 21.5,19.5$; HRMS (ESI-TOF) $\mathrm{m} / z$ Calcd for C $33 \mathrm{H}_{34} \mathrm{NOSSi}^{+}\left[\mathrm{M}^{+}\right.$ $\mathrm{H}]^{+}, 520.2131$; found: 520.2120 .

$(R, E)$-( (tert-Butyldiphenylsilyl)imino)(4-ethynylstyryl)( $p$-tolyl)- $\lambda$-sulfanone $((\boldsymbol{R})-\mathbf{4 h})$. The title compound was prepared according to General Procedure C employing sulfoximine $(\boldsymbol{R})-3 \mathbf{h}(100 \mathrm{mg}, 0.25 \mathrm{mmol}$, er $>99: 1)$ in THF (1.6 mL), $n \mathrm{BuLi}(1.3 \mathrm{M}$ in hexane, $0.52 \mathrm{~mL}, 0.68$ $\mathrm{mmol})$, diisopropylamine $(0.05 \mathrm{~mL}, 0.37 \mathrm{mmol})$ in THF $(1.6 \mathrm{~mL})$, diethylchlorophosphate $(0.05 \mathrm{~mL}, 0.32 \mathrm{mmol}), t \mathrm{BuOK}(1.0 \mathrm{M}$ in THF, 0.32 $\mathrm{mL}, 0.32 \mathrm{mmol}$ ), 4-ethynylbenzaldehyde ( $85 \mathrm{mg}, 0.47 \mathrm{mmol}$ ) in THF (4 mL). Purification by flash column chromatography (KP-Sil, $0 \%$ grading to $10 \%$ EtOAc/Hexane, product eluted at $8 \%$ EtOAc), afforded $(\boldsymbol{R})-\mathbf{4 h}(81 \mathrm{mg}, 62 \%$, er $>99: 1)$ as a yellow oil; $\mathrm{R}_{\mathrm{f}}=0.17(10 \%$ EtOAc/Hexane); IR (film) $/ \mathrm{cm}^{-1} 3291,3049,2955,2855,1681,1554,1472,1427,1312,1151,1107,1017,972,801,741,704 ;{ }^{1} \mathrm{H} \mathrm{NMR}(400 \mathrm{MHz}, \mathrm{CDCl} 3)$ $\delta 7.82(\mathrm{~d}, J=8.3 \mathrm{~Hz}, 2 \mathrm{H}), 7.78-7.72(\mathrm{~m}, 5 \mathrm{H}), 7.38(\mathrm{~d}, J=8.4 \mathrm{~Hz}, 2 \mathrm{H}), 7.33-7.23(\mathrm{~m}, 8 \mathrm{H}), 7.10(\mathrm{~d}, J=8.3 \mathrm{~Hz}, 2 \mathrm{H}), 6.57(\mathrm{~d}, J=15.3 \mathrm{~Hz}, 1 \mathrm{H}), 3.16$ (s, 1H), $2.40(\mathrm{~s}, 3 \mathrm{H}), 1.13(\mathrm{~s}, 9 \mathrm{H}) ;{ }^{13} \mathrm{C}\left\{{ }^{1} \mathrm{H}\right\}$ NMR $\left(101 \mathrm{~Hz}, \mathrm{CDCl}_{3}\right) \delta 142.9,141.2,137.2,136.5,136.3,135.7(2 \times \mathrm{C}), 134.8,133.5,133.2,132.4$,

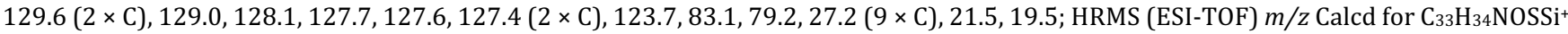
$[\mathrm{M}+\mathrm{H}]^{+}$, 520.2131; found, 520.2130 . 
$(\mathrm{S}, E)$-(4-Ethynylstyryl)(imino)( $p$-tolyl)- $\lambda$-sulfanone $(\boldsymbol{( S )}-\mathbf{5 h})$. The title compound was prepared according to General Procedure D employing sulfoximine $(\boldsymbol{S})-\mathbf{4 h}(80 \mathrm{mg}, 0.15 \mathrm{mmol}$, er $>99: 1)$. Purification by flash column chromatography (KP-Sil, $0 \%$ grading to $100 \%$ EtOAc/Hexane, product eluted at 48\% EtOAc), afforded $(\boldsymbol{S})-5 \mathbf{h}(39 \mathrm{mg}, 92 \%$, er $>99: 1)$ as an off-white solid; Rf $=0.34(50 \%$ EtOAc/Hexane); $\mathrm{mp}\left(\mathrm{CHCl}_{3}\right)=147-149^{\circ} \mathrm{C}$; IR (film)/ $\left.\mathrm{cm}^{-1} 3280,3045,1599,1408,1215,1114,1080,976,861,797,756 ;{ }^{1} \mathrm{H} \mathrm{NMR}_{(400 \mathrm{MHz}, \mathrm{CDCl}}\right) \delta 7.90(\mathrm{~d}$, $J=8.4 \mathrm{~Hz}, 2 \mathrm{H}), 7.56(\mathrm{~d}, J=15.3 \mathrm{~Hz}, 1 \mathrm{H}), 7.47(\mathrm{~d}, J=8.7 \mathrm{~Hz}, 2 \mathrm{H}), 7,41(\mathrm{~d}, J=8.6 \mathrm{~Hz}, 2 \mathrm{H}), 7.32(\mathrm{~d}, J=8.4 \mathrm{~Hz}, 2 \mathrm{H}), 6.93(\mathrm{~d}, J=15.2 \mathrm{~Hz}, 1 \mathrm{H}), 3.19$ (s, 1H), $2.43(\mathrm{~s}, 3 \mathrm{H}), 2.38$ (bs, $1 \mathrm{H}, \mathrm{NH}) ;{ }^{13} \mathrm{C}\left\{{ }^{1} \mathrm{H}\right\} \mathrm{NMR}\left(101 \mathrm{~Hz}, \mathrm{CDCl}_{3}\right) \delta 143.9,139.9,139.5,133.0,132.7(2 \times \mathrm{C}), 130.7,130.0(2 \times \mathrm{C}), 128.3(2$ $\times$ C), $128.0\left(2 \times\right.$ C), 124.4, 82.9, 79.6, 21.56; HRMS (ESI-TOF) $m / z$ Calcd for $\mathrm{C}_{17} \mathrm{H}_{16} \mathrm{NOS}^{+}[\mathrm{M}+\mathrm{H}]^{+}, 282.0953$; found, 282.0952.

$(R, E)$-(4-Ethynylstyryl)(imino)( $p$-tolyl)- $\lambda^{6}$-sulfanone $\left.(\boldsymbol{R})-5 \mathbf{h}\right)$. The title compound was prepared according to General Procedure D employing sulfoximine $(\boldsymbol{R})-\mathbf{4 h}(40 \mathrm{mg}, 0.08 \mathrm{mmol}$, er $>99: 1)$. Purification by flash column chromatography (KP-Sil, $0 \%$ grading to $100 \%$ EtOAc/Hexane, product eluted at 48\% EtOAc), afforded $(\boldsymbol{R})-5 \mathbf{h}(17 \mathrm{mg}, 79 \%$, er $>99: 1)$ as an off-white solid; $\mathrm{R}_{\mathrm{f}}=0.34(50 \% \mathrm{EtOAc} / \mathrm{Hexane})$; $\mathrm{mp}\left(\mathrm{CHCl}_{3}\right)=147-149^{\circ} \mathrm{C} ;{ }^{1} \mathrm{H} \mathrm{NMR}\left(400 \mathrm{MHz} \mathrm{CDCl}_{3}\right) \delta 7.89(\mathrm{~d}, J=8.4 \mathrm{~Hz}, 2 \mathrm{H}), 7.56(\mathrm{~d}, J=15.3 \mathrm{~Hz}, 1 \mathrm{H}), 7.47(\mathrm{~d}, J=8.3 \mathrm{~Hz}, 2 \mathrm{H}), 7.40(\mathrm{~d}, J=8.3$ $\mathrm{Hz}, 2 \mathrm{H}), 7.32(\mathrm{~d}, J=8.3 \mathrm{~Hz}, 2 \mathrm{H}), 6.92(\mathrm{~d}, J=15.2 \mathrm{~Hz}, 1 \mathrm{H}), 3.18(\mathrm{~s}, 1 \mathrm{H}), 2.43(\mathrm{~s}, 3 \mathrm{H}), 2.40(\mathrm{bs}, 1 \mathrm{H}, \mathrm{NH}) ;{ }^{13} \mathrm{C}\left\{{ }^{1} \mathrm{H}\right\} \mathrm{NMR}(101 \mathrm{~Hz}, \mathrm{CDCl} 3) \delta 144.0$, 140.0, $133.0(2 \times \mathrm{C}), 132.7(2 \times \mathrm{C}), 130.7,130.0(2 \times \mathrm{C}), 128.3(2 \times \mathrm{C}), 128.1(2 \times \mathrm{C}), 124.5,82.9,79.6,21.6$; HRMS (ESI-TOF) $\mathrm{m} / z$ Calcd for $\mathrm{C}_{17} \mathrm{H}_{16} \mathrm{NOS}^{+}[\mathrm{M}+\mathrm{H}]^{+}, 282.0953$; found, 282.0952 .

(E)-Trimethyl(2-(phenylthio)vinyl)silane (9a). The title compound was prepared according to General Procedure $\mathrm{F}$ employing $\mathrm{RhCl}\left(\mathrm{PPh}_{3}\right)_{3}(46 \mathrm{mg}, 0.05 \mathrm{mmol})$, ethynyltrimethylsilane $(0.14 \mathrm{~mL}, 1.0 \mathrm{mmol})$ and thiophenol $(102 \mu \mathrm{L}, 1.0 \mathrm{mmol})$. Purification by flash column chromatography (KP-Sil, hexane) afforded vinyl sulfide 9a (179 mg, 86\%) as a colourless oil; $\mathrm{R}_{f}=0.80$ (hexane); IR (film) $/ \mathrm{cm}^{-1} 3061$, $2956,2895,1546,1484,1439,1234,1026,841,742 ;{ }^{1} \mathrm{H}$ NMR $\left(400 \mathrm{MHz}, \mathrm{CDCl}_{3}\right) \delta 7.59-7.01(\mathrm{~m}, 5 \mathrm{H}), 6.59(\mathrm{~d}, J=18.0 \mathrm{~Hz}, 1 \mathrm{H}), 5.82(\mathrm{~d}, J=$ $18.0 \mathrm{~Hz}, 1 \mathrm{H}), 0.00(\mathrm{~s}, 9 \mathrm{H}) ;{ }^{13} \mathrm{C}\left\{{ }^{1} \mathrm{H}\right\} \mathrm{NMR}\left(101 \mathrm{~Hz}, \mathrm{CDCl}_{3}\right) \delta 145.0,135.2,129.4(2 \times \mathrm{C}), 129.1(2 \times \mathrm{C}), 125.6,122.8,-0.8(9 \times \mathrm{C}) ; \mathrm{HRMS}\left(\mathrm{APCI}{ }^{+}\right)$ $\mathrm{m} / \mathrm{z}$ : Calcd for $\mathrm{C}_{11} \mathrm{H}_{17} \mathrm{SSi}^{+}[\mathrm{M}+\mathrm{H}]^{+}: 209.0815$; found: 209.0817 . Analytical data in agreement with those reported in the literature.59

$(E)$-(2-(Benzylthio)vinyl)trimethylsilane (9b). The title compound was prepared according to General Procedure $\mathrm{F}$ employing $\mathrm{RhCl}\left(\mathrm{PPh}_{3}\right)_{3}(92 \mathrm{mg}, 0.10 \mathrm{mmol})$, ethynyltrimethylsilane $(0.28 \mathrm{~mL}, 2.0 \mathrm{mmol})$ and benzyl mercaptan $(0.23 \mathrm{~mL}, 2.0 \mathrm{mmol})$. Purification by flash column chromatography $\left(\mathrm{SiO}_{2}\right.$, hexane) afforded vinyl sulfide $9 \mathrm{~b}$ (356 mg, 80\%) as a colourless oil; $\mathrm{R}_{f}=0.60$ (hexane); IR (film) $/ \mathrm{cm}^{-1}$ $\left.3060,3027,2960,2922,2859,1603,1548,1495,1454,1416,1374,1334,1200,1103,1070,917,850,768,701 ;{ }^{1} \mathrm{H} \mathrm{NMR}_{(400} \mathrm{MHz}_{\mathrm{C}} \mathrm{CDCl}\right)$ $\delta 7.38-7.27(\mathrm{~m}, 5 \mathrm{H}), 6.55(\mathrm{~d}, J=18.1 \mathrm{~Hz}, 1 \mathrm{H}), 5.79(\mathrm{~d}, J=18.1 \mathrm{~Hz}, 1 \mathrm{H}), 3.95(\mathrm{~s}, 2 \mathrm{H}), 0.06(\mathrm{~s}, 9 \mathrm{H}) ;{ }^{13} \mathrm{C}\left\{{ }^{1} \mathrm{H}\right\} \mathrm{NMR}(101 \mathrm{~Hz}, \mathrm{CDCl} 3) \delta 138.3,137.3$,

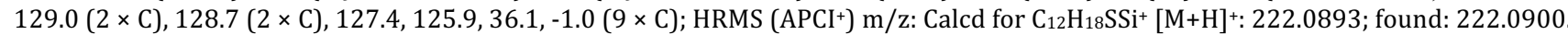

(E)-(4-Methoxystyryl)(naphthalen-2-yl)sulfane (9c). The title compound was prepared according to General Procedure $\mathrm{F}$ employing $\mathrm{RhCl}\left(\mathrm{PPh}_{3}\right)_{3}(46 \mathrm{mg}, 0.05 \mathrm{mmol})$, naphthalene-2-thiol (176 mg, $\left.1.1 \mathrm{mmol}\right)$ and 1-ethynyl-4-methoxybenzene $(129 \mu \mathrm{L}, 1.0 \mathrm{mmol}) . \mathrm{Purification}$ by flash column chromatography $\left(\mathrm{SiO}_{2}, 5 \% \mathrm{Et}_{2} \mathrm{O} /\right.$ hexane $)$ afforded vinyl sulfide $9 \mathrm{c}(227 \mathrm{mg}, 78 \%)$ as a white solid; $\mathrm{R}_{f}=0.40\left(5 \% \mathrm{Et}_{2} \mathrm{O} / \mathrm{hex}_{-}\right.$ ane); $\mathrm{mp}=103-105^{\circ} \mathrm{C}$; IR (film) $/ \mathrm{cm}^{-1} 3056,3004,1602,1509,1252,1032,950,846,801 ;{ }^{1} \mathrm{H} \mathrm{NMR}(400 \mathrm{MHz}, \mathrm{CDCl} 3) \delta 7.87-7.75(\mathrm{~m}, 4 \mathrm{H})$, 7.53-7.44 (m, 3H), 7.38-7.32 (m, 2H), 6.92-6.85 (m, 2H), $6.83(\mathrm{~s}, 2 \mathrm{H}), 3.83(\mathrm{~s}, 3 \mathrm{H}) ;{ }^{13} \mathrm{C}\left\{{ }^{1} \mathrm{H}\right\} \mathrm{NMR}\left(101 \mathrm{~Hz}, \mathrm{CDCl}_{3}\right) \delta 159.5,133.8,133.4,133.1$, $132.1,129.4,128.6,127.8,127.5(2 \times \mathrm{C}), 127.4,127.2(2 \times \mathrm{C}), 126.6,125.9,119.8,114.2(2 \times \mathrm{C}), 55.3 ; \mathrm{HRMS}_{\left(\mathrm{APCI}^{+}\right)}{\mathrm{m} / \mathrm{z} \mathrm{Calcd} \text { for C }}_{19} \mathrm{H}_{17} \mathrm{OS}{ }^{+}$ $[\mathrm{M}+\mathrm{H}]^{+}:$374.0420; Found: 374.0437 . Analytical data in agreement with those reported in the literature. 60

$(E)$-(4-Bromophenyl)(styryl)sulfane (9d). The title compound was prepared according to General Procedure $\mathrm{F}$ employing $\left.\mathrm{RhCl}_{(\mathrm{PPh}}\right)_{3}$ (92 $\mathrm{mg}, 0.1 \mathrm{mmol}$ ), 4-bromobenzenethiol (378 mg, $2.0 \mathrm{mmol}$ ) and ethynylbenzene $(0.22 \mathrm{~mL}, 2.0 \mathrm{mmol}$. Purification by flash column chromatography $\left(\mathrm{SiO}_{2}\right.$, pentane) afforded vinyl sulfide $9 \mathrm{~b}(576 \mathrm{mg}, 99 \%)$ as an off-white solid; $\mathrm{R}_{f}=0.45$ (pentane); $\mathrm{mp}=77-78{ }^{\circ} \mathrm{C}$; IR (film) $/ \mathrm{cm}^{-}$ $\left.13079,3056,3019,2933,1897,1774,1737,1664,1599,1472,1387,1230,1088,1032,989,954,813,742,693 ;{ }^{1} \mathrm{H} \mathrm{NMR}^{(400} \mathrm{MHz} \mathrm{CDCl}_{3}\right)$ $\delta$ 7.50-7.42 (m, 2H), 7.39-7.19 (m, 7H), 6.87-6.72 (m, 2H); ${ }^{3} \mathrm{C}\left\{{ }^{1} \mathrm{H}\right\} \mathrm{NMR}\left(101 \mathrm{~Hz}, \mathrm{CDCl}_{3}\right) \delta 136.3,134.7,133.1,132.3(2 \times \mathrm{C}), 131.1(2 \times \mathrm{C})$, $128.8(2 \times \mathrm{C}), 128.0,126.2(2 \times \mathrm{C}), 122.3,120.9$; HRMS $\left(+\mathrm{p}\right.$ APCI) $\mathrm{m} / \mathrm{z}$ : Calcd for $\mathrm{C}_{14} \mathrm{H}_{12} \mathrm{SBr}^{+}[\mathrm{M}+\mathrm{H}]^{+}: 290.0938$; Found: 290.9830.

Butyl (E)-3-((4-bromostyryl)thio)propanoate (9e). The title compound was prepared according to an adaptation of General Procedure $\mathrm{F}$, including heating to $65^{\circ} \mathrm{C}$ and increasing the catalyst loading to $10 \mathrm{~mol} \%$. Employing $\left.\mathrm{RhCl}_{(\mathrm{PPh}}\right)_{3}(92 \mathrm{mg}, 0.1 \mathrm{mmol}), 1-\mathrm{bromo}-4$ ethynylbenzene ( $181 \mathrm{mg}, 1.0 \mathrm{mmol})$, butyl 3-mercaptopropanoate $(178 \mu \mathrm{L}, 1.1 \mathrm{mmol})$. Purification by flash column chromatography ( $\mathrm{SiO}_{2}$ $5 \% \mathrm{Et}_{2} \mathrm{O} /$ hexane) afforded vinyl sulfide $9 \mathrm{e}(185 \mathrm{mg}, 53 \%)$ as a white solid; $\mathrm{R}_{f}=0.14\left(5 \% \mathrm{Et}_{2} \mathrm{O} / \mathrm{hexane}\right) ; \mathrm{mp}=55-57^{\circ} \mathrm{C} ; \mathrm{IR}(\mathrm{film}) / \mathrm{cm}^{-1} 2959$, $2870,1722,1595,1487,1401,1364,1241,1178,1107,939,827 ;{ }^{1}{ }^{H}$ NMR $\left(400 \mathrm{MHz}, \mathrm{CDCl}_{3}\right) \delta 7.45-7.37(\mathrm{~m}, 2 \mathrm{H}), 7.19-7.11(\mathrm{~m}, 2 \mathrm{H}), 6.71(\mathrm{~d}$, $J=15.5 \mathrm{~Hz}, 1 \mathrm{H}), 6.43(\mathrm{~d}, J=15.6 \mathrm{~Hz}, 1 \mathrm{H}), 4.11(\mathrm{t}, J=6.7 \mathrm{~Hz}, 2 \mathrm{H}), 3.07(\mathrm{t}, J=7.3 \mathrm{~Hz}, 2 \mathrm{H}), 2.71(\mathrm{t}, J=7.3 \mathrm{~Hz}, 2 \mathrm{H}), 1.66-1.58(\mathrm{~m}, 2 \mathrm{H}), 1.46-1.31$ $(\mathrm{m}, 2 \mathrm{H}), 0.93(\mathrm{t}, J=7.4 \mathrm{~Hz}, 3 \mathrm{H}) ;{ }^{13} \mathrm{C}\left\{{ }^{1} \mathrm{H}\right\} \mathrm{NMR}\left(101 \mathrm{~Hz}, \mathrm{CDCl}_{3}\right) \delta 171.6,135.7,131.7(2 \times \mathrm{C}), 127.0(2 \times \mathrm{C}), 126.6,125.1,120.7,64.8,34.6,30.6$, 27.6, 19.1, 13.7; HRMS (APCI+ $\mathrm{m} / \mathrm{z}$ : Calcd for $\mathrm{C}_{15} \mathrm{H}_{20} \mathrm{O}_{2} \mathrm{SBr}^{+}[\mathrm{M}+\mathrm{H}]^{+}: 343.0362$; Found: 343.037 .

tert-Butyl (S,E)-(tert-butoxycarbonyl)(5-phenyl-1-(phenylthio)pent-1-en-3-yl)carbamate (9f). The title compound was prepared according to an adaptation of General Procedure F, increasing the reaction time to $72 \mathrm{~h}$ and employing $\mathrm{RhCl}\left(\mathrm{PPh}_{3}\right)_{3}(73 \mathrm{mg}, 0.08 \mathrm{mmol})$, tert-butyl (S)-(tert-butoxycarbonyl)(5-phenylpent-1-yn-3-yl)carbamate13e $(950 \mathrm{mg}, 2.65 \mathrm{mmol})$, thiophenol (301 $\mu \mathrm{L}, 2.94 \mathrm{mmol})$. Purification by flash column chromatography $\left(\mathrm{SiO}_{2}, 5 \%\right.$ EtOAc/hexane) afforded vinyl sulfide $\mathbf{9 f}\left(1.22 \mathrm{~g}\right.$, 98\%) as a colouless oil; $\mathrm{R}_{f}=0.75$ (10\% EtOAc/hexane); IR (film) $/ \mathrm{cm}^{-1}$ 2978, 1737, 1799, 1342, 1244, 1140, 1114; ${ }^{1} \mathrm{H}$ NMR $\left(400 \mathrm{MHz}, \mathrm{CDCl}_{3}\right) \delta \delta 7.35-7.27$ (m, 5H), 7.26-7.13 (m, 5H), 6.36 (dd, J= $15.2,0.9 \mathrm{~Hz}, 1 \mathrm{H}), 6.06(\mathrm{dd}, J=15.2,7.6 \mathrm{~Hz}, 1 \mathrm{H}), 4.76(\mathrm{q}, J=7.4 \mathrm{~Hz}, 1 \mathrm{H}), 2.62(\mathrm{t}, J=8.1 \mathrm{~Hz}, 2 \mathrm{H}), 2.29-2.14(\mathrm{~m}, 1 \mathrm{H}), 2.14-1.95(\mathrm{~m}, 1 \mathrm{H}), 1.49(\mathrm{~s}$, $18 \mathrm{H})$. Analytical data in agreement with those reported in the literature. Error! Bookmark not defined.

(E)-Imino(phenyl)(2-(trimethylsilyl)vinyl)- $\lambda$-sulfanone (10a). The title compound was prepared according to General Procedure A employing vinyl sulfide $9 \mathrm{a}$ ( $179 \mathrm{mg}, 0.86 \mathrm{mmol}$ ). Purification by flash column chromatography (KP-Sil, $0 \%$ grading to $100 \%$ EtOAc/hexane, product eluted at $38 \%$ EtOAc) afforded sulfoximine 10 a $(107 \mathrm{mg}, 52 \%)$ as a colourless oil; $\mathrm{R}_{f}=0.40\left(50 \%\right.$ EtOAc/hexane); IR (film) $/ \mathrm{cm}^{-1}$ $2957,2905,1504,1450,1266,1213,978,841,789 ;{ }^{1} \mathrm{H} \mathrm{NMR}\left(400 \mathrm{MHz}, \mathrm{CDCl}_{3}\right) \delta 8.02-7.89(\mathrm{~m}, 2 \mathrm{H}), 7.64-7.43(\mathrm{~m}, 3 \mathrm{H}), 7.15(\mathrm{~d}, J=17.6 \mathrm{~Hz}$, $1 \mathrm{H}), 6.69(\mathrm{~d}, J=17.6 \mathrm{~Hz}, 1 \mathrm{H}), 0.11(\mathrm{~s}, 9 \mathrm{H}) ;{ }^{13} \mathrm{C}\left\{{ }^{1} \mathrm{H}\right\} \mathrm{NMR}\left(101 \mathrm{~Hz}, \mathrm{CDCl}_{3}\right) \delta 143.9,143.4,142.2,132.9,129.3(2 \times \mathrm{C}), 128.3(2 \times \mathrm{C}),-1.8(9 \times \mathrm{C})$; HRMS (ESI-TOF) m/z: Calcd for $\mathrm{C}_{11} \mathrm{H}_{18} \mathrm{NOSSi}^{+}[\mathrm{M}+\mathrm{H}]^{+}: 240.0873$; Found: 240.0871.

$(E)$-Benzyl(imino)(2-(trimethylsilyl)vinyl)- $\lambda{ }^{6}$-sulfanone (10b). The title compound was prepared according to General Procedure A employing vinyl sulfide $\mathbf{9 b}(205 \mathrm{mg}, 0.92 \mathrm{mmol})$. Purification by flash column chromatography $\left(\mathrm{SiO}_{2}, 50 \%\right.$ EtOAc/hexane) afforded sulfoximine $\mathbf{1 0 b}(178 \mathrm{mg}, 76 \%)$ as a colourless oil; $\mathrm{R}_{f}=0.34$ (50\% EtOAc/hexane); IR (film)/cm ${ }^{-1} 2955,1494,1453,1248,1215,976,842 ;{ }^{1} \mathrm{H} \mathrm{NMR}(400$ $\left.\mathrm{MHz}_{\mathrm{CDCl}}\right) \delta 7.42-7.32(\mathrm{~m}, 5 \mathrm{H}), 7.05(\mathrm{~d}, J=17.8 \mathrm{~Hz}, 1 \mathrm{H}), 6.62(\mathrm{~d}, J=17.8 \mathrm{~Hz}, 1 \mathrm{H}), 4.30(\mathrm{~d}, J=13.2 \mathrm{~Hz}, 1 \mathrm{H}), 4.20(\mathrm{~d}, J=13.2 \mathrm{~Hz}, 1 \mathrm{H}), 2.63(\mathrm{~s}$, $1 \mathrm{H}), 0.14(\mathrm{~s}, 9 \mathrm{H}) ;{ }^{13} \mathrm{C}\left\{{ }^{1} \mathrm{H}\right\} \operatorname{NMR}\left(101 \mathrm{~Hz}, \mathrm{CDCl}_{3}\right) \delta 148.0,140.7,130.9,128.6,128.5(2 \times \mathrm{C}), 128.3,62.7,-2.1(9 \times \mathrm{C}) ; \mathrm{HRMS}(\mathrm{ESI}-\mathrm{TOF}) \mathrm{m} / \mathrm{z}$ : Calcd for $\mathrm{C}_{12} \mathrm{H}_{20} \mathrm{NOSSi}^{+}[\mathrm{M}+\mathrm{H}]+:$ : 254.1035; Found: 254.1027.

(E)-Imino(4-methoxystyryl)(naphthalen-2-yl)- $\lambda 6$-sulfanone (10c). The title compound was prepared according to General Procedure A employing vinyl sulfide 9c (197 mg, $0.67 \mathrm{mmol})$. Purification by flash column chromatography ( $\mathrm{SiO}_{2}, 50 \%$ EtOAc/hexane) afforded sulfoximine 10c (145 mg, 67\%) as a colourless oil; $\mathrm{R}_{f}=0.24$ (50\% EtOAc/hexane); IR (film)/cm-1 3268, 3056, 2840, 1602, 1509, 1256, 1215, 
1174, 1066, 976, 864, 813, 757; ${ }^{1} \mathrm{H}$ NMR $\left(400 \mathrm{MHz}^{\mathrm{C} C D C l}\right)_{3} \delta 8.64-8.60(\mathrm{~m}, 1 \mathrm{H}), 8.02-7.94(\mathrm{~m}, 3 \mathrm{H}), 7.92-7.89(\mathrm{~m}, 1 \mathrm{H}), 7.67-7.59(\mathrm{~m}, 3 \mathrm{H})$, 7.46-7.41 (m, 2H), 6.91-6.83 (m, 3H), $3.83(\mathrm{~s}, 3 \mathrm{H}), 3.01(\mathrm{~s}, 1 \mathrm{H}) ;{ }^{13} \mathrm{C}\left\{{ }^{1} \mathrm{H}\right\} \mathrm{NMR}\left(101 \mathrm{~Hz}, \mathrm{CDCl}_{3}\right) \delta 161.8,141.5,140.1,134.8,132.4,130.2(2 \times \mathrm{C})$, 129.4, 129.3, 128.9, 128.8, 127.8, 127.4, 126.5, 125.3, 123.1, $\left.114.4(2 \times \mathrm{C}), 55.4 ; \mathrm{HRMS}_{(\mathrm{APCI}+}\right) \mathrm{m} / \mathrm{z}$ : Calcd for $\mathrm{C}_{19} \mathrm{H}_{18} \mathrm{NO}_{2} \mathrm{~S}^{+}[\mathrm{M}+\mathrm{H}]+: 324.1053$; Found: 324.1046 .

(E)-(4-Bromophenyl)(imino)(styryl)- $\lambda^{6}$-sulfanone (10d). The title compound was prepared according to General Procedure A employing vinyl sulfide 9d (291 mg, $1.0 \mathrm{mmol})$. Purification by flash column chromatography $\left(\mathrm{SiO}_{2}, 30 \%\right.$ EtOAc/hexane) afforded sulfoximine $\mathbf{1 0 d}$ (263 mg, 82\%) as a yellow oil; $\mathrm{R}_{f}=0.38$ (30\% EtOAc/hexane); IR (film)/ $\mathrm{cm}^{-1} 3257,3056,1729,1613,1572,1464,1386,1237,965,790 ;{ }^{1} \mathrm{H}$ NMR $\left(400 \mathrm{MHz}, \mathrm{CDCl}_{3}\right) \delta 7.94-7.85(\mathrm{~m}, 2 \mathrm{H}), 7.70-7.60(\mathrm{~m}, 3 \mathrm{H}), 7.51-7.45(\mathrm{~m}, 2 \mathrm{H}), 7.44-7.34(\mathrm{~m}, 3 \mathrm{H}), 6.91(\mathrm{dd}, J=15.2,1.0 \mathrm{~Hz}, 1 \mathrm{H}), 2.98(\mathrm{~s}$, $1 \mathrm{H}) ;{ }^{13} \mathrm{C}\left\{{ }^{1} \mathrm{H}\right\}$ NMR $\left(101 \mathrm{~Hz}, \mathrm{CDCl}_{3}\right) \delta 142.1,142.0,132.4(3 \times \mathrm{C}), 131.0,129.5(2 \times \mathrm{C}), 129.0(2 \times \mathrm{C}), 129.0,128.5(2 \times \mathrm{C}), 128.0 ; \mathrm{HRMS}\left(\mathrm{APCI}{ }^{+}\right)$ $\mathrm{m} / \mathrm{z}$ : Calcd for $\mathrm{C}_{14} \mathrm{H}_{13} \mathrm{NOSBr}^{+}[\mathrm{M}+\mathrm{H}]^{+}:$321.9901; Found: 321.9895 .

Butyl (E)-3-(2-(4-bromophenyl)vinylsulfonimidoyl)propanoate (10e). The title compound was prepared according to General Procedure A employing vinyl sulfide $9 e(96 \mathrm{mg}, 0.28 \mathrm{mmol})$. Purification by flash column chromatography $\left(\mathrm{SiO}_{2}, 50 \%\right.$ EtOAc/hexane) afforded sulfoximine 10e (84 mg, 80\%) as a white solid; $\mathrm{R}_{f}=0.23$ (50\% EtOAc/hexane); $\mathrm{mp}=57-59{ }^{\circ} \mathrm{C}$; IR (film) $/ \mathrm{cm}^{-1} 3280,2959,2870,1729,1617$, $1487,1244,1211,1185,1069,1006,857,801 ;{ }^{1} \mathrm{H}$ NMR $\left(400 \mathrm{MHz}, \mathrm{CDCl}_{3}\right) \delta 7.59-7.52(\mathrm{~m}, 2 \mathrm{H}), 7.47(\mathrm{~d}, J=15.3 \mathrm{~Hz}, 1 \mathrm{H}), 7.41-7.33(\mathrm{~m}, 2 \mathrm{H})$, $6.89(\mathrm{~d}, J=15.3 \mathrm{~Hz}, 1 \mathrm{H}), 4.04(\mathrm{t}, J=6.7 \mathrm{~Hz}, 2 \mathrm{H}), 3.46(\mathrm{td}, J=7.4,1.7 \mathrm{~Hz}, 2 \mathrm{H}), 2.84(\mathrm{td}, J=7.2,2.2 \mathrm{~Hz}, 2 \mathrm{H}), 2.73(\mathrm{~s}, 1 \mathrm{H}), 1.61-1.48(\mathrm{~m}, 2 \mathrm{H}), 1.39-$ $1.24(\mathrm{~m}, 2 \mathrm{H}), 0.89(\mathrm{t}, J=7.4 \mathrm{~Hz}, 3 \mathrm{H}) ;{ }^{13} \mathrm{C}\left\{{ }^{1} \mathrm{H}\right\} \operatorname{NMR}\left(101 \mathrm{~Hz}, \mathrm{CDCl}_{3}\right) \delta 170.5,142.6,132.4(2 \times \mathrm{C}), 131.3,129.8(2 \times \mathrm{C}), 128.1,125.6,65.2,51.6$, 30.4, 28.4, 19.0, 13.6; HRMS (APCI+) m/z: Calcd for $\mathrm{C}_{15} \mathrm{H}_{21} \mathrm{NO}_{3} \mathrm{SBr}^{+}[\mathrm{M}+\mathrm{H}]^{+}:$393.0995; Found: 393.1003.

tert-Butyl (tert-butoxycarbonyl)((3S,E)-5-phenyl-1-(phenylsulfonimidoyl)pent-1-en-3-yl)carbamate (10f). The title compound was prepared according to General Procedure A employing vinyl sulfide $9 f(87 \mathrm{mg}, 0.18 \mathrm{mmol}$ ). Purification by flash column chromatography (KPSil, $0 \%$ grading to $10 \% \mathrm{MeOH} / \mathrm{CHCl}_{3}$, eluted at $\left.8 \% \mathrm{MeOH}\right)$ afforded sulfoximine $\mathbf{1 0 f}(55 \mathrm{mg}, 91 \%)$ as a colouless oil in a $1: 1 \mathrm{mixture}$ of diastereoisomers; $\mathrm{R}_{f}=0.60\left(5 \% \mathrm{MeOH} / \mathrm{CHCl}_{3}\right)$; IR (film)/cm $\left.{ }^{-1} 2978,1740,1703,1368,1233,1140 ;{ }^{1} \mathrm{H} \mathrm{NMR}_{(400} \mathrm{MHz} \mathrm{CDCl}_{3}\right) \delta 8.02-7.93$ $(\mathrm{m}, 2 \mathrm{H}), 7.63-7.48(\mathrm{~m}, 3 \mathrm{H}), 7.25-7.11(\mathrm{~m}, 5 \mathrm{H}), 7.05(\mathrm{dd}, J=15.1,5.1 \mathrm{~Hz}, 1 \mathrm{H}), 6.45(\mathrm{dd}, J=15.1,2.9 \mathrm{~Hz}, 1 \mathrm{H}), 5.02-4.89(\mathrm{~m}, 1 \mathrm{H}), 2.88(\mathrm{~s}, 1 \mathrm{H})$, 2.70-2.55 (m, 2H), 2.37-2.20 (m, 1H), 2.16-2.00 (m, 1H), $1.45(\mathrm{~s}, 9 \mathrm{H}), 1.45(\mathrm{~s}, 9 \mathrm{H}) ;{ }^{13} \mathrm{C}\left\{{ }^{1} \mathrm{H}\right\} \mathrm{NMR}\left(101 \mathrm{~Hz}, \mathrm{CDCl}_{3}\right) \delta 152.3(2 \times \mathrm{C}), 144.6,144.6$, $142.5,140.8,132.9(2 \times \mathrm{C}), 129.3(2 \times \mathrm{C}), 128.6(2 \times \mathrm{C}), 128.4(2 \times \mathrm{C}), 128.0(2 \times \mathrm{C}), 126.3,83.2(2 \times \mathrm{C}), 55.8,34.0,33.8,32.4,28.0(6 \times \mathrm{C})$ HRMS (ESI-TOF) m/z: Calcd for $\mathrm{C}_{27} \mathrm{H}_{37} \mathrm{~N}_{2} \mathrm{O}_{5} \mathrm{~S}^{+}[\mathrm{M}+\mathrm{H}]+$ : 501.2423 ; Found: 501.2434 .

$((S, E)$-3-Amino-5-phenylpent-1-en-1-yl)(imino)(phenyl)- $\lambda 6$-sulfanone $(\mathbf{1 0 g})$. Trifluoroacetic acid $(1.1 \mathrm{~mL})$ was added dropwise to a solution of sulfoximine $10 \mathrm{f}(194 \mathrm{mg}, 0.39 \mathrm{mmol})$ in $\mathrm{CH}_{2} \mathrm{Cl}_{2}(3.8 \mathrm{~mL})$ at $0{ }^{\circ} \mathrm{C}$ and the reaction stirred for $3 \mathrm{~h}$. The solvent was then removed under reduced pressure. The crude was dissolved in $20 \mathrm{~mL} \mathrm{CH} \mathrm{Cl}_{2}$ and washed with sat. aq. $\mathrm{NaHCO}_{3}(3 \times 20 \mathrm{~mL})$. The solvent was removed under reduced pressure and purification by flash column chromatography (KP-Sil, $0 \%$ grading to $10 \% \mathrm{MeOH} / \mathrm{CHCl}_{3}$, eluted at $5 \% \mathrm{MeOH}$ ) afforded sulfoximine $\mathbf{1 0 g}$ (89 mg, 76\%) as a yellow oil in a $1: 1$ mixture of diastereoisomers; $\mathrm{R}_{f}=0.50\left(10 \% \mathrm{MeOH} / \mathrm{CH}_{2} \mathrm{Cl} 2\right) ; \mathrm{IR}$ (film)/ $\mathrm{cm}^{-1}$ $3273,3027,2922,1602,1446,1222,1088,969,749,685 ;{ }^{1} \mathrm{H}$ NMR $\left(400 \mathrm{MHz}, \mathrm{CDCl}_{3}\right) \delta 7.95(\mathrm{~d}, J=7.2 \mathrm{~Hz}, 2 \mathrm{H}), 7.60-7.47(\mathrm{~m}, 3 \mathrm{H}), 7.29-7.22$ $(\mathrm{m}, 2 \mathrm{H}), 7.20-7.08(\mathrm{~m}, 3 \mathrm{H}), 6.90(\mathrm{dd}, J=14.8,5.8 \mathrm{~Hz}, 1 \mathrm{H}), 6.53(\mathrm{~d}, J=14.9 \mathrm{~Hz}, 1 \mathrm{H}), 3.55-3.44(\mathrm{~m}, 1 \mathrm{H}), 2.70-2.61(\mathrm{~m}, 2 \mathrm{H}), 2.44(\mathrm{~s}, 1 \mathrm{H}), 1.90-$ $1.70(\mathrm{~m}, 2 \mathrm{H}), 1.39(\mathrm{~s}, 2 \mathrm{H}) ;{ }^{13} \mathrm{C}\left\{{ }^{1} \mathrm{H}\right\} \operatorname{NMR}\left(101 \mathrm{~Hz}, \mathrm{CDCl}_{3}\right) \delta 148.9,148.8,142.9,141.1,141.1,132.9,131.7,129.3(2 \times \mathrm{C}), 128.6(2 \times \mathrm{C}), 128.4$ $(2 \times \mathrm{C}), 128.0(2 \times \mathrm{C}), 126.2,51.6,38.4,38.3,32.1$; HRMS $\left(\mathrm{APCI}^{+}\right) \mathrm{m} / \mathrm{z}$ : Calcd for $\mathrm{C}_{17} \mathrm{H}_{21} \mathrm{~N}_{2} \mathrm{OS}^{+}[\mathrm{M}+\mathrm{H}]^{+}$: 301.1369 ; Found: 301.1377 .

(2-Hydroxyethyl)(imino)(phenyl)- $\lambda^{6}$-sulfanone (12a). The title compound was prepared according to General Procedure A employing sulfide $11 \mathrm{a}(135 \mu \mathrm{L}, 1.0 \mathrm{mmol})$ in $\mathrm{MeOH}(2.0 \mathrm{~mL})$ at $25^{\circ} \mathrm{C}$. After $3 \mathrm{~h}$ the solvent was removed under reduced pressure. Purification by flash column chromatography $\left(\mathrm{SiO}_{2}, 50 \%\right.$ acetone/pentane) afforded sulfoximine 12a (136 mg, 73\%) as a colourless oil; $\mathrm{R}_{f}=0.29(50 \%$ acetone/pentane); IR (film)/ $\mathrm{cm}^{-1} 3261,1446,1215,1095,1059,987,760,723,699 ;{ }^{1} \mathrm{H}$ NMR $\left(400 \mathrm{MHz}, \mathrm{CDCl}_{3}\right) \delta 8.04-7.98$ (m, $\left.2 \mathrm{H}\right), 7.71-7.64$ $(\mathrm{m}, 1 \mathrm{H}), 7.64-7.57(\mathrm{~m}, 2 \mathrm{H}), 4.70(\mathrm{t}, J=6.1 \mathrm{~Hz}, 1 \mathrm{H}), 4.08(\mathrm{ddd}, J=12.6,6.5,3.8 \mathrm{~Hz}, 1 \mathrm{H}), 3.90-3.78(\mathrm{~m}, 1 \mathrm{H}), 3.32(\mathrm{dt}, J=6.1,2.9 \mathrm{~Hz}, 2 \mathrm{H}), 2.88$ $(\mathrm{s}, 1 \mathrm{H}) ;{ }^{13} \mathrm{C}\left\{{ }^{1} \mathrm{H}\right\}$ NMR $\left(101 \mathrm{~Hz}, \mathrm{CDCl}_{3}\right) \delta 141.8,133.6,129.4(2 \times \mathrm{C}), 128.3(2 \times \mathrm{C}), 58.2,56.4$; Analytical data in agreement with those reported in the literature. $31 \mathrm{~b}$

2-Hydroxyethyl(imino)(4-nitrophenyl)- $\lambda^{6}$-sulfanone (12b). The title compound was prepared according to General Procedure A employing sulfide 11b (199 mg, 1.0 mmol. Purification by flash column chromatography $\left(\mathrm{SiO}_{2}, 1-5 \% \mathrm{MeOH} / \mathrm{CH}_{2} \mathrm{Cl}_{2}\right)$ afforded sulfoximine $\mathbf{1 2 b}$ (201 mg, 87\%) as a white solid; $\mathrm{R}_{\mathrm{f}}=0.15\left(4 \% \mathrm{MeOH} / \mathrm{CH}_{2} \mathrm{Cl}_{2}\right) ; \mathrm{mp}=131-133^{\circ} \mathrm{C}$; IR (film) $/ \mathrm{cm}^{-1} 3571,3381,3153,3104,2960,1633,1607$, $1521,1469,1431,1349,1308,1215,1141,1088,1029,992,947,857,731 ;{ }^{1} \mathrm{H}$ NMR $\left(400 \mathrm{MHz}, \mathrm{DMSO}-\mathrm{d}_{6}\right) \delta 8.39(\mathrm{~d}, J=8.9 \mathrm{~Hz}, 2 \mathrm{H}), 8.15(\mathrm{~d}, J$ $=8.9 \mathrm{~Hz}, 2 \mathrm{H}), 3.68(\mathrm{t}, J=6.2 \mathrm{~Hz}, 2 \mathrm{H}), 3.41(\mathrm{t}, J=6.2 \mathrm{~Hz}, 2 \mathrm{H}) ;{ }^{13} \mathrm{C}\left\{{ }^{1} \mathrm{H}\right\} \mathrm{NMR}\left(101 \mathrm{~Hz}, \mathrm{DMSO}-\mathrm{d}_{6}\right) \delta 149.9,148.6,129.9(2 \times \mathrm{C}), 124.1(2 \times \mathrm{C}), 58.8$, 55.7; HRMS (APCI+) m/z: Calcd for $\mathrm{C}_{8} \mathrm{H}_{11} \mathrm{~N}_{2} \mathrm{O}_{4} \mathrm{~S}^{+}[\mathrm{M}+\mathrm{H}]^{+}: 231.0434$; Found: 231.0433.

Benzo[d]thiazol-2-yl(2-hydroxyethyl)(imino)- $\lambda^{6}$-sulfanone (12c). The title compound was prepared according to General Procedure A employing sulfide 11c ( $211 \mathrm{mg}, 1.0 \mathrm{mmol})$. Purification by flash column chromatography $\left(\mathrm{SiO}_{2}, 40 \%\right.$ acetone/pentane) afforded sulfoximine 12c (131 mg, $54 \%)$ as a white solid; $\mathrm{R}_{f}=0.24$ (40\% acetone/pentane); $\mathrm{mp}=125-127^{\circ} \mathrm{C}$; IR (film) $/ \mathrm{cm}^{-1} 3283,1468,1315,1241,1212,1051$, 980, 764; ${ }^{1} \mathrm{H}$ NMR $\left(400 \mathrm{MHz}, \mathrm{CDCl}_{3}\right) \delta 8.27-8.18(\mathrm{~m}, 1 \mathrm{H}), 8.07-7.98(\mathrm{~m}, 1 \mathrm{H}), 7.69-7.56(\mathrm{~m}, 2 \mathrm{H}), 4.23-4.09(\mathrm{~m}, 3 \mathrm{H}), 3.79-3.68(\mathrm{~m}, 1 \mathrm{H}), 3.66-$ $3.60(\mathrm{~m}, 2 \mathrm{H}) ;{ }^{13} \mathrm{C}\left\{{ }^{1} \mathrm{H}\right\} \mathrm{NMR}\left(101 \mathrm{~Hz}, \mathrm{CDCl}_{3}\right) \delta 169.2,152.7,137.5,127.9,127.7,125.4,122.3,57.8,56.6$; HRMS (APCI+) m/z: Calcd for $\mathrm{C}_{9} \mathrm{H}_{11} \mathrm{~N}_{2} \mathrm{O}_{2} \mathrm{~S}_{2}+[\mathrm{M}+\mathrm{H}]+:$ 243.0256; Found: 243.0260 .

Benzyl(2-hydroxyethyl)(imino)- $\lambda^{6}$-sulfanone (12d). The title compound was prepared according to General Procedure A employing sulfide $11 \mathrm{~d}(0.15 \mathrm{~mL}, 1.0 \mathrm{mmol})$. Purification by flash column chromatography $\left(\mathrm{SiO}_{2}, 1-5 \% \mathrm{MeOH} / \mathrm{CH}_{2} \mathrm{Cl}_{2}\right)$ afforded sulfoximine 12d $(180 \mathrm{mg}$, 91\%) as a white solid; $\mathrm{R}_{f}=0.10\left(4 \% \mathrm{MeOH} / \mathrm{CH}_{2} \mathrm{Cl}_{2}\right) ; \mathrm{mp}=94-96^{\circ} \mathrm{C}$; IR (film)/ $\mathrm{cm}^{-1} 3258,3202$ (broad), 2989, 2904, 2855, 1495. 1387, 1342, $1275,1234,1208,1167,1118,1081,1029,998,876,835,723,693 ;{ }^{1} \mathrm{H}$ NMR $\left(400 \mathrm{MHz}, \mathrm{DMSO}-\mathrm{d}_{6}\right) \delta 6.97-6.82(\mathrm{~m}, 5 \mathrm{H}), 4.61(\mathrm{t}, J=5.2 \mathrm{~Hz}$, $1 \mathrm{H}), 3.88(\mathrm{~s}, 2 \mathrm{H}), 3.16(\mathrm{~s}, 1 \mathrm{H}), 2.85(\mathrm{~s}, 2 \mathrm{H}), 2.01(\mathrm{q}, J=1.8 \mathrm{~Hz}, 2 \mathrm{H}) ;{ }^{13} \mathrm{C}$ NMR $(101 \mathrm{MHz}, \mathrm{DMSO}-\mathrm{d} 6) \delta 131.1(2 \times \mathrm{C}), 130.4,128.3(2 \times \mathrm{C}), 128.0$, 61.6, 55.6, 54.3; HRMS (APCI+) m/z: Calcd for $\mathrm{C}_{9} \mathrm{H}_{14} \mathrm{NO}_{2} \mathrm{~S}^{+}[\mathrm{M}+\mathrm{H}]^{+}: 200.0740$; Found: 200.0738 .

Imino(phenyl)(vinyl)- $\lambda^{6}$-sulfanone (13a) and $N$-(oxo(phenyl)(vinyl)- $\lambda^{6}$-sulfaneylidene)methanesulfonamide (14a). The title compounds were prepared according to General Procedure G employing sulfoximine 12a (141 mg, $0.76 \mathrm{mmol})$. Purification by flash column chromatography $\left(\mathrm{SiO}_{2}, 50 \%\right.$ EtOAc/pentane) afforded vinyl sulfoximine 13a (63 mg, 50\%) and mesylated vinyl sulfoximine 14a (11 mg, 6\%) both as colourless oils; Analytical data for vinyl sulfoximine 13a: $\mathrm{R}_{f}=0.23$ (50\% EtOAc/pentane); IR (film)/ $\mathrm{cm}^{-1} 3269,3064,1446,1223,1129,977$, 731, 693; ${ }^{1} \mathrm{H}$ NMR $\left(400 \mathrm{MHz}, \mathrm{CDCl}_{3}\right) \delta 8.04-7.94(\mathrm{~m}, 2 \mathrm{H}), 7.67-7.58(\mathrm{~m}, 1 \mathrm{H}), 7.57-7.51(\mathrm{~m}, 2 \mathrm{H}), 6.75(\mathrm{dd}, J=16.4,9.5 \mathrm{~Hz}, 1 \mathrm{H}), 6.43(\mathrm{dd}, J=$ 16.4, $0.6 \mathrm{~Hz}, 1 \mathrm{H}), 5.99$ (dd, $J=9.5,0.5 \mathrm{~Hz}, 1 \mathrm{H}), 2.88(\mathrm{~s}, 1 \mathrm{H}) ;{ }^{13} \mathrm{C}\left\{{ }^{1} \mathrm{H}\right\} \operatorname{NMR}\left(101 \mathrm{~Hz}, \mathrm{CDCl}_{3}\right) \delta 141.7,140.5,133.0,129.2(2 \times \mathrm{C}), 128.1(2 \times \mathrm{C})$, 126.6; Analytical data in agreement with those reported in the literature. ${ }^{30}$ Analytical data for mesylated vinyl sulfoximine 14a: $\mathrm{R}_{f}=0.29$ (50\% EtOAc/pentane); IR (film)/ $\mathrm{cm}^{-1} 3269,3060,1308,1230,1140,1099,1066,969,801,745 ;{ }^{1} \mathrm{H} \mathrm{NMR}\left(400 \mathrm{MHz}^{2} \mathrm{CDCl}_{3}\right) \delta 8.07-7.96(\mathrm{~m}$, 2H), 7.76-7.67 (m, 1H), 7.67-7.57 (m, 2H), $6.83(\mathrm{dd}, J=16.3,9.6 \mathrm{~Hz}, 1 \mathrm{H}), 6.52(\mathrm{dd}, J=16.3,1.3 \mathrm{~Hz}, 1 \mathrm{H}), 6.23(\mathrm{dd}, J=9.6,1.4 \mathrm{~Hz}, 1 \mathrm{H}), 3.18(\mathrm{~s}$, 
$3 \mathrm{H}) ;{ }^{13} \mathrm{C}\left\{{ }^{1} \mathrm{H}\right\} \mathrm{NMR}\left(101 \mathrm{~Hz}, \mathrm{CDCl}_{3}\right) \delta 137.5,137.0,134.5,129.7(2 \times \mathrm{C}), 129.4,128.0(2 \times \mathrm{C}), 45.4$; Analytical data in agreement with those reported in the literature.61

Imino(4-nitrophenyl)(vinyl)- $\lambda^{6}$-sulfanone (13b). The title compound was prepared according to General Procedure G employing sulfoximine $\mathbf{1 2 b}$ ( $57.5 \mathrm{mg}, 0.25 \mathrm{mmol})$. Purification by flash column chromatography $\left(\mathrm{SiO}_{2}, 0-10 \% \mathrm{Et}_{2} \mathrm{O} / \mathrm{CH}_{2} \mathrm{Cl}_{2}\right)$ afforded vinyl sulfoximine $\mathbf{1 3 b}$ $(26.5 \mathrm{mg}, 52 \%)$ as an off-white solid; $\mathrm{R}_{f}=0.35\left(10 \% \mathrm{Et}_{2} \mathrm{O} / \mathrm{CH}_{2} \mathrm{Cl}_{2}\right) ; \mathrm{mp}=95-97^{\circ} \mathrm{C}$; IR (film) $/ \mathrm{cm}^{-1} 3280,3105,3064,1607,1528,1349,1312$, $1230,1174,1141,977,857,738,705 ;{ }^{1} \mathrm{H}$ NMR $\left(400 \mathrm{MHz}, \mathrm{CDCl}_{3}\right) \delta 8.51-8.21(\mathrm{~m}, 2 \mathrm{H}), 8.25-8.09(\mathrm{~m}, 2 \mathrm{H}), 6.75(\mathrm{dd}, J=16.3,9.5 \mathrm{~Hz}, 1 \mathrm{H}), 6.53$ $(\mathrm{dd}, J=16.3,0.7 \mathrm{~Hz}, 1 \mathrm{H}), 6.12(\mathrm{dd}, J=9.4,0.7 \mathrm{~Hz}, 1 \mathrm{H}) ;{ }^{13} \mathrm{C}\left\{{ }^{1} \mathrm{H}\right\} \mathrm{NMR}\left(101 \mathrm{~Hz}, \mathrm{CDCl}_{3}\right) \delta 150.6,148.1,139.7,129.6(2 \times \mathrm{C}), 129.0,124.5(2 \times \mathrm{C})$; HRMS (ESI-TOF) m/z: Calcd for $\mathrm{C}_{8} \mathrm{H}_{9} \mathrm{~N}_{2} \mathrm{O}_{3} \mathrm{~S}$ [M+H]+: 213.0334; Found: 213.0339.

Benzo[d]thiazol-2-yl(imino)(vinyl)- $\lambda 6$-sulfanone (13c). The title compound was prepared according to General Procedure $G$ employing sulfoximine 12c (133 mg, $0.55 \mathrm{mmol})$. Purification by flash column chromatography $\left(\mathrm{SiO}_{2}, 30 \%\right.$ acetone/pentane) afforded vinyl sulfoximine 13c (48 mg, 39\%) as a white solid; $\mathrm{R}_{f}=0.39$ (30\% acetone/pentane); $\mathrm{mp}=99-101^{\circ} \mathrm{C}$; $\mathrm{IR}(\mathrm{film}) / \mathrm{cm}^{-1} 3261,1468,1315,1259,1237,1111$, 977, 760, 723; ${ }^{1} \mathrm{H}$ NMR (400 MHz, $\left.\mathrm{CDCl}_{3}\right) \delta 8.24-8.17(\mathrm{~m}, 1 \mathrm{H}), 8.02-7.95(\mathrm{~m}, 1 \mathrm{H}), 7.66-7.53(\mathrm{~m}, 2 \mathrm{H}), 7.02(\mathrm{dd}, J=16.4,9.5 \mathrm{~Hz}, 1 \mathrm{H}), 6.71(\mathrm{dd}$, $J=16.4,1.0 \mathrm{~Hz}, 1 \mathrm{H}), 6.28(\mathrm{dt}, J=9.5,0.9 \mathrm{~Hz}, 1 \mathrm{H}), 3.56(\mathrm{~s}, 1 \mathrm{H}) ;{ }^{13} \mathrm{C}\left\{{ }^{1} \mathrm{H}\right\} \mathrm{NMR}\left(101 \mathrm{~Hz}, \mathrm{CDCl}_{3}\right) \delta 169.5,153.0,137.7,137.5,131.0,127.7,127.4$, 125.3, 122.2; HRMS (APCI+) m/z: Calcd for $\mathrm{C}_{9} \mathrm{H}_{9} \mathrm{~N}_{2} \mathrm{OS}_{2}{ }^{+}[\mathrm{M}+\mathrm{H}]^{+}:$: 225.0151; Found: 225.0153.

Benzyl(imino)(vinyl)- $\lambda^{6}$-sulfanone (13d) and $N$-(benzyl(oxo)(vinyl)- $\lambda^{6}$-sulfaneylidene)methanesulfonamide (14d). The title compounds were prepared according to General Procedure G employing sulfoximine 12d (70 mg, $0.35 \mathrm{mmol})$. Purification by flash column chromatography (KP-Sil, $0 \%$ grading to $50 \%$ acetone in pentane) afforded vinyl sulfoximine $13 \mathbf{d}(22 \mathrm{mg}$, 35\%) as a colourless oil and mesylated vinyl sulfoximine 14d (26 mg, 29\%) as a white solid. Analytical data for vinyl sulfoximine 13d: $\mathrm{R}_{f}=0.30\left(50 \%\right.$ EtOAc/pentane); IR (film)/cm ${ }^{-1}$ $3273,1215,967,701 ;{ }^{1} \mathrm{H}$ NMR $\left(400 \mathrm{MHz}, \mathrm{DMSO}-\mathrm{d}_{6}\right) \delta$ 7.42-7.25 (m, 5H), $6.76(\mathrm{dd}, J=16.4,9.6 \mathrm{~Hz}, 1 \mathrm{H}), 6.07-5.92(\mathrm{~m}, 2 \mathrm{H}), 4.34(\mathrm{~s}, 2 \mathrm{H}), 3.93$ (br s, $1 \mathrm{H}) ;{ }^{13} \mathrm{C}\left\{{ }^{1} \mathrm{H}\right\}$ NMR $(101 \mathrm{MHz}$, DMSO-d $) \delta 138.9,131.0(2 \times \mathrm{C}), 130.0,128.1,128.0,127.7,61.4$; HRMS (ESI-TOF) m/z: Calcd for $\mathrm{C}_{9} \mathrm{H}_{12} \mathrm{NOS}^{+}[\mathrm{M}+\mathrm{H}]^{+}:$182.0640; Found: 182.0640. Analytical data for mesylated vinyl sulfoximine $14 \mathrm{~d}: \mathrm{R}_{f}=0.40\left(10 \% \mathrm{Et}_{2} \mathrm{O} / \mathrm{CH}_{2} \mathrm{Cl}_{2}\right) ; \mathrm{mp}=$ 139-140 ${ }^{\circ} \mathrm{C}$; IR (film)/cm ${ }^{-1} 3109,3070,2982,2933,1744,1532,1495,1457,1409,1383,1305,1230,1137,1066,969,897,783,746,701$; ${ }^{1} \mathrm{H}$ NMR $\left(400 \mathrm{MHz}, \mathrm{CDCl}_{3}\right) \delta 7.41-7.38(\mathrm{~m}, 5 \mathrm{H}), 6.53(\mathrm{dd}, J=16.3,9.7 \mathrm{~Hz}), 6.36-6.24(\mathrm{~m}, 2 \mathrm{H}), 4.73(\mathrm{~s}, 2 \mathrm{H}), 3.14(\mathrm{~s}, 3 \mathrm{H}) ;{ }^{13} \mathrm{C}\left\{{ }^{1} \mathrm{H}\right\} \mathrm{NMR}(101$ $\left.\left.\mathrm{MHz}, \mathrm{CDCl}_{3}\right) \delta 133.4,133.2,131.7(2 \times \mathrm{C}), 129.9,129.2(2 \times \mathrm{C}), 126.3,62.3,45.4 ; \mathrm{HRMS}_{(\mathrm{APCI}}{ }^{+}\right) \mathrm{m} / \mathrm{z}$ : Calcd for $\mathrm{C}_{10} \mathrm{H}_{14} \mathrm{NO}_{3} \mathrm{~S}_{2}+[\mathrm{M}+\mathrm{H}]^{+}: 260.0415$; Found: 260.0408.

Terminal vinyl sulfonimidamides 18a-18d.

1,2-Bis(2-((tetrahydro-2H-pyran-2-yl)oxy)ethyl)disulfane (16). Pyridinium $p$-toluenesulfonate (2.00 g, $7.90 \mathrm{mmol})$ was added to a solution of 2-hydroxyethyl disulfide $(10.0 \mathrm{~mL}, 81.7 \mathrm{mmol})$ and 3,4-dihydropyran $(30.0 \mathrm{~mL}, 328 \mathrm{mmol})$ in $\mathrm{CH}_{2} \mathrm{Cl}_{2}(100 \mathrm{~mL})$ at $0{ }^{\circ} \mathrm{C}$. The solution was allowed to warm to room temperature and was stirred for $18 \mathrm{~h}$. The solvent was removed in vacuo and the crude residue was purified by flash column chromatography (KP-Sil, $0 \%$ grading to $50 \% \mathrm{Et}_{2} \mathrm{O}$ in pentane, eluted at $10 \%$ ) which afforded disulfide $\mathbf{1 6}$ (22.7 g, $86 \%$ ) as a colourless oil; $\mathrm{R}_{f}=0.44\left(25 \% \mathrm{Et}_{2} \mathrm{O}\right.$ in pentane); IR (film)/ $\mathrm{cm}^{-1} 3008,2941,2870,1438,1349,1200,1118,1077,1025,753 ;{ }^{1} \mathrm{H} \mathrm{NMR}(400$ $\left.\mathrm{MHz} \mathrm{CDCl}_{3}\right) \delta 4.64(\mathrm{dd}, J=4.2,2.9 \mathrm{~Hz}, 2 \mathrm{H}), 4.04-3.92(\mathrm{~m}, 2 \mathrm{H}), 3.88(\mathrm{ddd}, J=11.2,8.0,3.5 \mathrm{~Hz}, 2 \mathrm{H}), 3.69(\mathrm{dt}, J=10.4,6.7 \mathrm{~Hz}, 2 \mathrm{H}), 3.58-3.47$ $(\mathrm{m}, 2 \mathrm{H}), 3.01-2.85(\mathrm{~m}, 4 \mathrm{H}), 1.89-1.66(\mathrm{~m}, 4 \mathrm{H}), 1.65-1.46(\mathrm{~m}, 8 \mathrm{H}) ;{ }^{13} \mathrm{C}\left\{{ }^{1} \mathrm{H}\right\} \mathrm{NMR}(101 \mathrm{~Hz}, \mathrm{CDCl} 3) \delta 99.1(2 \times \mathrm{C}), 66.1(2 \times \mathrm{C}), 62.4(2 \times \mathrm{C}), 39.2$ $(2 \times \mathrm{C}), 30.7(2 \times \mathrm{C}), 25.6(2 \times \mathrm{C}), 19.5(2 \times \mathrm{C})$; HRMS $\left(\mathrm{APCI}^{+}\right) \mathrm{m} / \mathrm{z}$ Calcd for $\mathrm{C}_{14} \mathrm{H}_{26} \mathrm{O}_{4} \mathrm{~S}_{2}{ }^{+}[\mathrm{M}]^{+}, 322.1267$; found, 322.1276 .

4-Phenyl-1-(2-((tetrahydro-2H-pyran-2-yl)oxy)ethylsulfonimidoyl)piperidine (17a). The title compound was prepared according to General Procedure H employing silver nitrate (603 mg, $4.0 \mathrm{mmol}$ ), triethylamine (554 $\mu \mathrm{L}, 4.0 \mathrm{mmol}$ ), disulfide 16 (644 mg, $2.0 \mathrm{mmol}$ ) and 4-phenyl piperidine (427 mg, $2.66 \mathrm{mmol}$ ) in $\mathrm{MeOH}(26 \mathrm{~mL}$ ) followed by ammonium carbamate (223 mg, $2.86 \mathrm{mmol})$, iodosylbenzene (788 $\mathrm{mg}, 3.58 \mathrm{mmol})$ and acetic acid $(80 \mu \mathrm{L}, 1.4 \mathrm{mmol})$ in $i \operatorname{PrOH}(7 \mathrm{~mL})$. Purification by flash column chromatography (KP-Sil, $50 \%$ grading to $100 \%$ EtOAc in pentane, product eluted at $100 \%$ ) afforded sulfonimidamide $17 \mathrm{a}(400 \mathrm{mg}, 57 \%$ over 2 steps, dr $1: 1)$ as a colourless oil; $\mathrm{R}_{f}=$ 0.40 (100\% EtOAc); IR (film)/ $\mathrm{cm}^{-1} 3280,2937,2847,1244,1118,1028,931 ;{ }^{1} \mathrm{H}$ NMR $\left(400 \mathrm{MHz}, \mathrm{CDCl}_{3}\right) \delta 7.36-7.27$ (m, 2H), 7.25-7.14 (m, $3 \mathrm{H}), 4.71-4.57(\mathrm{~m}, 1 \mathrm{H}), 4.24-4.09(\mathrm{~m}, 1 \mathrm{H}), 4.09-3.96(\mathrm{~m}, 2 \mathrm{H}), 3.96-3.81(\mathrm{~m}, 2 \mathrm{H}), 3.60-3.48(\mathrm{~m}, 1 \mathrm{H}), 3.40-3.16(\mathrm{~m}, 2 \mathrm{H}), 2.93-2.75(\mathrm{~m}, 2 \mathrm{H})$, $2.57(\mathrm{td}, J=12.1,3.0 \mathrm{~Hz}, 1 \mathrm{H}), 2.28(\mathrm{~d}, J=7.8 \mathrm{~Hz}, 1 \mathrm{H}), 2.03-1.88(\mathrm{~m}, 2 \mathrm{H}), 1.88-1.64(\mathrm{~m}, 5 \mathrm{H}), 1.64-1.43(\mathrm{~m}, 4 \mathrm{H}) ;{ }^{13} \mathrm{C}\left\{{ }^{1} \mathrm{H}\right\} \mathrm{NMR}(101 \mathrm{~Hz}, \mathrm{CDCl} 3)$ $\delta 145.3,128.8,126.9,126.7,99.6,99.5,62.8,62.0,61.8,50.1,50.0,47.5,47.5,47.2,47.1,42.5,33.8,33.6,33.5,30.8,30.7,25.4,19.8$; HRMS (ESI-TOF) $m / z$ Calcd for $\mathrm{C}_{18} \mathrm{H}_{29} \mathrm{~N}_{2} \mathrm{O}_{3} \mathrm{~S}^{+}[\mathrm{M}+\mathrm{H}]^{+}, 353.1899$; found 353.1901 .

4-Methyl-2-phenyl-1-(2-((tetrahydro-2H-pyran-2-yl)oxy)ethylsulfonimidoyl)piperazine (17b). The title compound was prepared according to General Procedure H employing 1-methyl-3-phenylpiperazine (587 mg, $3.3 \mathrm{mmol})$, triethylamine (693 $\mu \mathrm{L}, 5.0 \mathrm{mmol})$, silver nitrate $(849 \mathrm{mg}, 5.0 \mathrm{mmol}$ and disulfide $16(806 \mathrm{mg}, 2.5 \mathrm{mmol})$ in $\mathrm{MeOH}(25 \mathrm{~mL})$ followed by ammonium carbamate (390 mg, $5.0 \mathrm{mmol})$, iodosylbenzene $(1.37 \mathrm{~g}, 6.25 \mathrm{mmol})$ and acetic acid $(143 \mu \mathrm{L}, 2.5 \mathrm{mmol})$ in $i \mathrm{PrOH}(13 \mathrm{~mL})$. Purification by flash column chromatography (SiO 2 , 70\% EtOAc in pentane) afforded sulfonimidamide $\mathbf{1 7} \mathbf{b}$ (292 $\mathrm{mg}, 32 \%$ over 2 steps) as a complex mixture of diastereomers as a brown oil; $\mathrm{R}_{f}=0.11$ (70\% EtOAc in pentane); IR (film)/ $\mathrm{cm}^{-1} 3272,2937,2870,2791,1453,1244,1148,1118,1029,976,917 ;{ }^{1} \mathrm{H} \mathrm{NMR}(400 \mathrm{MHz}, \mathrm{CDCl} 3)$ $\delta 7.71-7.64(\mathrm{~m}, 4 \mathrm{H}), 7.38-7.32(\mathrm{~m}, 4 \mathrm{H}), 7.31-7.27(\mathrm{~m}, 2 \mathrm{H}), 5.14(\mathrm{~d}, J=3.4 \mathrm{~Hz}, 1 \mathrm{H}), 5.10(\mathrm{~d}, J=4.3 \mathrm{~Hz}, 1 \mathrm{H}), 4.60-4.53(\mathrm{~m}, 2 \mathrm{H}), 4.13-4.01(\mathrm{~m}$, $2 \mathrm{H}), 3.89-3.74(\mathrm{~m}, 6 \mathrm{H}), 3.54-3.45(\mathrm{~m}, 2 \mathrm{H}), 3.41-3.05(\mathrm{~m}, 6 \mathrm{H}), 2.98-2.78(\mathrm{~m}, 4 \mathrm{H}), 2.52-2.47(\mathrm{~m}, 2 \mathrm{H}), 2.31-2.28(\mathrm{~m}, 6 \mathrm{H}), 2.30(\mathrm{~s}, 2 \mathrm{H}), 2.22-$ $2.11(\mathrm{~m}, 2 \mathrm{H}), 1.84-1.64(\mathrm{~m}, 4 \mathrm{H}), 1.64-1.45(\mathrm{~m}, 8 \mathrm{H}) ;{ }^{13} \mathrm{C}\left\{{ }^{1} \mathrm{H}\right\} \mathrm{NMR}\left(101 \mathrm{~Hz}, \mathrm{CDCl}_{3}\right) \delta 139.7,128.5,128.4,128.3,128.13,128.10,127.5,127.4$, $99.1,99.0,62.3,62.2,61.93,61.89,59.1,58.5,56.2,55.9,55.4,55.2,53.64,53.63,46.30,46.27,42.1,41.9,30.4,30.3,25.2,19.32,19.29 ;$ HRMS (ESI-TOF) m/z Calcd for $\mathrm{C}_{18} \mathrm{H}_{30} \mathrm{~N}_{3} \mathrm{O}_{3} \mathrm{~S}$ [M + H]+, 368.2008; found; 368.2006.

$N$-(3-(10,11-Dihydro-5H-dibenzo[a,d] [7] annulen-5-ylidene)propyl)- $N$-methyl-2-((tetrahydro-2H-pyran-2-yl)oxy)ethane-1-sulfonimidamide (17c). The title compound was prepared according to General Procedure H employing nortriptyline hydrochloride (1.00 g, 3.33 mmol), triethylamine $(1.20 \mathrm{~mL}, 8.75 \mathrm{mmol})$, silver nitrate (849 mg, $5.0 \mathrm{mmol})$ and disulfide 16 (806 mg, $2.5 \mathrm{mmol}) \mathrm{in} \mathrm{MeOH} \mathrm{(25} \mathrm{mL)} \mathrm{fol-}$ lowed by ammonium carbamate $(390 \mathrm{mg}, 5.0 \mathrm{mmol})$, iodosylbenzene $(1.37 \mathrm{~g}, 6.25 \mathrm{mmol})$ and acetic acid $(143 \mu \mathrm{L}, 2.5 \mathrm{mmol}) \mathrm{in} i \operatorname{PrOH}(13$ $\mathrm{mL})$. Purification by flash column chromatography $\left(\mathrm{SiO}_{2}, 20 \%\right.$ acetone in pentane) afforded sulfonimidamide $17 \mathrm{c}$ ( $375 \mathrm{mg}, 33 \%$ over $2 \mathrm{steps}$, $\mathrm{dr} 1: 1)$ as a brown oil; $\mathrm{R}_{f}=0.18$ (20\% acetone in pentane); IR (film)/ $\mathrm{cm}^{-1} 3309,3015,2937,1643,1483,1442,1244,1121,1077,1028,969$, 766; ${ }^{1} \mathrm{H}$ NMR (400 MHz, CDCl 3 ) $\delta 7.29-7.25(\mathrm{~m}, 1 \mathrm{H}), 7.23-7.13(\mathrm{~m}, 6 \mathrm{H}), 7.06-7.03(\mathrm{~m}, 1 \mathrm{H}), 5.85(\mathrm{td}, J=7.4,1.4 \mathrm{~Hz}, 1 \mathrm{H}), 4.60(\mathrm{dd}, J=4.5,3.0$ $\mathrm{Hz}, 1 \mathrm{H}), 4.10(\mathrm{ddt}, J=10.9,9.3,6.1 \mathrm{~Hz}, 1 \mathrm{H}), 3.90-3.80(\mathrm{~m}, 2 \mathrm{H}), 3.65-3.13(\mathrm{~m}, 9 \mathrm{H}), 2.97(\mathrm{~s}, 1 \mathrm{H}), 2.76(\mathrm{~s}, 3 \mathrm{H}), 2.44-2.34(\mathrm{~m}, 2 \mathrm{H}), 1.83-1.70(\mathrm{~m}$, 2H), 1.61-1.49 (m, 4H); ${ }^{13} \mathrm{C}\left\{{ }^{1} \mathrm{H}\right\} \mathrm{NMR}\left(101 \mathrm{~Hz}, \mathrm{CDCl}_{3}\right) \delta 145.1,140.8,139.7,139.4,137.0,130.1,128.5,128.1,128.0,127.6,127.3,127.2$, 126.1, 125.7, 99.2, 62.6, 61.8, 61.6, 50.5, 50.3, 33.7, 32.0, 30.5, 28.6, 25.2, 19.5; HRMS (ESI-TOF) m/z Calcd for $\mathrm{C}_{26} \mathrm{H}_{35} \mathrm{~N}_{2} \mathrm{O}_{3} \mathrm{~S}\left[\mathrm{M}+\mathrm{H}^{+}, 455.2368\right.$; found, 455.2354 .

$\mathrm{N}$-Benzyl- $\mathrm{N}$-methyl-2-((tetrahydro-2H-pyran-2-yl)oxy)ethane-1-sulfonimidamide (17d). The title compound was prepared according to General Procedure H employing silver nitrate $(603 \mathrm{mg}, 4.0 \mathrm{mmol})$, triethylamine (554 $\mu \mathrm{L}, 4.0 \mathrm{mmol}), N$-methylbenzylamine $(346 \mu \mathrm{L}, 2.66$ 
mmol) and disulfide 16 (645.0 mg, $2.0 \mathrm{mmol})$ in $\mathrm{MeOH}(20 \mathrm{~mL}$ ) followed by ammonium carbamate (390 mg, $5.0 \mathrm{mmol})$, iodosylbenzene $(1.37 \mathrm{~g}, 6.25 \mathrm{mmol})$ and acetic acid $(143 \mu \mathrm{L}, 2.5 \mathrm{mmol})$ in $i \operatorname{PrOH}(13 \mathrm{~mL})$. Purification by flash column chromatography (KP-Sil $0 \%$ grading to $100 \% \mathrm{MeOH}$ in $\mathrm{CH}_{2} \mathrm{Cl}_{2}$, product eluted at 7\%) afforded sulfonimidamide $\mathbf{1 7 d}(324 \mathrm{mg}, 52 \%$ over 2 steps, dr 1:1) as a yellow oil; $\mathrm{R} f=0.28$ (100\% EtOAc); IR (film)/cm-1 3280, 2937, 2870, 1454, 1349, 1245, 1122, 1077, 1028, 976, 731; ${ }^{1} \mathrm{H} \mathrm{NMR}\left(400 \mathrm{MHz}^{-} \mathrm{CDCl}_{3}\right) \delta 7.30-7.27$ (m, $5 \mathrm{H}), 4.65(\mathrm{~m}, 1 \mathrm{H}), 4.52-4.32(\mathrm{~m}, 2 \mathrm{H}), 4.24-4.10(\mathrm{~m}, 1 \mathrm{H}), 3.97-3.84(\mathrm{~m}, 2 \mathrm{H}), 3.58-3.50(\mathrm{~m}, 1 \mathrm{H}), 3.47-3.25(\mathrm{~m}, 2 \mathrm{H}), 2.81(\mathrm{~d}, J=3.2 \mathrm{~Hz}, 3 \mathrm{H})$, $2.34(\mathrm{~d}, J=8.3 \mathrm{~Hz}, 1 \mathrm{H}), 1.92-1.71(\mathrm{~m}, 2 \mathrm{H}) 1.66-1.47(\mathrm{~m}, 4 \mathrm{H}) ;{ }^{13} \mathrm{C}\left\{{ }^{1} \mathrm{H}\right\} \mathrm{NMR}\left(101 \mathrm{~Hz}, \mathrm{CDCl}_{3}\right) \delta 137.0,128.8,128.1,127.8,99.5,62.8,62.0,61.8$, 54.9, 50.9, 35.4, 30.8, 25.4, 19.8; HRMS (ESI-TOF) $m / z$ Calcd for $\mathrm{C}_{15} \mathrm{H}_{25} \mathrm{~N}_{2} \mathrm{O}_{3} \mathrm{~S}^{+}[\mathrm{M}+\mathrm{H}]^{+}, 313.1580$; found 313.1578 .

4-Phenyl-1-(vinylsulfonimidoyl)piperidine (18a). The title compound was prepared according to General Procedure I employing sulfonimidamide $17 \mathrm{a}(32 \mathrm{mg}, 0.09 \mathrm{mmol}$ ). Purification by flash column chromatography (KP-Sil, $50 \%$ grading to $100 \%$ EtOAc in pentane, product eluted at $100 \%$ ) to give sulfonimidamide $18 a\left(17 \mathrm{mg}, 70 \%\right.$ over 2 steps) as a colourless oil; $\mathrm{R}_{f}=0.50(100 \% \mathrm{EtOAc})$; IR (film) $/ \mathrm{cm}^{-1} 3287$, 2937, 1244, 1051, 924, 734; ${ }^{1} \mathrm{H}$ NMR $\left(400 \mathrm{MHz}, \mathrm{CDCl}_{3}\right) \delta 7.37-7.27(\mathrm{~m}, 2 \mathrm{H}), 7.25-7.13(\mathrm{~m}, 3 \mathrm{H}), 6.52(\mathrm{dd}, J=16.6,9.9 \mathrm{~Hz}, 1 \mathrm{H}), 6.26(\mathrm{~d}, J=16.6$ $\mathrm{Hz}, 1 \mathrm{H}), 6.03(\mathrm{~d}, J=9.9 \mathrm{~Hz}, 1 \mathrm{H}), 4.02(\mathrm{ddt}, J=11.9,4.4,2.5 \mathrm{~Hz}, 1 \mathrm{H}), 3.92(\mathrm{ddt}, J=11.9,4.6,2.4 \mathrm{~Hz}, 1 \mathrm{H}), 2.72-2.44(\mathrm{~m}, 3 \mathrm{H}), 2.37(\mathrm{~s}, 1 \mathrm{H}), 2.01-$ $1.87(\mathrm{~m}, 2 \mathrm{H}), 1.87-1.72(\mathrm{~m}, 2 \mathrm{H}) ;{ }^{13} \mathrm{C}\left\{{ }^{1} \mathrm{H}\right\}$ NMR $\left(101 \mathrm{~Hz}, \mathrm{CDCl}_{3}\right) \delta 145.2,133.4,128.7(2 \times \mathrm{C}), 127.7,126.9(2 \times \mathrm{C}), 126.7,47.8,47.4,42.3,33.4$, 33.1; HRMS (ESI-TOF) $\mathrm{m} / z$ Calcd for $\mathrm{C}_{13} \mathrm{H}_{19} \mathrm{~N}_{2} \mathrm{OS}^{+}[\mathrm{M}+\mathrm{H}]^{+}, 251.1218$; found 251.1207.

4-Methyl-2-phenyl-1-(vinylsulfonimidoyl)piperazine (18b). The title compound was prepared according to General Procedure I employing sulfonimidamide $\mathbf{1 7 b}$ ( $238 \mathrm{mg}, 0.65 \mathrm{mmol})$. Purification by flash column chromatography $\left(\mathrm{SiO}_{2}, 20 \%\right.$ acetone in pentane) afforded sulfonimidamide $18 \mathrm{~b}$ (70 mg, 42\% over 2 steps, $\mathrm{dr} 1: 2)$ as a brown oil; $\mathrm{R}_{f}=0.27$ (20\% acetone in pentane); IR (film)/ $\mathrm{cm}^{-1} 3272,2937,2795$, $1453,1267,1244,1148,973,924 ;{ }^{1} \mathrm{H}$ NMR $\left(400 \mathrm{MHz}, \mathrm{CDCl}_{3}\right) \delta 7.69$ (dd, $\left.J=7.3,1.9 \mathrm{~Hz}, 2 \mathrm{H}\right), 7.63(\mathrm{dd}, J=7.4,1.8 \mathrm{~Hz}, 2 \mathrm{H}), 7.36-7.32(\mathrm{~m}, 4 \mathrm{H})$, 7.31-7.25 (m, 2H), 6.27-6.09 (m, 4H), 5.76-5.71 (m, 2H), 5.05-5.02 (m, 2H), 3.72-3.67 (m, 2H), 3.35-3.13 (m, 4H), 2.84-2.77 (m, 2H), 2.51$2.47(\mathrm{~m}, 2 \mathrm{H}), 2.33(\mathrm{~s}, 2 \mathrm{H}), 2.30-2.17(\mathrm{~m}, 6 \mathrm{H}), 2.24-2.13(\mathrm{~m}, 2 \mathrm{H}) ;{ }^{13} \mathrm{C}\left\{{ }^{1} \mathrm{H}\right\} \mathrm{NMR}\left(101 \mathrm{~Hz}, \mathrm{CDCl}_{3}\right) \delta 139.6,137.1,136.4,128.6,128.4,128.3$, 128.2, 127.5, 125.3, 125.0, 58.8, 57.9, 56.3, 56.2, 54.9, 54.8, 46.3, 42.1, 41.9; HRMS (ESI-TOF) m/z Calcd for $\mathrm{C}_{13} \mathrm{H}_{20} \mathrm{~N}_{3} \mathrm{OS}$ [M + H]+, 266.1327; found, 266.1334 .

$N$-(3-(10,11-Dihydro-5H-dibenzo[a,d][7]annulen-5-ylidene)propyl)- $N$-methylethenesulfonimidamide (18c). The title compound was prepared according to General Procedure I employing sulfonimidamide 17c (516 mg, $1.13 \mathrm{mmol}$ ). Purification by flash column chromatography $\left(\mathrm{SiO}_{2}, 50 \%\right.$ EtOAc in pentane) afforded sulfonimidamide 18c (188 mg, 47\%) as a brown oil; $\mathrm{R}_{f}=0.33$ (50\% EtOAc in pentane); IR (film) $/ \mathrm{cm}^{-1} 3309,3060,3015,2914,1483,1453,1267,1107,965,742 ;{ }^{1} \mathrm{H}$ NMR $\left(400 \mathrm{MHz}, \mathrm{CDCl}_{3}\right) \delta$ 7.29-7.27 (m, 1H), 7.26-7.09 (m, 6H), $7.08-7.02(\mathrm{~m}, 1 \mathrm{H}), 6.42(\mathrm{dd}, J=16.6,9.8 \mathrm{~Hz}, 1 \mathrm{H}), 6.19(\mathrm{~d}, J=16.6 \mathrm{~Hz}, 1 \mathrm{H}), 5.90(\mathrm{~d}, J=9.9 \mathrm{~Hz}, 1 \mathrm{H}), 5.85(\mathrm{t}, J=7.5 \mathrm{~Hz}, 1 \mathrm{H}), 3.46-3.23(\mathrm{~m}, 3 \mathrm{H})$, 3.17-3.05 (m, 1H), 3.02-2.92 (m, 1H), 2.85-2.75 (m, 1H), 2.71-2.60 (m, 3H), 2.44-2.32 (m, 2H), $2.09(\mathrm{~s}, 1 \mathrm{H}) ;{ }^{13} \mathrm{C}\left\{{ }^{1} \mathrm{H}\right\} \mathrm{NMR}^{2}(101 \mathrm{~Hz}, \mathrm{CDCl} 3) \delta$ 145.2, 140.7, 139.7, 139.4, 137.0, 133.4, 130.1, 128.5, 128.1, 128.0, 127.6, 127.23, 127.20, 126.6, 126.1, 125.8, 50.5 (2× C), 33.7, 31.9, 28.3; HRMS (ESI-TOF) m/z Calcd for $\mathrm{C}_{21} \mathrm{H}_{25} \mathrm{~N}_{2} \mathrm{OS}[\mathrm{M}+\mathrm{H}]^{+}$, 353.1688; found, 353.1682 .

$N$-Benzyl- $N$-methylethenesulfonimidamide (18d). The title compound was prepared according to General Procedure I employing sulfonimidamide $\mathbf{1 7 d}(144 \mathrm{mg}, 0.46 \mathrm{mmol})$. Purification by flash column chromatography (KP-Sil, 30\% grading to 100\% EtOAc/pentane, eluted at $50 \%)$ gave sulfonimidamide $\mathbf{1 8 d}\left(22 \mathrm{mg}, 23 \%\right.$ over 2 steps) as a pale yellow oil; $\mathrm{R}_{f}=0.25(100 \%$ EtOAc); IR (film)/cm-1 $3280,3030,1358$, $1243,1102,969,733 ;{ }^{1} \mathrm{H}$ NMR $\left(400 \mathrm{MHz}, \mathrm{CDCl}_{3}\right) \delta 7.43-7.27(\mathrm{~m}, 5 \mathrm{H}), 6.56(\mathrm{dd}, J=16.6,9.9 \mathrm{~Hz}, 1 \mathrm{H}), 6.31(\mathrm{~d}, J=16.6 \mathrm{~Hz}, 1 \mathrm{H}), 6.03(\mathrm{~d}, J=9.9$ $\mathrm{Hz}, 1 \mathrm{H}), 4.30(\mathrm{~m}, 2 \mathrm{H}), 2.73(\mathrm{~s}, 3 \mathrm{H}), 2.47(\mathrm{~s}, 1 \mathrm{H}) ;{ }^{13} \mathrm{C}\left\{{ }^{1} \mathrm{H}\right\} \mathrm{NMR}\left(101 \mathrm{~Hz}, \mathrm{CDCl}_{3}\right) \delta 136.6,133.5,128.8(2 \times \mathrm{C}), 128.3(2 \times \mathrm{C}), 127.9,127.2,55.0$, 35.3; HRMS (ESI-TOF) $\mathrm{m} / \mathrm{z}$ Calcd for $\mathrm{C}_{21} \mathrm{H}_{25} \mathrm{~N}_{2} \mathrm{OS}$ [M + H] $]^{+}, 211.0900$; found, 211.0896.

$N$-Propargyl vinyl sulfoximines and sulfonimidamides 19a-19g. Phenyl(prop-2-yn-1-ylimino)(vinyl)- $\lambda^{6}$-sulfanone (19a). The title compound was prepared according to General Procedure J employing sulfoximine 11a (36 mg, $0.21 \mathrm{mmol}$ ). Purification by flash column chromatography (KP-Sil, $0 \%$ grading to $50 \%$ EtOAc/pentane, product eluted at $25 \%$ ) afforded sulfoximine 19a (25 mg, $57 \%$ ) as a colourless oil; $\mathrm{R}_{f}=0.45\left(50 \%\right.$ EtOAc in pentane); IR (film) $\left./ \mathrm{cm}^{-1} 3295,2321,1446,1267,1216,1131,971,742 ;{ }^{1} \mathrm{H} \mathrm{NMR}(400 \mathrm{MHz}, \mathrm{CDCl})_{3}\right) \delta 7.95-7.90(\mathrm{~m}$, $2 \mathrm{H}), 7.62-7.51(\mathrm{~m}, 3 \mathrm{H}), 6.68(\mathrm{dd}, J=16.5,9.6 \mathrm{~Hz}, 1 \mathrm{H}), 6.38(\mathrm{~d}, J=16.5 \mathrm{~Hz}, 1 \mathrm{H}), 6.03(\mathrm{~d}, J=9.9 \mathrm{~Hz}, 1 \mathrm{H}), 3.87(\mathrm{dd}, J=17.2,2.5 \mathrm{~Hz}, 1 \mathrm{H}), 3.79(\mathrm{dd}$, $J=17.3,2.5 \mathrm{~Hz}, 1 \mathrm{H}), 2.19(\mathrm{t}, J=2.5 \mathrm{~Hz}, 1 \mathrm{H}) ;{ }^{13} \mathrm{C}\left\{{ }^{1} \mathrm{H}\right\} \mathrm{NMR}\left(101 \mathrm{~Hz}, \mathrm{CDCl}_{3}\right) \delta 138.5,138.4,133.3,129.5(2 \times \mathrm{C}), 129.0(2 \times \mathrm{C}), 128.2,83.0,70.6$ 32.6; HRMS (ESI-TOF) $m / z$ Calcd for $\mathrm{C}_{11} \mathrm{H}_{12} \mathrm{NOS}^{+}[\mathrm{M}+\mathrm{H}]^{+}, 206.0640$; found, 206.0647.

(E)-Phenyl(prop-2-yn-1-ylimino)(styryl)- $\lambda^{6}$-sulfanone (19b). The title compound was prepared according to General Procedure J employing sulfoximine $5 \mathrm{a}$ ( $36 \mathrm{mg}, 0.15 \mathrm{mmol}$ ). Purification by flash chromatography (KP-Sil, $0 \%$ grading to $100 \%$ EtOAc/Hexane, eluted at $35 \%$ EtOAc) afforded 19b (23 mg, 55\%) as a dark orange oil; $\mathrm{R}_{f}=0.64$ (50\% EtOAc/Pentane); IR (film)/ $\mathrm{cm}^{-1}$ 3295, 3060, 1610, 1446, 1267, 1215, $1133,1081,745,686 ;{ }^{1} \mathrm{H}$ NMR $\left(400 \mathrm{MHz}, \mathrm{CDCl}_{3}\right) \delta 8.01-7.98(\mathrm{~m}, 2 \mathrm{H}), 7.62-7.52(\mathrm{~m}, 4 \mathrm{H}), 7.48-7.46(\mathrm{~m}, 2 \mathrm{H}), 7.41-7.36(\mathrm{~m}, 3 \mathrm{H}), 6.89(\mathrm{~d}, J=$ $15.3 \mathrm{~Hz}, 1 \mathrm{H}), 3.91(\mathrm{dd}, J=14.8,2.5 \mathrm{~Hz}, 1 \mathrm{H}) 3.81(\mathrm{dd}, J=14.8,2.6 \mathrm{~Hz}, 1 \mathrm{H}), 2.23(\mathrm{t}, J=2.53 \mathrm{~Hz}, 1 \mathrm{H}) ;{ }^{13} \mathrm{C}\left\{{ }^{1} \mathrm{H}\right\} \mathrm{NMR}(101 \mathrm{~Hz}, \mathrm{CDCl} 3) \delta 142.9,139.3$, 133.0, 132.7, 131.0, $129.4(2 \times \mathrm{C}), 129.0(2 \times \mathrm{C}), 128.6(2 \times \mathrm{C}), 128.5(2 \times \mathrm{C}), 127.2,83.2,70.5,32.7 ; \mathrm{HRMS}(\mathrm{ESI}-\mathrm{TOF}) \mathrm{m} / z \mathrm{Calcd}$ for $\mathrm{C}_{17} \mathrm{H}_{16} \mathrm{NOS}^{+}$ $[\mathrm{M}+\mathrm{H}]^{+}$, 282.0953; found, 282.0960.

(E)-(2-Cyclohexylvinyl)(phenyl)(prop-2-yn-1-ylimino)- $\lambda 6$-sulfanone (19c).The title compound was prepared according to General Procedure J employing sulfoximine $\mathbf{5 b}(40 \mathrm{mg}, 0.16 \mathrm{mmol})$. Purification via flash column chromatography (KP-Sil, $0 \%$ grading to $100 \%$ EtOAc/Petroleum ether, eluted at 35\% EtOAc) afforded 19c (35 mg, 77\%) as a clear oil; $\mathrm{R}_{f}=0.61\left(50 \%\right.$ EtOAc/Pentane); IR (film)/cm ${ }^{-1} 3295$, 2926, 2851, 1446, 1267, 1218, 1133, 969, 752, 689; ${ }^{1} \mathrm{H}$ NMR $\left(400 \mathrm{MHz}, \mathrm{CDCl}_{3}\right) \delta$ 7.91-7.89 (m, 2H), 7.60-7.56 (m, 1H), 7.54-7.50 (m, 2H), $6.83(\mathrm{dd}, J=8.7,6.3 \mathrm{~Hz}, 1 \mathrm{H}), 6.28(\mathrm{~d}, J=15.2 \mathrm{~Hz}, 1 \mathrm{H}), 3.81(\mathrm{dd}, J=14.7,2.6 \mathrm{~Hz}, 1 \mathrm{H}), 3.71(\mathrm{dd}, J=14.9,2.5 \mathrm{~Hz}, 1 \mathrm{H}), 2.20(\mathrm{t}, J=2.5 \mathrm{~Hz}, 1 \mathrm{H})$, 2.18-2.12 (m, 1H) $1.74(\mathrm{~m}, 4 \mathrm{H}) 1.67-1.63(\mathrm{~m}, 1 \mathrm{H}), 1.30-1.09(\mathrm{~m}, 5 \mathrm{H}) ;{ }^{13} \mathrm{C}\left\{{ }^{1} \mathrm{H}\right\} \mathrm{NMR}\left(101 \mathrm{~Hz}, \mathrm{CDCl}_{3}\right) \delta 152.1,139.3,132.8,129.3(2 \times \mathrm{C}), 128.6$ $(2 \times \mathrm{C}), 127.8,83.2,70.3,40.0,32.6,31.4,31.2,25.7,25.6(2 \times \mathrm{C})$; HRMS (ESI-TOF) $\mathrm{m} / z$ Calcd for $\mathrm{C}_{17} \mathrm{H}_{22} \mathrm{NOS}^{+}\left[\mathrm{M}+\mathrm{H}^{+}, 288.1422\right.$; found, 288.1418.

(E)-Phenyl(prop-2-yn-1-ylimino)(2-(pyridin-2-yl)vinyl)- $\lambda$-sulfanone (19d). The title compound was prepared according to General Procedure J employing sulfoximine $\mathbf{5 d}$ ( $40 \mathrm{mg}, 0.16 \mathrm{mmol}$ ). Purification by flash column chromatography (KP-Sil, $0 \%$ grading to $100 \%$ EtOAc/Pentane, eluted at 56\% EtOAc) afforded 19d (31 mg, 77\%) as a brown oil; $\mathrm{R}_{f}=0.30$ (50\% EtOAc/Pentane); IR (film)/cm-1 3298,3056 , 2926, 1580, 1267, 1215, 1129, 1080, 969, 752, 685; $\left.{ }^{1} \mathrm{H} \mathrm{NMR} \mathrm{(400} \mathrm{MHz,} \mathrm{CDCl}\right)_{3} \delta 8.60-8.59(\mathrm{~d}, J=5.5 \mathrm{~Hz}, 1 \mathrm{H}), 8.02-8.00(\mathrm{~m}, 2 \mathrm{H}), 7.73-7.69$ (m, 1H), $7.59(\mathrm{~d}, J=15.1 \mathrm{~Hz}, 1 \mathrm{H}), 7.61-7.57(\mathrm{~m}, 1 \mathrm{H}), 7.56-7.51(\mathrm{~m}, 2 \mathrm{H}), 7.40(\mathrm{~d}, J=15.0 \mathrm{~Hz}, 1 \mathrm{H}), 7.38(\mathrm{~d}, J=7.4 \mathrm{~Hz}, 1 \mathrm{H}), 7.28-7.25(\mathrm{~m}, 1 \mathrm{H})$; ${ }^{13} \mathrm{C}\left\{{ }^{1} \mathrm{H}\right\}$ NMR $\left(101 \mathrm{~Hz}, \mathrm{CDCl}_{3}\right) \delta 151.4,150.2,141.3,138.9,137.0,133.1,131.8,129.4(2 \times \mathrm{C}), 128.8(2 \times \mathrm{C}), 125.1,124.8,83.1,70.5,32.7$ HRMS (ESI-TOF) $m / z$ Calcd for $\mathrm{C}_{16} \mathrm{H}_{15} \mathrm{~N}_{2} \mathrm{OS}^{+}[\mathrm{M}+\mathrm{H}]^{+}, 283.0905$; found, 283.0920 .

4-Phenyl-1-( $N$-(prop-2-yn-1-yl)vinylsulfonimidoyl)piperidine (19e). The title compound was prepared according to General Procedure J employing sulfonimidamide $18 a(16 \mathrm{mg}, 0.07 \mathrm{mmol}$ ). Purification by flash column chromatography (KP-Sil, $35 \%$ grading to $100 \%$ EtOAc in pentane, eluted at 60\%) afforded sulfonimidamide $19 \mathrm{e}(10 \mathrm{mg}, 52 \%)$ as a colourless oil; $\mathrm{R}_{f}=0.70\left(100 \%\right.$ EtOAc); IR (film)/ $\mathrm{cm}^{-1} 3284,2918$, 
2844, 1278, 1230, 1140, 924, 756; ${ }^{\mathrm{H}} \mathrm{NMR}\left(400 \mathrm{MHz}, \mathrm{CDCl}_{3}\right) \delta$ 7.36-7.28 (m, 2H), 7.25-7.16 (m, 3H), $6.52(\mathrm{dd}, J=16.7,9.9 \mathrm{~Hz}, 1 \mathrm{H}), 6.24(\mathrm{~d}$, $J=16.8 \mathrm{~Hz}, 1 \mathrm{H}), 6.01(\mathrm{~d}, J=10.0 \mathrm{~Hz}, 1 \mathrm{H}), 4.12-3.75(\mathrm{~m}, 4 \mathrm{H}), 2.72-2.47(\mathrm{~m}, 3 \mathrm{H}), 2.22(\mathrm{t}, J=2.6 \mathrm{~Hz}, 1 \mathrm{H}), 2.02-1.69(\mathrm{~m}, 4 \mathrm{H}) ;{ }^{13} \mathrm{C}\{1 \mathrm{H}\} \mathrm{NMR}(101$ $\left.\mathrm{Hz}, \mathrm{CDCl}_{3}\right) \delta 145.2,132.9,128.7(2 \times \mathrm{C}), 127.4,126.9(2 \times \mathrm{C}), 126.7,83.0,70.1,47.6,47.5,42.2,33.1,33.0$, 30.7; HRMS (ESI-TOF) $\mathrm{m} / z \mathrm{Calcd}$ for $\mathrm{C}_{16} \mathrm{H}_{21} \mathrm{~N}_{2} \mathrm{OS}+[\mathrm{M}+\mathrm{H}]^{+}, 289.1375$; found, 289.1371 .

4-Methyl-2-phenyl-1-(N-(prop-2-yn-1-yl)vinylsulfonimidoyl)piperazine (19f). The title compound was prepared according to General Procedure J employing sulfonimidamide $\mathbf{1 8 b}\left(53 \mathrm{mg}, 0.20 \mathrm{mmol}\right.$, dr 1:2). Purification by flash column chromatography $\left(\mathrm{SiO}_{2}, 10 \%\right.$ acetone in pentane) afforded sulfonimidamide $19 \mathrm{f}(29 \mathrm{mg}, 48 \%, \mathrm{dr} 1: 2)$ as a brown oil; $\mathrm{R}_{f}=0.26\left(10 \%\right.$ acetone in pentane); IR (film)/cm ${ }^{-1} 3287$, $2937,2844,2795,1457,1278,1230,1148,972,917,767 ;{ }^{1} \mathrm{H} \mathrm{NMR}\left(400 \mathrm{MHz}, \mathrm{CDCl}_{3}\right) \delta 7.77-7.72(\mathrm{~m}, 2 \mathrm{H}), 7.70(\mathrm{dd}, J=7.4,1.8 \mathrm{~Hz}, 2 \mathrm{H}), 7.37-$ $7.24(\mathrm{~m}, 6 \mathrm{H}), 6.29(\mathrm{dd}, J=16.5,9.5 \mathrm{~Hz}, 1 \mathrm{H}), 6.16(\mathrm{~d}, J=16.5 \mathrm{~Hz}, 1 \mathrm{H}), 6.00(\mathrm{dd}, J=16.5,9.6 \mathrm{~Hz}, 1 \mathrm{H}), 5.89(\mathrm{~d}, J=16.5 \mathrm{~Hz}, 1 \mathrm{H}), 5.77(\mathrm{~d}, J=9.6$ $\mathrm{Hz}, 1 \mathrm{H}), 5.56(\mathrm{~d}, J=9.6 \mathrm{~Hz}, 1 \mathrm{H}), 4.98(\mathrm{~d}, J=4.2 \mathrm{~Hz}, 1 \mathrm{H}), 4.94-4.90(\mathrm{~m}, 1 \mathrm{H}), 3.89-3.85(\mathrm{~m}, 4 \mathrm{H}), 3.66-3.57(\mathrm{~m}, 2 \mathrm{H}), 3.29-3.22(\mathrm{~m}, 2 \mathrm{H}), 3.15(\mathrm{dt}$, $J=11.9,1.8 \mathrm{~Hz}, 1 \mathrm{H}), 3.10(\mathrm{dt}, J=11.6,1.8 \mathrm{~Hz}, 1 \mathrm{H}), 2.91-2.87(\mathrm{~m}, 1 \mathrm{H}), 2.81-2.77(\mathrm{~m}, 1 \mathrm{H}), 2.56(\mathrm{dd}, J=11.6,4.5 \mathrm{~Hz}, 1 \mathrm{H}), 2.53-2.44(\mathrm{~m}, 1 \mathrm{H})$, 2.31-2.27 (m, 8H), $2.21(\mathrm{t}, J=2.5 \mathrm{~Hz}, 1 \mathrm{H}), 2.19(\mathrm{t}, J=2.5 \mathrm{~Hz}, 1 \mathrm{H}) ;{ }^{13} \mathrm{C}\left\{{ }^{1} \mathrm{H}\right\} \mathrm{NMR}\left(101 \mathrm{~Hz}, \mathrm{CDCl}_{3}\right) \delta 139.3,136.8,134.3,129.3,128.5,128.2$, 128.0, 127.7, 126.1, 124.8, 82.9, 82.6, 70.2, 69.9, 59.2, 57.2, 56.2, 55.9, 54.8, 54.7, 46.2, 41.9, 41.5, 30.8, 30.7; HRMS (ESI-TOF) m/z Calcd for $\mathrm{C}_{16} \mathrm{H}_{22} \mathrm{~N}_{3} \mathrm{OS}+[\mathrm{M}+\mathrm{H}]+, 304.1484$; found, 304.1497.

$N$-(3-(10,11-Dihydro-5H-dibenzo[a,d] [7] annulen-5-ylidene)propyl)- $N$-methyl- $N$ '-(prop-2-yn-1-yl)ethenesulfonimidamide (19g). The title compound was prepared according to General Procedure J employing sulfonimidamide 18c (70 mg, $0.20 \mathrm{mmol})$. Purification by flash column chromatography ( $\mathrm{SiO}_{2}, 30 \%$ EtOAc in pentane) afforded sulfonimidamide $\mathbf{1 9 g}(63 \mathrm{mg}, 81 \%)$ as a brown oil; $\mathrm{R}_{f}=0.53(50 \%$ EtOAc in

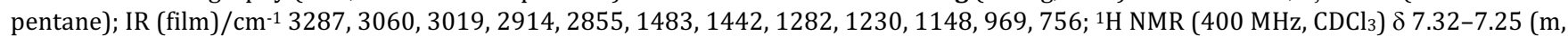
$1 \mathrm{H}), 7.26-7.09(\mathrm{~m}, 6 \mathrm{H}), 7.08-7.02(\mathrm{~m}, 1 \mathrm{H}), 6.49-6.38(\mathrm{~m}, 1 \mathrm{H}), 6.15(\mathrm{~d}, J=16.7 \mathrm{~Hz}, 1 \mathrm{H}), 5.92-5.81(\mathrm{~m}, 2 \mathrm{H}), 3.82(\mathrm{~d}, J=2.9 \mathrm{~Hz}, 2 \mathrm{H}), 3.51-3.11$ $(\mathrm{m}, 5 \mathrm{H}), 3.03-2.93(\mathrm{~m}, 1 \mathrm{H}), 2.87-2.74(\mathrm{~m}, 1 \mathrm{H}), 2.69(\mathrm{~s}, 3 \mathrm{H}), 2.41(\mathrm{qd}, J=7.3,1.4 \mathrm{~Hz}, 2 \mathrm{H}) ;{ }^{3} \mathrm{C}\left\{{ }^{1} \mathrm{H}\right\} \mathrm{NMR}\left(101 \mathrm{~Hz}, \mathrm{CDCl}_{3}\right) \delta 145.0,140.8,139.7$, 139.3, 137.0, 133.1, 130.0, 128.5, 128.1, 128.0, 127.6, 127.2 (2 × C), 126.4, 126.1, 125.8, 82.7, 70.0, 50.4 (2 × C), 33.7, 32.0, 30.6, 28.1; HRMS (ESI-TOF) $\mathrm{m} / \mathrm{z}$ Calcd for $\mathrm{C}_{24} \mathrm{H}_{27} \mathrm{~N}_{2} \mathrm{OS}+[\mathrm{M}+\mathrm{H}]^{+}, 391.1844$; found, 391.1854 .

Chiral separation of $\mathbf{1 0 g}$. Vinyl sulfoximine $\mathbf{1 0 g}(68 \mathrm{mg}$ ) was dissolved to $12 \mathrm{mg} / \mathrm{mL}$ in $\mathrm{MeOH}$ and was then purified by HPLC using a Chiralpak IG $(20 \mathrm{~mm} \times 250 \mathrm{~mm}, 5 \mu \mathrm{m})$ column. Combined fractions of each isomer were evaporated to dryness to afford $\mathbf{1 0 g}-\mathbf{A}(12.9 \mathrm{mg}$, $>99 \% \mathrm{de},), \mathbf{1 0 g}-\mathbf{B}(10.2 \mathrm{mg}, 97 \% \mathrm{de})$ as yellow oils.

10g-A; IR (film)/ $\mathrm{cm}^{-1} 3295,3060,2926,1495,1446,1222,753 ;{ }^{1} \mathrm{H} \mathrm{NMR}\left(400 \mathrm{MHz}, \mathrm{CDCl}_{3}\right) \delta 8.00-7.96(\mathrm{~m}, 2 \mathrm{H}), 7.61-7.52(\mathrm{~m}, 3 \mathrm{H}), 7.28-$ $7.09(\mathrm{~m}, 5 \mathrm{H}), 6.93(\mathrm{dd}, J=14.9,5.8 \mathrm{~Hz}, 1 \mathrm{H}), 6.56(\mathrm{dd}, J=14.9,1.4 \mathrm{~Hz}, 1 \mathrm{H}), 3.60-3.51(\mathrm{~m}, 1 \mathrm{H}), 2.73-2.63(\mathrm{~m}, 2 \mathrm{H}), 2.58(\mathrm{~s}, 1 \mathrm{H}), 1.88-1.77(\mathrm{~m}$, 2H), $1.66(\mathrm{~s}, 2 \mathrm{H}) ;{ }^{13} \mathrm{C}\left\{{ }^{1} \mathrm{H}\right\} \mathrm{NMR}\left(101 \mathrm{~Hz}, \mathrm{CDCl}_{3}\right) \delta 148.7,142.9,141.2,133.0,131.8,129.3(2 \times \mathrm{C}), 128.7(2 \times \mathrm{C}), 128.5(2 \times \mathrm{C}), 128.1(2 \times \mathrm{C})$, $126.3,51.7,38.4,32.2$.

10g-B; IR (film)/ $\mathrm{cm}^{-1} 3306,3063,2922,1495,1446,1222,987,752,{ }^{1}{ }^{H}$ NMR $\left(400 \mathrm{MHz}, \mathrm{CDCl}_{3}\right) \delta 8.01-7.96(\mathrm{~m}, 2 \mathrm{H}), 7.62-7.52(\mathrm{~m}, 3 \mathrm{H})$, 7.28-7.10 (m, 5H), $6.93(\mathrm{dd}, J=14.9,5.8 \mathrm{~Hz}, 1 \mathrm{H}), 6.55(\mathrm{dd}, J=14.9,1.4 \mathrm{~Hz}, 1 \mathrm{H}), 3.60-3.47(\mathrm{~m}, 1 \mathrm{H}), 2.74-2.63(\mathrm{~m}, 2 \mathrm{H}), 2.55(\mathrm{~s}, 1 \mathrm{H}), 1.91-1.78$ $(\mathrm{m}, 2 \mathrm{H}), 1.66(\mathrm{~s}, 2 \mathrm{H}) ;{ }^{13} \mathrm{C}\left\{{ }^{1} \mathrm{H}\right\}$ NMR $\left(101 \mathrm{~Hz}, \mathrm{CDCl}_{3}\right) \delta 148.9,143.0,141.1,133.0,131.8,129.4(2 \times \mathrm{C}), 128.7(2 \times \mathrm{C}), 128.5(2 \times \mathrm{C}), 128.1(2 \times$ C), $126.3,51.7,38.5,32.12$.

Chiral separation of 19f. Vinyl sulfonimidamide $19 f(21.8 \mathrm{mg})$ was dissolved to $10 \mathrm{mg} / \mathrm{mL}$ in Heptane:EtOH 6:4 and was then purified by HPLC using a Amy-C $(20 \mathrm{~mm} \times 250 \mathrm{~mm}, 5 \mu \mathrm{m})$ column. Combined fractions of each isomer were evaporated to dryness to afford 19f-A (2.5 $\mathrm{mg},>99 \%$ ee, $>99 \%$ de, $\left.[\alpha]_{D}^{21}=+28\left(c 0.003, \mathrm{CHCl}_{3}\right)\right), \mathbf{1 9 f - B}\left(4.4 \mathrm{mg},>99 \%\right.$ ee, $\left.>99 \% \mathrm{de},[\alpha]_{D}^{21}-37(c 0.002, \mathrm{CHCl})\right), \mathbf{1 9 f}-\mathrm{C}(1.9 \mathrm{mg}, 96 \%$ ee, $>99 \%$ de, $\left.[\alpha]_{D}^{21}+31\left(c 0.001, \mathrm{CHCl}_{3}\right)\right)$ and 19f-D $\left(3.6 \mathrm{mg},>99 \%\right.$ ee, $97 \%$ de, $\left.\left.[\alpha]_{D}^{21}-28\left(c 0.003, \mathrm{CHCl}_{3}\right)\right)\right)$ as clear glassy solids.

Enantiomeric pair 19f-A and 19f-D; IR (film) $\left./ \mathrm{cm}^{-1} 3287,2937,2795,1457,1282,1140,972,917 ;{ }^{1} \mathrm{H} \mathrm{NMR} \mathrm{(400} \mathrm{MHz,} \mathrm{CDCl} 3\right) \delta 7.75(\mathrm{dd}, J$ $=8.0,1.7 \mathrm{~Hz}, 2 \mathrm{H}), 7.40-7.27(\mathrm{~m}, 3 \mathrm{H}), 6.00(\mathrm{dd}, J=16.5,9.6 \mathrm{~Hz}, 1 \mathrm{H}), 5.89(\mathrm{~d}, J=16.5 \mathrm{~Hz}, 1 \mathrm{H}), 5.55(\mathrm{~d}, J=9.5 \mathrm{~Hz}, 1 \mathrm{H}), 4.91(\mathrm{~d}, J=4.3 \mathrm{~Hz}, 1 \mathrm{H})$, $3.86(\mathrm{~d}, J=2.5 \mathrm{~Hz}, 2 \mathrm{H}), 3.57(\mathrm{~s}, 1 \mathrm{H}), 3.24(\mathrm{~d}, J=3.3 \mathrm{~Hz}, 1 \mathrm{H}), 3.09(\mathrm{~d}, J=11.6 \mathrm{~Hz}, 1 \mathrm{H}), 2.94-2.82(\mathrm{~m}, 1 \mathrm{H}), 2.55(\mathrm{dd}, J=11.7,4.5 \mathrm{~Hz}, 1 \mathrm{H}), 2.28(\mathrm{~s}$, 3H), $2.27(\mathrm{~m}, 1 \mathrm{H}), 2.19(\mathrm{t}, J=2.5 \mathrm{~Hz}, 1 \mathrm{H}) ;{ }^{13} \mathrm{C}\left\{{ }^{1} \mathrm{H}\right\} \mathrm{NMR}\left(101 \mathrm{~Hz}, \mathrm{CDCl}_{3}\right) \delta 139.6,134.6,129.5,128.2,127.8,126.2,83.1,70.1,59.5,56.5,55.0$, $46.5,41.7,30.9$.

Enantiomeric pair 19f-B and 19f-C; IR (film)/ $\mathrm{cm}^{-1} 3287,2939,2795,1454,1278,1230,1151,976,928 ;{ }^{1} \mathrm{H} \mathrm{NMR}\left(400 \mathrm{MHz}, \mathrm{CDCl}_{3}\right) \delta 7.75-$ $7.62(\mathrm{~m}, 2 \mathrm{H}), 7.38-7.27(\mathrm{~m}, 3 \mathrm{H}), 6.28(\mathrm{dd}, J=16.6,9.5 \mathrm{~Hz}, 1 \mathrm{H}), 6.16(\mathrm{~d}, J=16.6 \mathrm{~Hz}, 1 \mathrm{H}), 5.76(\mathrm{~d}, J=9.5 \mathrm{~Hz}, 1 \mathrm{H}), 4.98(\mathrm{~d}, J=4.3 \mathrm{~Hz}, 1 \mathrm{H}), 3.86$ (d, $J=2.5 \mathrm{~Hz}, 2 \mathrm{H}), 3.71-3.52(\mathrm{~m}, 1 \mathrm{H}), 3.31-3.04(\mathrm{~m}, 2 \mathrm{H}), 2.85-2.71(\mathrm{~m}, 1 \mathrm{H}), 2.45(\mathrm{dd}, J=11.9,4.4 \mathrm{~Hz}, 1 \mathrm{H}), 2.28(\mathrm{~s}, 3 \mathrm{H}), 2.22-2.12(\mathrm{~m}, 2 \mathrm{H})$; ${ }^{13} \mathrm{C}\left\{{ }^{1} \mathrm{H}\right\}$ NMR $\left(101 \mathrm{~Hz}, \mathrm{CDCl}_{3}\right) \delta 140.5,137.1,128.7,128.4,127.6,124.9,82.5,70.4,57.4,56.1,54.9,46.5,42.1,31.0$.

Chiral separation of $19 \mathrm{~g}$. Vinyl sulfonimidamide $19 \mathrm{~g}(51.6 \mathrm{mg})$ was dissolved to $16 \mathrm{mg} / \mathrm{mL}$ in 2:1 IPA: $\mathrm{CH}_{2} \mathrm{Cl}_{2}$ and was then purified by HPLC using a Chiralpak IG $(20 \mathrm{~mm} \times 250 \mathrm{~mm}, 5 \mu \mathrm{m})$ column. Combined fractions of each enantiomer were evaporated to dryness to afford 19g-A $\left(10.6 \mathrm{mg}, 99 \%\right.$ ee, $\left.[\alpha]_{D}^{21}-33\left(c 0.01, \mathrm{CHCl}_{3}\right)\right)$ and $\mathbf{1 9 g}-\mathbf{B}\left(13.3 \mathrm{mg}, 98 \%\right.$ ee, $\left.[\alpha]_{D}^{21}+33\left(c 0.01, \mathrm{CHCl}_{3}\right)\right)$ as brown glassy solids.

\section{Computational methods}

All calculations were performed using Gaussian $16 .^{62}$ Stationary points were confirmed using frequency calculations (default divergence criteria). Conformational searches were carried out as described in the Supporting Information. Electronic Circular Dichroism spectra were calculated for individual conformers using TDDFT calculations at cam-B3LYP/6-311++g(d,p) in a continuum solvent (CPCM) acetonitrile for up to 200 states. Copies of Gaussian log files for optimisations and frequency calculations, TDDFT calculations, and tables of ECD data are available from a data depository via the dataset collection DOIs $10.14469 / \mathrm{hpc} / 7859$ (sulfoximines) and $\underline{10.14469 / \mathrm{hpc} / 7577}$ (sulfonimidamides) and datasets cited therein.

\section{Electronic circular dichroism}

The circular dichroism and UV-vis spectra were recorded on a Chirascan spectrometer (Applied Photophysics). A $0.1 \mathrm{~cm}$ path-length cuvette was loaded with $200 \mu \mathrm{L}$ each compound ( $50 \mu \mathrm{M}$ in acetonitrile) or acetonitrile blank. The elipticity was measured from $340-195 \mathrm{~nm}$ in $1.0 \mathrm{~nm}$ intervals (bandwidth $=2.0 \mathrm{~nm}$ ) for $1.0 \mathrm{~s}$ per measurement. Measurements were taken in duplicate and normalised against the acetonitrile control.

\section{Configurational assignment by comparison of the calculated and experimental ECD}

Comparative analysis was performed using SpecDis. ${ }^{44}$ For each structure/sub-structure, the computed ECD and UV-vis spectra were summed according to their Boltzmann weighting at $298 \mathrm{~K}$. The relevant experimental ECD and UV-vis spectra were then input and similarity analysis performed by first aligning the UV spectra (shift range $=-30$ to $+30 \mathrm{~nm}$; bandwidth $=0.1$ to 0.3 ; energy range $=200$ to $340 \mathrm{~nm}$ ) and then performing a local refinement to optimize the alignment of the ECD. Assignments were made on the basis of both manual inspection of the ECD alignments and the quantification of the similarity scores.

\section{ASSOCIATED CONTENT}




\section{Supporting Information}

Supplementary Information (PDF) contains Supplementary Figures and Supplementary Data. Supplementary Figures S1-S7 detail the relative energies and structures of conformers of $10 \mathrm{~g}, 19 \mathrm{f}$ and $19 \mathrm{~g}$ and the full comparisons of the computational and experimental UV and ECD spectra. Supplementary Data contains ${ }^{1} \mathrm{H}$ and ${ }^{13} \mathrm{C}$ NMR spectra for novel compounds, HPLC traces of enantioenriched compounds and computational data including Supplementary Tables S1-S7.

\section{AUTHOR INFORMATION}

\section{Corresponding Author}

* Gregory B. Craven - School of Molecular and Cellular Biology, The Astbury Centre for Structural Molecular Biology, University of Leeds, LS2 9JT, UK; orcid.org/0000-0003-3414-8348; Email: g.craven13@gmail.com;

* James A. Bull - Department of Chemistry, Imperial College London, Molecular Sciences Research Hub, White City Campus, Wood Lane, London, W12 0BZ, U.K; orcid.org/0000-0003-3993-5818; Email: j.bull@imperial.ac.uk;

* Alan Armstrong - Department of Chemistry, Imperial College London, Molecular Sciences Research Hub, White City Campus, Wood Lane, London, W12 0BZ, U.K; orcid.org/0000-0002-3692-3099; Email: a.armstrong@imperial.ac.uk.

\section{Author Contributions}

All authors have given approval to the final version of the manuscript.

\section{Funding Sources}

We gratefully acknowledge EPSRC for funding (studentships to ELB and SG, and doctoral prize fellowship to GBC), and The Royal Society [University Research Fellowship (UF140161, to JAB) and Research Grants (RG150444 and RGF\EA\180031)]. This work was additionally supported by grants from the Institute of Chemical Biology (Imperial College London) and EPSRC (Studentship award EP/F500416/1). The circular dichroism facility at University of Leeds was funded by the Wellcome Trust (094232).

\section{ACKNOWLEDGMENT}

We thank Reach Separations (Bio City, NG1 1GF, UK) for supporting this work by conducting preparatory and analytical chiral HPLC of compounds 10g, 19f and 19g. We are grateful to Prof Henry Rzepa (Imperial College) for invaluable advice and discussions on CD spectra calculations.

\section{ABBREVIATIONS}

BTK, Bruton's tyrosine kinase; Cdk2, cyclin-dependent kinase 2; DFT, density functional theory; DMPK, drug metabolism and pharmacokinetics; ECD, electronic circular dichroism; ERK2, mitogen-activated protein kinase 1; HPLC, high-performance liquid chromatography; HWE, Horner-Wadsworth-Emmons; Nrf2, nuclear factor erythroid 2-related factor 2; SFC, supercritical fluid chromatography; USP7, ubiquitin specific protease 7;

\section{REFERENCES}

(1) a) Lücking, U. Sulfoximines: A Neglected Opportunity in Medicinal Chemistry. Angew. Chem. Int. Ed. 2013, 52, 9399-9408. b) Lücking, U. Neglected Sulfur(vi) Pharmacophores in Drug Discovery: Exploration of Novel Chemical Space by the Interplay of Drug Design and Method Development. Org. Chem. Front. 2019, 6, 1319-1324.

(2) Chinthakindi, P. K.; Naicker, T.; Thota, N.; Govender, T.; Kruger, H. G.; Arvidsson, P. I. Sulfonimidamides in Medicinal and Agricultural Chemistry. Angew. Chem. Int. Ed. 2017, 56, 4100-4109.

(3) Rowe, W. B.; Meister, A. Identification of L-Methionine-S-Sulfoximine as the Convulsant Isomer of Methionine Sulfoximine. Proc. Natl. Acad. Sci. 1970, 66, 500-506.

(4) a) Sirvent, J. A.; Lücking, U. Novel Pieces for the Emerging Picture of Sulfoximines in Drug Discovery: Synthesis and Evaluation of Sulfoximine Analogues of Marketed Drugs and Advanced Clinical Candidates. ChemMedChem 2017, 12, 487-501. b) Frings, M.; Bolm, C.; Blum, A.; Gnamm, C. Sulfoximines from a Medicinal Chemist's Perspective: Physicochemical and in Vitro Parameters Relevant for Drug Discovery. Eur. J. Med. Chem. 2017, 126, 225-245. c) Wiezorek, S.; Lamers, P.; Bolm, C. Conversion and Degradation Pathways of Sulfoximines. Chem. Soc. Rev. 2019, 48, 5408-5423. d) Mäder, P.; Kattner, L. Sulfoximines as Rising Stars in Modern Drug Discovery? Current Status and Perspective on an Emerging Functional Group in Medicinal Chemistry. J. Med. Chem. 2020, 63, 14243-14275.

(5) a) Lücking, U.; Jautelat, R.; Krüger, M.; Brumby, T.; Lienau, P.; Schäfer, M.; Briem, H.; Schulze, J.; Hillisch, A.; Reichel, A.; et al. The Lab Oddity Prevails: Discovery of Pan-CDK Inhibitor (R)-S-Cyclopropyl-S-(4-\{[4-\{[(1R,2R)-2-Hydroxy-1-Methylpropyl]0xy\}-5-(Trifluoromethyl)Pyrimidin-2-Yl]Amino\}phenyl)Sulfoximide (BAY 1000394) for the Treatment of Cancer. ChemMedChem 2013, 8, 1067-1085. b) Foote, K. M.; Nissink, J. W. M.; McGuire, T.; Turner, P.; Guichard, S.; Yates, J. W. T.; Lau, A.; Blades, K.; Heathcote, D.; Odedra, R.; et al. Discovery and Characterization of AZD6738, a Potent Inhibitor of Ataxia Telangiectasia Mutated and Rad3 Related (ATR) Kinase with Application as an Anticancer Agent. J. Med. Chem. 2018, 61, 9889-9907. c) Lücking, U.; Scholz, A.; Lienau, P.; Siemeister, G.; Kosemund, D.; Bohlmann, R.; Briem, H.; Terebesi, I.; Meyer, K.; Prelle, K.; Denner, K.; Bömer, U.; Schäfer, M.; Eis, K.; Valencia, R.; Ince, S.; von Nussbaum, F.; Mumberg, D.; Ziegelbauer, K.; Klebl, B.; Choidas, A.; Nussbaumer, P.; Baumann, M.; Schultz-Fademrecht, C.; Rühter, G.; Eickhoff, J.; Brands, M. Identification of Atuveciclib (BAY 1143572), the First Highly Selective, Clinical PTEFb/CDK9 Inhibitor for the Treatment of Cancer. ChemMedChem 2017, 12, 1776-1793.

(6) a) Palmer, J. T.; Rasnick, D.; Klaus, J. L.; Bromme, D. Vinyl Sulfones as Mechanism-Based Cysteine Protease Inhibitors. J. Med. Chem. 1995, 38, 3193-3196. b) Roush, W. R.; Gwaltney, S. L.; Cheng, J.; Scheidt, K. A.; McKerrow, J. H.; Hansell, E. Vinyl Sulfonate Esters and Vinyl Sulfonamides: Potent, Irreversible Inhibitors of Cysteine Proteases. J. Am. Chem. Soc. 1998, 120, 10994-10995. c) Kerr, I. D.; Lee, J. H.; Farady, C. J.; Marion, R.; Rickert, M.; Sajid, M.; Pandey, K. C.; Caffrey, C. R.; Legac, J.; Hansell, E.; McKerrow, J. H.; Craik, C. S.; Rosenthal, P. J.; Brinen, L. S. Vinyl Sulfones as Antiparasitic Agents and a Structural Basis for Drug Design. J. Biol. Chem. 2009, 284, 25697-25703. d) Pakavathkumar, P.; Noël, A.; Lecrux, C.; Tubeleviciute-Aydin, A.; Hamel, E.; Ahlfors, J.-E.; LeBlanc, A. C. Caspase Vinyl Sulfone Small Molecule 
Inhibitors Prevent Axonal Degeneration in Human Neurons and Reverse Cognitive Impairment in Caspase-6-Overexpressing Mice. Mol. Neurodegener. 2017, 12, 22.

(7) Jacobsen, W.; Christians, U.; Benet, L. Z. In Vitro Evaluation of the Disposition of a Novel Cysteine Protease Inhibitor. Drug Metab. Dispos. 2000, 28, 1343-1351.

(8) Anscombe, E.; Meschini, E.; Mora-Vidal, R.; Martin, M. P.; Staunton, D.; Geitmann, M.; Danielson, U. H.; Stanley, W. A.; Wang, L. Z.; Reuillon, T.; Golding, B. T.; Cano, C.; Newell, D. R.; Noble, M. E. M.; Wedge, S. R.; Endicott, J. A.; Griffin, R. J. Identification and Characterization of an Irreversible Inhibitor of CDK2. Chem. Biol. 2015, 22, 1159-1164.

(9) Ward, R. A.; Colclough, N.; Challinor, M.; Debreczeni, J. E.; Eckersley, K.; Fairley, G.; Feron, L.; Flemington, V.; Graham, M. A.; Greenwood, R.; Hopcroft, P.; Howard, T. D.; James, M.; Jones, C. D.; Jones, C. R.; Renshaw, J.; Roberts, K.; Snow, L.; Tonge, M.; Yeung, K. StructureGuided Design of Highly Selective and Potent Covalent Inhibitors of ERK1/2. J. Med. Chem. 2015, 58, 4790-4801.

(10) Ostrem, J. M.; Peters, U.; Sos, M. L.; Wells, J. A.; Shokat, K. M. K-Ras(G12C) Inhibitors Allosterically Control GTP Affinity and Effector Interactions. Nature 2013, 503, 548-551.

(11) Li, X.; Zuo, Y.; Tang, G.; Wang, Y.; Zhou, Y.; Wang, X.; Guo, T.; Xia, M.; Ding, N.; Pan, Z. Discovery of a Series of 2,5-Diaminopyrimidine Covalent Irreversible Inhibitors of Bruton's Tyrosine Kinase with in Vivo Antitumor Activity. J. Med. Chem. 2014, 57, 5112-5128.

(12) Turnbull, A. P.; Ioannidis, S.; Krajewski, W. W.; Pinto-Fernandez, A.; Heride, C.; Martin, A. C. L.; Tonkin, L. M.; Townsend, E. C.; Buker, S. M.; Lancia, D. R.; Caravella, J. A.; Toms, A. V; Charlton, T. M.; Lahdenranta, J.; Wilker, E.; Follows, B. C.; Evans, N. J.; Stead, L.; Alli, C.; Zarayskiy, V. V; Talbot, A. C.; Buckmelter, A. J.; Wang, M.; McKinnon, C. L.; Saab, F.; McGouran, J. F.; Century, H.; Gersch, M.; Pittman, M. S.; Marshall, C. G.; Raynham, T. M.; Simcox, M.; Stewart, L. M. D.; McLoughlin, S. B.; Escobedo, J. A.; Bair, K. W.; Dinsmore, C. J.; Hammonds, T. R.; Kim, S.; Urbé, S.; Clague, M. J.; Kessler, B. M.; Komander, D. Molecular Basis of USP7 Inhibition by Selective Small-Molecule Inhibitors. Nature 2017, 550, 481-486.

(13) a) Fang, Y.; Luo, Z.; Xu, X. Recent Advances in the Synthesis of Vinyl Sulfones. RSC Adv. 2016, 6, 59661-59676. b) Reuter, D. C.; McIntosh, J. E.; Guinn, A. C.; Madera, A. M. Synthesis of Vinyl Sulfonamides Using the Horner Reaction. Synthesis. 2003, 2321-2324. c) Roush, W. R.; Cheng, J.; Knapp-Reed, B.; Alvarez-Hernandez, A.; McKerrow, J. H.; Hansell, E.; Engel, J. C. Potent Second Generation Vinyl Sulfonamide Inhibitors of the Trypanosomal Cysteine Protease Cruzain. Bioorg. Med. Chem. Lett. 2001, 11, 2759-2762. d) Craven, G. B.; Affron, D. P.; Raymond, P. N.; Mann, D. J.; Armstrong, A. Vinyl Sulfonamide Synthesis for Irreversible Tethering via a Novel $\alpha$-Selenoether Protection Strategy. Medchemcomm 2019, 10, 158-163. e) Kiemele, E. R.; Wathier, M.; Bichler, P.; Love, J. A. Total Synthesis of K777: Successful Application of Transition-Metal-Catalyzed Alkyne Hydrothiolation toward the Modular Synthesis of a Potent Cysteine Protease Inhibitor. Org. Lett. 2016, 18, 492-495.

(14) a) Jones, L. H. Reactive Chemical Probes: Beyond the Kinase Cysteinome. Angew. Chem. Int. Ed. 2018, 57, 9220-9223. b) Gehringer, M.; Laufer, S. A. Emerging and Re-Emerging Warheads for Targeted Covalent Inhibitors: Applications in Medicinal Chemistry and Chemical Biology. J. Med. Chem. 2019, 62, 5673-5724. c) Sutanto, F.; Konstantinidou, M.; Dömling, A. Covalent Inhibitors: A Rational Approach to Drug Discovery. RSC Med. Chem. 2020, 11, 876-884.

(15) Cisar, J. S.; Cravatt, B. F. Fully Functionalized Small-Molecule Probes for Integrated Phenotypic Screening and Target Identification. J. Am. Chem. Soc. 2012, 134, 10385-10388.

(16) Wang, Y.; Dix, M. M.; Bianco, G.; Remsberg, J. R.; Lee, H.-Y.; Kalocsay, M.; Gygi, S. P.; Forli, S.; Vite, G.; Lawrence, R. M.; Parker, C. G.; Cravatt, B. F. Expedited Mapping of the Ligandable Proteome Using Fully Functionalized Enantiomeric Probe Pairs. Nat. Chem. 2019, 11, 1113-1123.

(17) a) Nonoo, R. H.; Armstrong, A.; Mann, D. J. Kinetic Template-Guided Tethering of Fragments. ChemMedChem 2012, 7, $2082-2086$. b) Kathman, S. G.; Xu, Z.; Statsyuk, A. V. A Fragment-Based Method to Discover Irreversible Covalent Inhibitors of Cysteine Proteases. J. Med. Chem. 2014, 57, 4969-4974. c) Craven, G. B.; Affron, D. P.; Allen, C. E.; Matthies, S.; Greener, J. G.; Morgan, R. M. L.; Tate, E. W.; Armstrong, A.; Mann, D. J. High-Throughput Kinetic Analysis for Target-Directed Covalent Ligand Discovery. Angew. Chem. Int. Ed. 2018, 57, 5257-5261. d) Resnick, E.; Bradley, A.; Gan, J.; Douangamath, A.; Krojer, T.; Sethi, R.; Geurink, P. P.; Aimon, A.; Amitai, G.; Bellini, D.; Bennett, J.; Fairhead, M.; Fedorov, O.; Gabizon, R.; Gan, J.; Guo, J.; Plotnikov, A.; Reznik, N.; Ruda, G. F.; Díaz-Sáez, L.; Straub, V. M.; Szommer, T.; Velupillai, S.; Zaidman, D.; Zhang, Y.; Coker, A. R.; Dowson, C. G.; Barr, H. M.; Wang, C.; Huber, K. V. M.; Brennan, P. E.; Ovaa, H.; von Delft, F.; London, N. Rapid Covalent-Probe Discovery by Electrophile-Fragment Screening. J. Am. Chem. Soc. 2019, 141, 8951-8968. e) Douangamath, A.; Fearon, D.; Gehrtz, P.; Krojer, T.; Lukacik, P.; Owen, C. D.; Resnick, E.; Strain-Damerell, C.; Aimon, A.; Ábrányi-Balogh, P.; BrandãoNeto, J.; Carbery, A.; Davison, G.; Dias, A.; Downes, T. D.; Dunnett, L.; Fairhead, M.; Firth, J. D.; Jones, S. P.; Keeley, A.; Keserü, G. M.; Klein, H. F.; Martin, M. P.; Noble, M. E. M.; O’Brien, P.; Powell, A.; Reddi, R. N.; Skyner, R.; Snee, M.; Waring, M. J.; Wild, C.; London, N.; von Delft, F.; Walsh, M. A. Crystallographic and Electrophilic Fragment Screening of the SARS-CoV-2 Main Protease. Nat. Commun. 2020, 11, 5047. f) Craven, G. B.; Affron, D. P.; Kösel, T.; Wong, T. L. M.; Jukes, Z. H.; Liu, C.-T.; Morgan, R. M. L.; Armstrong, A.; Mann, D. J. Multiparameter Kinetic Analysis for Covalent Fragment Optimization Using Quantitative Irreversible Tethering (QIT). ChemBioChem 2020, 21, 3417-3422.

(18) Keeley, A.; Petri, L.; Ábrányi-Balogh, P.; Keserű, G. M. Covalent Fragment Libraries in Drug Discovery. Drug Discov. Today 2020, $25,983-996$.

(19) a) Hwang, K.-J.; Logusch, E. W. A Convenient Synthesis of Vinyl Sulfoximines from $\beta$-Hydroxyalkyl Sulfoximines. Tetrahedron Lett. 1987, 28, 4149-4152. b) Craig, D.; Geach, N. J. Synthesis of $E$ - $N$-Unfunctionalized $\alpha$, $\beta$-Unsaturated Sulfoximines and $N$-Trifluoromethanesulfonyl Derivatives. Synlett 1993, 7, 481-482.

(20) Erdelmeier, I.; Gais, H.-J. Stereoselective Synthesis of Enantiomerically Pure 1-(E)-Alkenylsulfoximines. Tetrahedron Lett. 1985, $26,4359-4362$.

(21) a) Hwang, K. J. A Convenient Synthesis of Free Vinyl Sulfoximines from Methyl Sulfoximines. Bull. Korean Chem. Soc. 2000, 21, 125-127. b) McGrath, M. J.; Bolm, C. The Use of Chiral Lithium Amides in the Desymmetrisation of $N$-Trialkylsilyl Dimethyl Sulfoximines. Beilstein J. Org. Chem. 2007, 3 (33). c) Kahraman, M.; Sinishtaj, S.; Dolan, P. M.; Kensler, T. W.; Peleg, S.; Saha, U.; Chuang, S. S.; Bernstein, G.; Korczak, B.; Posner, G. H. Potent, Selective and Low-Calcemic Inhibitors of CYP24 Hydroxylase: 24-Sulfoximine Analogues of the Hormone 1 $\alpha$,25-Dihydroxyvitamin D 3. J. Med. Chem. 2004, 47, 6854-6863. d) Gao, X.; Gaddam, V.; Altenhofer, E.; Tata, R. R.; Cai, Z.; Yongpruksa, N.; Garimallaprabhakaran, A. K.; Harmata, M. C-H Activation in S-Alkenyl Sulfoximines: An Endo 1,5-Hydrogen Migration. Angew. Chem. Int. Ed. 2012, 51, 7016-7019.

(22) Chinthakindi, P. K.; Nandi, G. C.; Govender, T.; Kruger, H. G.; Naicker, T.; Arvidsson, P. I. An Efficient Protecting-Group-Free Synthesis of Vinylic Sulfoximines via Horner-Wadsworth-Emmons Reaction. Synlett 2016, 27, 1423-1427.

(23) Carlström, K. E.; Chinthakindi, P. K.; Espinosa, B.; Al Nimer, F.; Arnér, E. S. J.; Arvidsson, P. I.; Piehl, F.; Johansson, K. Characterization of More Selective Central Nervous System Nrf2-Activating Novel Vinyl Sulfoximine Compounds Compared to Dimethyl Fumarate. Neurotherapeutics 2020, 17, 1142-1152. 
(24) Bräse, S.; Mende, M.; Jobelius, H. H.; Scharf, H.-D. Hydrazoic Acid and Azides. In Ullmann's Encyclopedia of Industrial Chemistry; Wiley-VCH Verlag GmbH \& Co. KGaA: Weinheim, Germany, 2015; pp 1-11.

(25) Mendiola, J.; Rincón, J. A.; Mateos, C.; Soriano, J. F.; de Frutos, O.; Niemeier, J. K.; Davis, E. M. Preparation, Use, and Safety of $O$ Mesitylenesulfonylhydroxylamine. Org. Process Res. Dev. 2009, 13, 263-267.

(26) Davies, T. Q.; Tilby, M. J.; Ren, J.; Parker, N. A.; Skolc, D.; Hall, A.; Duarte, F.; Willis, M. C. Harnessing Sulfinyl Nitrenes: A Unified One-Pot Synthesis of Sulfoximines and Sulfonimidamides. J. Am. Chem. Soc. 2020, 142, 15445-15453.

(27) For reviews, see: a) Bizet, V.; Hendriks, C. M. M.; Bolm, C. Sulfur Imidations: Access to Sulfimides and Sulfoximines. Chem. Soc. Rev. 2015, 44, 3378-3390. b) Bull, J. A.; Degennaro, L.; Luisi, R. Straightforward Strategies for the Preparation of NH-Sulfoximines: A Serendipitous Story. Synlett 2017, 28, 2525-2538. c) Nandi, G. C.; Arvidsson, P. I. Sulfonimidamides: Synthesis and Applications in Preparative Organic Chemistry. Adv. Synth. Catal. 2018, 360, 2976-3001. d) Wojaczyńska, E.; Wojaczyński, J. Modern Stereoselective Synthesis of Chiral Sulfinyl Compounds. Chem. Rev. 2020, 120, 4578-4611.

(28) For selected leading methods for sulfoximine synthesis, see: a) Okamura, H.; Bolm, C. Rhodium-Catalyzed Imination of Sulfoxides and Sulfides: Efficient Preparation of N-Unsubstituted Sulfoximines and Sulfilimines. Org. Lett. 2004, 6, 1305-1307. b) Lai, C.; Mathieu, G.; Gabrielli Tabarez, L. P.; Lebel, H. Batch and Continuous-Flow Iron(II)-Catalyzed Synthesis of Sulfilimines and Sulfoximines Using N -Mesyloxycarbamates. Chem. Eur. J. 2019, 25, 9423-9426. c) Zenzola, M.; Doran, R.; Luisi, R.; Bull, J. A. Synthesis of Sulfoximine Carbamates by Rhodium-Catalyzed Nitrene Transfer of Carbamates to Sulfoxides. J. Org. Chem. 2015, 80, 6391-6399. d) Mendonça Matos, P.; Lewis, W.; Argent, S. P.; Moore, J. C.; Stockman, R. A. General Method for the Asymmetric Synthesis of N-H Sulfoximines via C-S Bond Formation. Org. Lett. 2020, 22, 2776-2780. e) Aota, Y.; Kano, T.; Maruoka, K. Asymmetric Synthesis of Chiral Sulfoximines via the S-Arylation of Sulfinamides. J. Am. Chem. Soc. 2019, 141, 19263-19268. Also see references 30 and 31.

(29) For selected leading methods for sulfonimidamide synthesis, see: a) Yu, H.; Li, Z.; Bolm, C. Copper-Catalyzed Transsulfinamidation of Sulfinamides as a Key Step in the Preparation of Sulfonamides and Sulfonimidamides. Angew. Chem. Int. Ed. 2018, 57, 1560215605. b) Gao, B.; Li, S.; Wu, P.; Moses, J. E.; Sharpless, K. B. SuFEx Chemistry of Thionyl Tetrafluoride (SOF4) with Organolithium Nucleophiles: Synthesis of Sulfonimidoyl Fluorides, Sulfoximines, Sulfonimidamides, and Sulfonimidates. Angew. Chem. Int. Ed. 2018, 57, 19391943. c) Zasukha, S. V.; Timoshenko, V. M.; Tolmachev, A. A.; Pivnytska, V. O.; Gavrylenko, O.; Zhersh, S.; Shermolovich, Y.; Grygorenko, O. O. Sulfonimidamides and Imidosulfuric Diamides: Compounds from an Underexplored Part of Biologically Relevant Chemical Space. Chem. Eur. J. 2019, 25, 6928-6940. d) Bremerich, M.; Conrads, C. M.; Langletz, T.; Bolm, C. Additions to N-Sulfinylamines as an Approach for the Metal-Free Synthesis of Sulfonimidamides: O-Benzotriazolyl Sulfonimidates as Activated Intermediates. Angew. Chem. Int. Ed. 2019, 58, 19014-19020. e) Davies, T. Q.; Hall, A.; Willis, M. C. One-Pot, Three-Component Sulfonimidamide Synthesis Exploiting the Sulfinylamine Reagent N -Sulfinyltritylamine, TrNSO. Angew. Chem. Int. Ed. 2017, 56, 14937-14941. f) Greed, S.; Briggs, E. L.; Idiris, F. I. M.; White, A. J. P.; Lücking, U.; Bull, J. A. Synthesis of Highly Enantioenriched Sulfonimidoyl Fluorides and Sulfonimidamides by Stereospecific Sulfur-Fluorine Exchange (SuFEx) Reaction. Chem. Eur. J. 2020, 26, 12533-12538. Also See references 32 and 33.

(30) Zenzola, M.; Doran, R.; Degennaro, L.; Luisi, R.; Bull, J. A. Transfer of Electrophilic NH Using Convenient Sources of Ammonia: Direct Synthesis of NH Sulfoximines from Sulfoxides. Angew. Chem. Int. Ed. 2016, 55, 7203-7207.

(31) a) Tota, A.; Zenzola, M.; Chawner, S. J.; John-Campbell, S. S.; Carlucci, C.; Romanazzi, G.; Degennaro, L.; Bull, J. A.; Luisi, R. Synthesis of NH-Sulfoximines from Sulfides by Chemoselective One-Pot N- and O-Transfers. Chem. Commun. 2017, 53, 348-351. Also see: b) Lohier, J.-F.; Glachet, T.; Marzag, H.; Gaumont, A.-C.; Reboul, V. Mechanistic Investigation of the NH-Sulfoximination of Sulfide. Evidence for $\lambda 6$ Sulfanenitrile Intermediates. Chem. Commun. 2017, 53, 2064-2067. c) Chaabouni, S.; Lohier, J.-F.; Barthelemy, A.-L.; Glachet, T.; Anselmi, E.; Dagousset, G.; Diter, P.; Pégot, B.; Magnier, E.; Reboul, V. One-Pot Synthesis of Aryl- and Alkyl S -Perfluoroalkylated NH -Sulfoximines from Sulfides. Chem. Eur. J. 2018, 24, 17006-17010. d) Xie, Y.; Zhou, B.; Zhou, S.; Zhou, S.; Wei, W.; Liu, J.; Zhan, Y.; Cheng, D.; Chen, M.; Li, Y.; et al. Sulfimine-Promoted Fast 0 Transfer: One-Step Synthesis of Sulfoximine from Sulfide. ChemistrySelect 2017, 2, 1620-1624. e) Degennaro, L.; Tota, A.; De Angelis, S.; Andresini, M.; Cardellicchio, C.; Capozzi, M. A.; Romanazzi, G.; Luisi, R. Eur. J. Org. Chem. 2017, 44, 6486-6490. f) Tota, A.; Carlucci, C.; Pisano, L.; Cutolo, G.; Clarkson, G. J.; Romanazzi, G.; Degennaro, L.; Bull, J. A.; Rollin, P.; Luisi, R. Synthesis of Glycosyl Sulfoximines by a Highly Chemo- and Stereoselective NH- and O-Transfer to Thioglycosides. Org. Biomol. Chem. 2020, 18, 3893-3897.

(32) Izzo, F.; Schäfer, M.; Stockman, R.; Lücking, U. A New, Practical One-Pot Synthesis of Unprotected Sulfonimidamides by Transfer of Electrophilic NH to Sulfinamides. Chem. Eur. J. 2017, 23, 15189-15193.

(33) Briggs, E. L.; Tota, A.; Colella, M.; Degennaro, L.; Luisi, R.; Bull, J. A. Synthesis of Sulfonimidamides from Sulfenamides via an Alkoxy-Amino- $\lambda 6$-Sulfanenitrile Intermediate. Angew. Chem. Int. Ed. 2019, 58, 14303-14310.

(34) Worch, C.; Mayer, A. C.; Bolm, C. Synthesis and Use of Chiral Sulfoximines. In Organosulfur Chemistry in Asymmetric Synthesis; Wiley-VCH Verlag GmbH \& Co. KGaA: Weinheim, Germany; pp 209-232.

(35) Han, J.; Soloshonok, V. A.; Klika, K. D.; Drabowicz, J.; Wzorek, A. Chiral Sulfoxides: Advances in Asymmetric Synthesis and Problems with the Accurate Determination of the Stereochemical Outcome. Chem. Soc. Rev. 2018, 47, 1307-1350.

(36) Kuniyasu, H.; Ogawa, A.; Sato, K.; Ryu, I.; Kambe, N.; Sonoda, N. The First Example of Transition-Metal-Catalyzed Addition of Aromatic Thiols to Acetylenes. J. Am. Chem. Soc. 1992, 114, 5902-5903.

(37) Cao, C.; Fraser, L. R.; Love, J. A. Rhodium-Catalyzed Alkyne Hydrothiolation with Aromatic and Aliphatic Thiols. J. Am. Chem. Soc. 2005, 127, 17614-17615.

(38) Ogawa, A.; Ikeda, T.; Kimura, K.; Hirao, T. Highly Regio- and Stereocontrolled Synthesis of Vinyl Sulfides via Transition-MetalCatalyzed Hydrothiolation of Alkynes with Thiols. J. Am. Chem. Soc. 1999, 121, 5108-5114.

(39) Gerber, R.; Frech, C. M. Alkyne Hydrothiolation Catalyzed by a Dichlorobis(Aminophosphine) Complex of Palladium: Selective Formation of Cis-Configured Vinyl Thioethers. Chem. Eur. J. 2012, 18, 8901-8905.

(40) Shoai, S.; Bichler, P.; Kang, B.; Buckley, H.; Love, J. A. Catalytic Alkyne Hydrothiolation with Alkanethiols Using Wilkinson's Catalyst. Organometallics 2007, 26, 5778-5781.

(41) a) Kolb, H. C.; Sharpless, K. B. The Growing Impact of Click Chemistry on Drug Discovery. Drug Discov. Today 2003, 8, 1128-1137. b) Thirumurugan, P.; Matosiuk, D.; Jozwiak, K. Click Chemistry for Drug Development and Diverse Chemical-Biology Applications. Chem. Rev. 2013, 113, 4905-4979. c) Horisawa, K. Specific and Quantitative Labeling of Biomolecules Using Click Chemistry. Front. Physiol. 2014, $5,457$.

(42) a) Wright, M. H.; Sieber, S. A. Chemical Proteomics Approaches for Identifying the Cellular Targets of Natural Products. Nat. Prod. Rep. 2016, 33, 681-708. b) Van Kersavond, T.; Nguyen, M. T. N.; Verhelst, S. H. L. Synthesis and Application of Activity-Based Probes for Proteases. Methods in Molecular Biology; Humana Press Inc., 2017; Vol. 1574, pp 255-266. c) Pickens, C. J.; Johnson, S. N.; Pressnall, M. M.; Leon, M. A.; Berkland, C. J. Practical Considerations, Challenges, and Limitations of Bioconjugation via Azide-Alkyne Cycloaddition. Bioconjug. Chem. 2018, 29, 686-701. 
(43) a) Teng, F.; Sun, S.; Jiang, Y.; Yu, J.-T.; Cheng, J. Copper-Catalyzed Oxidative C(Sp3)-H/N-H Coupling of Sulfoximines and Amides with Simple Alkanes via a Radical Process. Chem. Commun. 2015, 51, 5902-5905. b) Zhu, H.; Teng, F.; Pan, C.; Cheng, J.; Yu, J.-T. Radical NArylation/Alkylation of Sulfoximines. Tetrahedron Lett. 2016, 57, 2372-2374. c) Bohnen, C.; Bolm, C. N-Trifluoromethylthiolated Sulfoximines. Org. Lett. 2015, 17, 3011-3013. d) Teng, F.; Cheng, J.; Yu, J.-T. Copper-Catalyzed N-Methylation/Ethylation of Sulfoximines. Org. Biomol. Chem. 2015, 13, 9934-9937. e) Kim, J.; Ok, J.; Kim, S.; Choi, W.; Lee, P. H. Mild Copper-TBAF-Catalyzed N -Arylation of Sulfoximines with Aryl Siloxanes. Org. Lett. 2014, 16, 4602-4605. f) Aithagani, S. K.; Dara, S.; Munagala, G.; Aruri, H.; Yadav, M.; Sharma, S.; Vishwakarma, R. A.; Singh, P. P. Metal-Free Approach for the Synthesis of N -Aryl Sulfoximines via Aryne Intermediate. Org. Lett. 2015, 17, 5547-5549. g) Zou, Y.; Xiao, J.; Peng, Z.; Dong, W.; An, D. Transition Metal-Free Aroylation of NH-Sulfoximines with Methyl Arenes. Chem. Commun. 2015, 51, 14889-14892. h) Zhao, Z.; Wang, T.; Yuan, L.; Jia, X.; Zhao, J. Oxidative Acylation of Sulfoximines with Methylarenes as an Acyl Donor. RSC Adv. 2015, 5, 75386-75389. i) Bala, B. D.; Sharma, N.; Sekar, G. Sulfoximinocarbonylation of Aryl Halides Using Heterogeneous Pd/C Catalyst. RSC Adv. 2016, 6, 97152-97159.

(44) Izzo, F.; Schäfer, M.; Lienau, P.; Ganzer, U.; Stockman, R.; Lücking, U. Exploration of Novel Chemical Space: Synthesis and in Vitro Evaluation of N-Functionalized Tertiary Sulfonimidamides. Chem. Eur. J. 2018, 24, 9295-9304.

(45) Mabasa, T. F.; Awe, B.; Laming, D.; Kinfe, H. H. Design, Synthesis and Antiplasmodial Evaluation of Sulfoximine-Triazole Hybrids as Potential Antimalarial Prototypes. Med. Chem. 2019, 15, 685-692.

(46) a) Gegenava, M.; Chankvetadze, L.; Farkas, T.; Chankvetadze, B. Enantioseparation of Selected Chiral Sulfoxides in High-Performance Liquid Chromatography with Polysaccharide-Based Chiral Selectors in Polar Organic Mobile Phases with Emphasis on Enantiomer Elution Order. J. Sep. Sci. 2014, 37, 1083-1088. b) Sardella, R.; Ianni, F.; Di Michele, A.; Di Capua, A.; Carotti, A.; Anzini, M.; Natalini, B. Enantioresolution and Stereochemical Characterization of Two Chiral Sulfoxides Endowed with COX-2 Inhibitory Activity. Chirality 2017, 29, 536-540. c) Ferretti, R.; Carradori, S.; Guglielmi, P.; Pierini, M.; Casulli, A.; Cirilli, R. Enantiomers of Triclabendazole Sulfoxide: Analytical and Semipreparative HPLC Separation, Absolute Configuration Assignment, and Transformation into Sodium Salt. J. Pharm. Biomed. Anal. 2017, 140, 38-44.

(47) a) del Nozal, M. J.; Toribio, L.; Bernal, J. L.; Alonso, C.; Jiménez, J. J. Chiral Separation of Omeprazole and Several Related Benzimidazoles Using Supercritical Fluid Chromatography. J. Sep. Sci. 2004, 27, 1023-1029. b) West, C.; Konjaria, M.-L.; Shashviashvili, N.; Lemasson, E.; Bonnet, P.; Kakava, R.; Volonterio, A.; Chankvetadze, B. Enantioseparation of Novel Chiral Sulfoxides on Chlorinated Polysaccharide Stationary Phases in Supercritical Fluid Chromatography. J. Chromatogr. A 2017, 1499, 174-182.

(48) Parsons, S. Determination of Absolute Configuration Using X-Ray Diffraction. Tetrahedron: Asymmetry 2017, 28, 1304-1313.

(49) Seco, J. M.; Quiñoá, E.; Riguera, R. The Assignment of Absolute Configuration by NMR. Chem. Rev. 2004, 1, 17-117.

(50) Polavarapu, P. Determination of the Absolute Configurations of Chiral Drugs Using Chiroptical Spectroscopy. Molecules 2016, 21, 1056.

(51) Berova, N.; Bari, L. Di; Pescitelli, G. Application of Electronic Circular Dichroism in Configurational and Conformational Analysis of Organic Compounds. Chem. Soc. Rev. 2007, 36, 914.

(52) Xiong, F.; Yang, B.-B.; Zhang, J.; Li, L. Enantioseparation, Stereochemical Assignment and Chiral Recognition Mechanism of Sulfoxide-Containing Drugs. Molecules 2018, 23, 2680.

(53) Worch, C.; Atodiresei, I.; Raabe, G.; Bolm, C. Synthesis of Enantiopure Sulfonimidamides and Elucidation of Their Absolute Configuration by Comparison of Measured and Calculated CD Spectra and X-Ray Crystal Structure Determination. Chem. Eur. J. 2010, 16, 677683.

(54) Bruhn, T.; Schaumlöffel, A.; Hemberger, Y.; Bringmann, G. SpecDis: Quantifying the Comparison of Calculated and Experimental Electronic Circular Dichroism Spectra. Chirality 2013, 25, 243-249.

(55) https://reachseparations.com/

(56) Wimmer, A.; König, B. N -Arylation of NH -Sulfoximines via Dual Nickel Photocatalysis. Org. Lett. 2019, 21, 2740-2744.

(57) Bach, T.; Körber, C. The Preparation of N-Tert-Butyloxycarbonyl-(Boc-)Protected Sulfoximines and Sulfimines by an Iron(II)-

Mediated Nitrene Transfer from BocN3 to Sulfoxides and Sulfides. Eur. J. Org. Chem. 1999, 1999, 1033-1039.

(58) Dong, S.; Frings, M.; Cheng, H.; Wen, J.; Zhang, D.; Raabe, G.; Bolm, C. Organocatalytic Kinetic Resolution of Sulfoximines. J. Am. Chem. Soc. 2016, 138, 2166-2169.

(59) Kuniyasu, H.; Ogawa, A.; Sato, K.; Ryu, I.; Kambe, N.; Sonoda, N. The First Example of Transition-Metal-Catalyzed Addition of Aromatic Thiols to Acetylenes. J. Am. Chem. Soc. 1992, 114, 5902-5903.

(60) Zheng, Y.; Du, X.; Bao, W. L-Proline Promoted Cross-Coupling of Vinyl Bromide with Thiols Catalyzed by CuBr in Ionic Liquid. Tetrahedron Lett. 2006, 47, 1217-1220.

(61) Leca, D.; Song, K.; Amatore, M.; Fensterbank, L.; Lacôte, E.; Malacria, M. Iodine(III)-Mediated Preparations of Nitrogen-Containing Sulfur Derivatives: Dramatic Influence of the Sulfur Oxidation State. Chem. Eur. J. 2004, 10, 906-916.

(62) Gaussian 16, Revision C.01, M. J. Frisch, G. W. Trucks, H. B. Schlegel, G. E. Scuseria, M. A. Robb, J. R. Cheeseman, G. Scalmani, V. Barone, G. A. Petersson, H. Nakatsuji, X. Li, M. Caricato, A. V. Marenich, J. Bloino, B. G. Janesko, R. Gomperts, B. Mennucci, H. P. Hratchian, J. V. Ortiz, A. F. Izmaylov, J. L. Sonnenberg, D. Williams-Young, F. Ding, F. Lipparini, F. Egidi, J. Goings, B. Peng, A. Petrone, T. Henderson, D. Ranasinghe, V. G. Zakrzewski, J. Gao, N. Rega, G. Zheng, W. Liang, M. Hada, M. Ehara, K. Toyota, R. Fukuda, J. Hasegawa, M. Ishida, T. Nakajima, Y. Honda, O. Kitao, H. Nakai, T. Vreven, K. Throssell, J. A. Montgomery, Jr., J. E. Peralta, F. Ogliaro, M. J. Bearpark, J. J. Heyd, E. N. Brothers, K. N. Kudin, V. N. Staroverov, T. A. Keith, R. Kobayashi, J. Normand, K. Raghavachari, A. P. Rendell, J. C. Burant, S. S. Iyengar, J. Tomasi, M. Cossi, J. M. Millam, M. Klene, C. Adamo, R. Cammi, J. W. Ochterski, R. L. Martin, K. Morokuma, O. Farkas, J. B. Foresman, and D. J. Fox, Gaussian, Inc., Wallingford CT, 2019. 
